\title{
Stability of Quantum Systems at Three Scales: Passivity, Quantum Weak Energy Inequalities and the Microlocal Spectrum Condition
}

\author{
Christopher J. Fewster ${ }^{1}$ and Rainer $\mathrm{VerCH}^{2, *}$ \\ ${ }^{1}$ Department of Mathematics, \\ University of York, \\ Heslington, \\ York YO10 5DD, United Kingdom \\ e-mail: cjf3@york.ac.uk \\ ${ }^{2}$ Institut für Theoretische Physik, \\ Universität Göttingen, \\ Bunsenstr. 9, \\ D-37073 Göttingen, Germany \\ revised - 3 April 2003
}

\begin{abstract}
Quantum weak energy inequalities have recently been extensively discussed as a condition on the dynamical stability of quantum field states, particularly on curved spacetimes. We formulate the notion of a quantum weak energy inequality for general dynamical systems on static background spacetimes and establish a connection between quantum weak energy inequalities and thermodynamics. Namely, for such a dynamical system, we show that the existence of a class of states satisfying a quantum weak inequality implies that passive states (e.g., mixtures of ground- and thermal equilibrium states) exist for the time-evolution of the system and, therefore, that the second law of thermodynamics holds. As a model system, we consider the free scalar quantum field on a static spacetime. Although the Weyl algebra does not satisfy our general assumptions, our abstract results do apply to a related algebra which we construct, following a general method which we carefully describe, in Hilbert-space representations induced by quasifree Hadamard states. We discuss the problem of reconstructing states on the Weyl algebra from states on the new algebra and give conditions under which this may be accomplished.

Previous results for linear quantum fields show that, on one hand, quantum weak energy inequalities follow from the Hadamard condition (or microlocal spectrum condition) imposed on the states, and on the other hand, that the existence of passive states implies that there is a class of states fulfilling the microlocal spectrum condition. Thus, the results of this paper indicate that these three conditions of dynamical stability are essentially equivalent. This observation is significant because the three conditions become effective at different length scales: The microlocal spectrum condition constrains the short-distance behaviour of quantum states (microscopic stability), quantum weak energy inequalities impose conditions at finite distance (mesoscopic stability), and the existence of passive states is a statement on the global thermodynamic stability of the system (macroscopic stability).
\end{abstract}

\footnotetext{
${ }^{*}$ Current address: Max-Planck-Institute for Mathematics in the Sciences, Inselstr. 22, 04103 Leipzig, Germany. Email: verch@mis.mpg.de
} 


\section{Introduction}

At small scales and high energies, the behaviour of matter is governed by quantum field theory, and it is widely known that the energy-momentum tensor in quantum field theory violates the energy-positivity conditions that are generically fulfilled for classical matter in general relativity. For instance, in flat spacetime, there is in any quantum field theory a large class of physical states for which the expectation value of the energy density is a function on spacetime assuming negative as well as positive values [10. However, as pointed out by Ford [17, one may argue on heuristic grounds that there must be constraints on the intensity and spatio-temporal extension of negative values of the energy density, as otherwise one could produce situations in which macroscopic violations of the second law of thermodynamics occur. This idea was further developed by Ford and several other authors (see, e.g., [18, 19, 32, 12, 11, 13, 14, 16, 47, 15, 30]) and led to a form of such constraints which are now called quantum weak energy inequalities (abbreviated, QWEIs) in the terminology of [15], or often simply quantum inequalities (QIs). To explain their nature, consider some quantum field propagating on a Lorentzian spacetime $(M, \boldsymbol{g})$,

and let $\left\langle T_{\mu \nu}(x)\right\rangle_{\omega}$ be the expectation value of the energy-momentum tensor in a state (expectation functional) $\omega$ at some spacetime point $x$. For any smooth, timelike curve $\gamma$ in spacetime, denote by

$$
\rho_{\omega}(\tau)=\dot{\gamma}^{\mu}(\tau) \dot{\gamma}^{\nu}(\tau)\left\langle T_{\mu \nu}(\gamma(\tau))\right\rangle_{\omega}
$$

the mean energy density in the state $\omega$ along the curve, parametrized by proper time $\tau$. Then one says that the states of the quantum field fulfill a QWEI if for any timelike curve $\gamma$ and any real-valued smooth, compactly supported test-function $g$ there holds a bound of the form

$$
\inf _{\omega} \int d \tau g^{2}(\tau) \rho_{\omega}(\tau) \geq c_{g, \gamma}>-\infty
$$

where the infimum is taken over the set of states $\omega$ of the quantum field theory in which $\rho_{\omega}$ can be reasonably defined as a locally integrable function (typically, this is a dense set of all physical states for the quantum field theory). The important point is that the constant $c_{g, \gamma}$ bounding the weighted integral of the energy density along $\gamma$ from below may depend on the weight-function $g$ and the curve $\gamma$, but is state-independent.

To comment on this constraint, we note first that an inequality of the form (1.1) has been shown to hold for the class of Hadamard states $\omega$ of the free Klein-Gordon field and the Dirac field on arbitrary, globally hyperbolic spacetimes [12, 15. Moreover, in more special situations, the inequality (1.1) was obtained in a more specific form. For example, for the massless free scalar field in $d$-dimensional Minkowski spacetime and the specific choice of a (not compactly supported) Lorentzian weight function $g^{2}(\tau)=t_{0} /\left[\pi\left(\tau^{2}+t_{0}^{2}\right)\right]$ $\left(t_{0}>0\right)$, and taking for $\gamma$ any straight timelike line, one obtains a bound

$$
\frac{t_{0}}{\pi} \int d \tau \frac{\rho_{\omega}(\tau)}{\tau^{2}+t_{0}^{2}} \geq-\frac{\Gamma_{d}}{t_{0}^{d}}
$$

for all states $\omega$ of finite particle number and energy, where $\Gamma_{d}$ is a state-independent universal constant depending only on the spacetime dimension [19, 16, 11]. Thus the 
intensity of the weighted negative energy is at most proportional to an inverse power of its mean duration, i.e. the width $t_{0}$ of the Lorentzian weight function. This considerably limits the possibility of negative energies to build up to macroscopic violations of the second law of thermodynamics. Also, other macroscopic dynamical instabilities are hampered in this way when taking expectation values of a quantum field's energy-momentum tensor as the right hand side of Einstein's equations of gravity: It can be shown that appropriate forms of averaged energy conditions such as (1.1) or (1.2) imply similar statements about the dynamical behaviour of solutions to Einstein's equations as do the pointwise energy positivity conditions for classical matter. Examples include singularity theorems, or the impossiblity of exotic spacetimes with closed timelike curves or "warp-drive" scenarios 46, 20, 31.

The argument sketched above - that a bound on negative energy densities in quantum field theory of the type (1.2) really prevents a macroscopic violation of the second law of thermodynamics - is, of course, somewhat heuristic. The purpose of the present article is to show that such a conclusion may be drawn rigorously under fairly general circumstances. To this end, we shall specialize our setting a bit and consider a quantum system (which may, but need not, be a quantum field) situated in a static, spatially compact spacetime (the assumption of spatial compactness is mainly made for convenience). Thus, the underlying spacetime $(M, \boldsymbol{g})$ is of the form

$$
M=\mathbb{R} \times \Sigma,
$$

where $\Sigma$ is a smooth, $s$-dimensional compact manifold endowed with a Riemannian metric $\boldsymbol{h}$, and the static Lorentzian metric $\boldsymbol{g}$ on $M$ has the line element

$$
d s^{2}=g_{a b} d x^{a} d x^{b}=g_{00} d t^{2}-h_{i j} d x^{i} d x^{j}
$$

with a smooth, strictly positive function $g_{00}$ on $\Sigma$. Consequently, when a point $x \in M$ is represented as $x=(t, \underline{x})$ with $t \in \mathbb{R}$ and $\underline{x} \in \Sigma$, then $\boldsymbol{g}$ is independent of $t$. The variable $t$ is to be viewed as "time"-variable, whereas $\Sigma$ contains all the spatial locations of the system at instances of "equal time".

We will then assume that the quantum system on this spacetime can be modelled such that the observables of the system are elements of a $C^{*}$-algebra ${ }^{1} \mathcal{A}$, and that the time-evolution of the system can be described by a one-parameter group $\left\{\alpha_{t}\right\}_{t \in \mathbb{R}}$ of automorphisms of $\mathcal{A}$. A technical assumption is also made, namely that the automorphism group is strongly continuous in time: This means that $\left\|\alpha_{t}(A)-A\right\| \rightarrow 0$ as $t \rightarrow 0$ for each $A \in \mathcal{A}$; the norm appearing here is the $C^{*}$-norm on $\mathcal{A}$. In technical terms (cf. [6]), the pair $\left(\mathcal{A},\left\{\alpha_{t}\right\}_{t \in \mathbb{R}}\right)$ is a $C^{*}$-dynamical system. Here the time-parameter $t$ is thought of as having the significance of the time-parameter of the spacetime, so that $\left\{\alpha_{t}\right\}_{t \in \mathbb{R}}$ describes the time-evolution of the system on the underlying spacetime. That is, if the system is formed by a family $\mathcal{A}(O) \subset \mathcal{A}, O \subset \mathbb{R} \times \Sigma$, of sub- $C^{*}$-algebras of $\mathcal{A}$ fulfilling the isotony condition $O_{1} \subset O \Rightarrow \mathcal{A}\left(O_{1}\right) \subset \mathcal{A}(O)$, then we assume that $\alpha_{t}(\mathcal{A}(O))=\mathcal{A}\left(\tau_{t}(O)\right)$ where $\tau_{t}$ denotes the time-shift $\tau_{t}\left(t^{\prime}, \underline{x}\right)=\left(t+t^{\prime}, \underline{x}\right)$ on the underlying spacetime. These assumptions are very general (cf. [23]); apart from some points of technical detail (such as

\footnotetext{
${ }^{1}$ We shall understand by a $C^{*}$-algebra always a $C^{*}$-algebra containing a unit element, generically denoted by 1 .
} 
replacing the $C^{*}$-algebra by a more general types of $*$-algebra, or relaxing the assumption of strong continuity of the dynamics), there is a vast range of quantum systems on static spacetimes that can be modelled as $C^{*}$-dynamical systems.

Some comments on the extent to which the assumption of strong countinuity is realized in quantum field theoretic system may nevertheless be helpful. The typical description of such systems in the present setting of a static spacetime $M$ starts with a $C^{*}$-algebra $\mathfrak{A}$ generated by elements $\mathrm{G}(f)$, where $f$ ranges over $C_{0}^{\infty}(M)$. These algebraic generators are subject to certain relations characterizing dynamical properties of the system, and a time evolution can usually be defined on $\mathfrak{A}$ as a one-parametric group $\left\{\tilde{\alpha}_{t}\right\}_{t \in \mathbb{R}}$ of automorphisms of $\mathfrak{A}$ via $\tilde{\alpha}_{t}(\mathrm{G}(f))=\mathrm{G}\left(f \circ \tau_{-t}\right)$. However, thinking in particular of the case of a free bosonic field, where $\mathbf{G}(f)$ are the Weyl-generators of a CCR-algebra ${ }^{2}$, the corresponding $\left\{\tilde{\alpha}_{t}\right\}_{t \in \mathbb{R}}$ will, in general, fail to be strongly continuous. This difficulty can be overcome by passing to sufficiently "regular" Hilbert-space representations $\pi$ of $\mathfrak{A}$ in which $\left\{\tilde{\alpha}_{t}\right\}_{t \in \mathbb{R}}$ is weakly continuous. We will give a very detailed account of this construction for the example of the scalar Klein-Gordon field in Sec. 4, to prepare the ground for this, we now outline the basic idea.

To simplify the discussion a little bit, and to help the reader who is not too familiar with $C^{*}$-dynamical systems to see the connection with the more common Hilbert space approach, we assume that there is a weakly continuous unitary group $\left\{V_{t}\right\}_{t \in \mathbb{R}}$ on $\mathcal{H}$, the representation Hilbert space of $\pi$, so that

$$
V_{t} \pi(\mathrm{A}) V_{t}^{*}=\pi\left(\tilde{\alpha}_{t}(\mathrm{~A})\right)
$$

for all $\mathrm{A} \in \mathfrak{A}$. Then $\alpha_{t}(A)=V_{t} A V_{t}^{*}, A \in \pi(\mathfrak{A})$, defines a group of automorphisms of $\pi(\mathfrak{A}) \subset B(\mathcal{H})$ which need not be strongly strongly continuous but only weakly continuous, i.e. $\left\langle\psi,\left(\alpha_{t}(B)-B\right) \phi\right\rangle \rightarrow 0$ as $t \rightarrow 0$ for all choices of vectors $\phi, \psi \in \mathcal{H}$. But this form of continuity implies that for each $h \in C_{0}^{\infty}(\mathbb{R})$ and each $A \in \pi(\mathfrak{A})$ the weak (or Bochner-) integral

$$
\alpha_{h} A=\int d t h(t) \alpha_{t}(A)
$$

exists as an element in $B(\mathcal{H})$; more precisely, $\alpha_{h} A$ is contained in $\pi(\mathfrak{A})^{\prime \prime}$, the von Neumann algebra generated by $\pi(\mathfrak{A}){ }^{3} \quad$ Moreover, it is easy to see (cf. Sec. 4) that $\| \alpha_{t}\left(\alpha_{h} A\right)-$ $\alpha_{h} A \| \rightarrow 0$ for $t \rightarrow 0$. Hence, if we denote by $\mathcal{A}$ the $C^{*}$-algebra which is generated by all the $\alpha_{h} A$ where $A \in \pi(\mathfrak{A})$ and $h \in C_{0}^{\infty}(\mathbb{R})$, then $\left(\mathcal{A},\left\{\alpha_{t}\right\}_{t \in \mathbb{R}}\right)$ forms a $C^{*}$-dynamical system. (The local algebras $\mathcal{A}(O)$ are then defined as $\mathcal{A} \cap\left\{\pi(\mathrm{G}(f)): f \in C_{0}^{\infty}(O)\right\}^{\prime \prime}$.)

Therefore one sees that $C^{*}$-dynamical systems arise naturally as a means of describing quantum field systems as soon as one considers representations in which the time-evolution of the system is modelled by a weakly continuous unitary group (and also under more general circumstances, see Sec. 4), and this is surely a very general situation, irrespective of whether a quantum field is interacting or free. We should also emphasize that, once such a representation $\pi$ is chosen, it doesn't matter if one describes the system in terms of the algebra $\pi(\mathfrak{A})$ or the algebra $\mathcal{A}$ since, for each unit vector $\psi \in \mathcal{H}$, the expectation value $\langle\psi, A \psi\rangle$ of $A \in \pi(\mathfrak{A})$ can be approximated as closely as desired by the expectation value

\footnotetext{
${ }^{2}$ See Sec. 4.1 for a fuller discussion of the CCR-algebra in the context of the free Klein-Gordon field.

${ }^{3}$ For a subset $\mathcal{B}$ of $B(\mathcal{H}), \mathcal{B}^{\prime}$ denotes the commutant of $\mathcal{B}$, i.e. $\mathcal{B}^{\prime}=\{C \in B(\mathcal{H}): C B=B C \forall B \in \mathcal{B}\}$.
} 
$\left\langle\psi, \alpha_{h} A \psi\right\rangle$ of $\alpha_{h} A \in \mathcal{A}$ through sharply peaking $h$ around 0. Conversely, $\alpha_{h} A$ is weaky approximated by elements of $\pi(\mathfrak{A})$. For further discussion of the relation between states on $\mathcal{A}$ and states on $\mathfrak{A}$ in the case of the CCR-algebra, see towards the end of Sec. 4.1 and Appendix A.5.

To make contact with thermodynamics, we now turn to the notion of passivity introduced by Pusz and Woronowicz [33. Let $\left(\mathcal{A},\left\{\alpha_{t}\right\}_{t \in \mathbb{R}}\right)$ be a $C^{*}$-dynamical system. The idea of Pusz and Woronowicz was to consider the behaviour of the system when the external conditions change in time. To this end, we note first (cf. Sec. 22) that there exists a norm-dense $*$-subalgebra $D(\delta)$ of $\mathcal{A}$ so that

$$
\delta(A)=\left.\frac{d}{d t} \alpha_{t}(A)\right|_{t=0}, \quad A \in D(\delta),
$$

exists in $\mathcal{A}$. This derivation is the generator of the dynamics when the external conditions remain unchanged. A change in the external conditions can be modelled via replacing $\delta$ by a time-dependent dynamics-generator

$$
\delta_{t}(A)=\delta(A)+i\left[H_{t}, A\right], \quad A \in D(\delta),
$$

where $H_{t}=H_{t}^{*}$ is a smooth function of $t \in \mathbb{R}$ having values in $\mathcal{A}$. Now assume that $H_{t}=0$ for $t<0$ and for $t>T$ where $T$ is some positive number. Then the dynamics of the system remains unchanged before $t=0$ and after $t=T$. In other words, the system undergoes a cyclic change of external conditions during which it is thermally isolated. (For $t<0$ and $t>T$, the system is closed.) It can be shown that there is a unique smooth family $U_{t}^{H}, t \in \mathbb{R}$, of unitary elements in $\mathcal{A}$ solving the initial value problem

$$
\frac{d}{d t} U_{t}^{H}=\frac{1}{i} \alpha_{t}\left(H_{t}\right) U_{t}^{H}, \quad U_{0}^{H}=1
$$

and that, consequently, the family $\alpha_{t}^{H}, t \in \mathbb{R}$, of automorphisms of $\mathcal{A}$ given by $\alpha_{t}^{H}(A)=$ $U_{t}^{H *} \alpha_{t}(A) U_{t}^{H}$ solves the initial value problem

$$
\frac{d}{d t} \alpha_{t}^{H}(A)=\alpha_{t}^{H}\left(\delta_{t}(A)\right), \quad \alpha_{0}^{H}(A)=A,
$$

for all $A \in D(\delta)$.

We recall that a state on a $C^{*}$-algebra $\mathcal{A}$ is a continuous linear functional $\omega: \mathcal{A} \rightarrow \mathbb{C}$ which is positive, i.e. $\omega\left(A^{*} A\right) \geq 0$ for all $A \in \mathcal{A}$, and fulfills $\omega(\mathbf{1})=1$.

If the system is initially in the state $\omega$, then the work done on the system under the cyclic change of external conditions is

$$
L^{H}(\omega)=\int_{0}^{T} d t \omega\left(\alpha_{t}^{H}\left(\frac{d H_{t}}{d t}\right)\right),
$$

and, as shown in 33], it holds that

$$
L^{H}(\omega)=\frac{1}{i} \omega\left(U_{T}^{H *} \delta\left(U_{T}^{H}\right)\right) .
$$


Pusz and Woronowicz call a state $\omega$ on $\mathcal{A}$ passive if $L^{H}(\omega) \geq 0$ for all smooth $H: \mathbb{R} \ni$ $t \mapsto H_{t}=H_{t}^{*} \in \mathcal{A}$ that have $H_{t}=0$ for $t$ outside $[0, T]$ with some $T>0$. Then it follows from (1.7) that $\omega$ is passive if and only if

$$
\inf _{U \in \mathcal{U}_{0}(\delta)} \frac{1}{i} \omega\left(U^{*} \delta(U)\right) \geq 0
$$

where $\mathcal{U}_{0}(\delta)$ denotes the set of all unitary $U$ in $D(\delta)$ which are in $\mathcal{A}$ continuously connected to 1 .

Passive states may thus be viewed as states of the system which are "in equilibrium" in the sense that, if the system is in such a state, then it is impossible to extract energy (gain work) from the system under cyclic changes of the external conditions of the system (while keeping it thermally isolated). It is in this sense that the second law of thermodynamics is valid for passive states. However, the set of passive states is really larger than the class of thermal equilibrium states, as those have a definite temperature: Each mixture of thermal equilibrium states (KMS-states) at arbitrary temperatures (including zero, corresponding to ground states) is a passive state. On the other hand, passive states are invariant under $\left\{\alpha_{t}\right\}_{t \in \mathbb{R}}$, and some (in most circumstances, mild) additional conditions such as clustering, or complete passivity, imply that passive states are, in fact, thermal equilibrium states at a definite temperature, i.e. KMS-states or ground states. We refer to [33] for a detailed discussion of these matters. We should like to mention that, in the context of quantum field theory in Minkowski-spacetime, Kuckert [28] has recently introduced a weaker notion of passivity, called semipassivity, and has shown that for stationary and homogeneous states semipassivity is equivalent to the KMS-condition for a particular inertial observer. There are also more recently investigated interconnections between energy-compactness, semi-passivity, and thermal equilibrium properties of states in quantum field theory [29, 22, 1] to which we would like to direct the reader's attention.

In the present work, we shall show that the existence of passive states for $C^{*}$-dynamical systems modelled on a static, spatially compact spacetime can be deduced from a suitable form of a QWEI. For this purpose, we suppose that there exists an energy density whose spatial integral yields the generator of the dynamics, and we assume QWEIs for this energy-density to hold for a suitable dense set of states on $\mathcal{A}$. The precise assumptions will be discussed in Sec. 2. Given these assumptions, along with some other physically well-motivated conditions, we obtain in Sec. 2 several assertions about the existence of passive states for the $C^{*}$-dynamical system. While the investigations in Sec. 2 are based on general, model-independent assumptions, Sec. 4 concerns the example of the free scalar Klein-Gordon field on a static, spatially compact spacetime. In particular, we will show that in the GNS-representation of each quasifree Hadamard state one obtains a $C^{*}$-dynamical system and an energy density on a suitable domain such that all the general assumptions of Sec. 2 are satisfied. It is of course well-known that quasifree Hadamard ground- and KMS-states exist for the free scalar field on static, spatially compact spacetimes [9], but we emphasise that our arguments do not make use of this fact. Thus there is the prospect that the results of Sec. 2 have a wider applicability beyond the realm of free quantum fields. Instead, our arguments rely heavily on the characterization of the Hadamard property in terms of a condition on the wavefront set of the two-point function of a state for the free field, called microlocal spectrum condition, which was established in 
[34] (cf. also [3, 38]). However, we shall work with a new version of that characterization taken in part from [40] and related to the concept of "domain of microlocal smoothness" in [2]. Thus, Sec. [4 contains several apparently new microlocal techniques in quantum field theory on curved spacetime. Some background material on microlocal analysis, including a convenient calculus for distributions taking values in Banach and Hilbert spaces is presented in Sec. [3.

To conclude this introduction, we would like to point out that the results obtained in this work, together with results previously obtained, indicate an intimate connection and apart from points of technical detail, equivalence - between the following classes of states of a free quantum field (on static spacetimes):

(i) states fulfilling the microlocal spectrum condition (Hadamard states),

(ii) states fulfilling QWEIs,

(iii) states induced by operations on passive states (corresponding to local, finite energy excitations of passive states).

Namely, it was shown in [12] (see also [15]) that free quantum field states fulfilling the microlocal spectrum condition satify QWEIs. In the present work, we show that QWEIs imply the existence of passive states. On the other hand, 37] indicates that passive states fulfill the microlocal spectrum condition (and this result carries over to a suitable dense set of states in the GNS-representation of passive states, cf. [45]).

This observation is of considerable significance since the regularity properties on the classes of states (i), (ii) and (iii) are imposed on very different length scales. The states (i) fulfill a constraint on their microscopic short-distance behaviour, the condition on the states (ii) is imposed at finite length scales while the specification of the class of states (iii) is a global condition on their thermodynamic stability. We conjecture that this connection between dynamical stability conditions at different length scales is a generic feature and extends to physical systems described by more general types of quantum fields.

\section{Passivity from quantum weak energy inequalities}

We will consider a quantum system situated in a static spacetime of the form $\mathbb{R} \times \Sigma$, where $\Sigma$ is an $s$-dimensional compact manifold; the variable $t \in \mathbb{R}$ is interpreted as "time"-variable, whereas $\Sigma$ contains all spatial locations of the system. Furthermore, we assume that the spacetime is endowed with a static metric of the form (1.3). Then this metric induces a preferred measure on the Borel-sets of $\Sigma$ which we will denote by $d \mu$; it is the volume measure of the Riemannian metric $\boldsymbol{h}$ on $\Sigma$. In local coordinates $\left(\underline{x}^{i}\right)$ for $\Sigma, d \mu(\underline{x})$ has the coordinate expression $\sqrt{\operatorname{det}\left(h_{i j}\right)} d^{s} \underline{x}$. (We note that everywhere in the discussion to follow one could replace $d \mu$ by any other Borel-measure on $\Sigma$, but the choice just made is convenient and natural.)

We shall make some general, model-independent assumptions concerning the mathematical description of the dynamical properties of the system, suited to discuss the connection between QWEIs and passivity. In Section 4 we will show that all these assumptions are fulfilled in the case of the free scalar Klein-Gordon field on a static, globally 
hyperbolic spacetime.

Our quantum system will be modelled by a $C^{*}$-dynamical system $\left(\mathcal{A},\left\{\alpha_{t}\right\}_{t \in \mathbb{R}}\right)$ as discussed in the Introduction.

Since $\left\{\alpha_{t}\right\}_{t \in \mathbb{R}}$ is strongly continuous, there exists a norm-dense (maximal) domain $D(\delta)$ in $\mathcal{A}$ so that the derivation

$$
\delta(A)=\left.\frac{d}{d t} \alpha_{t}(A)\right|_{t=0}
$$

exists in $\mathcal{A}$ for all $A \in D(\delta)$. In fact, $D(\delta)$ is a norm-dense $*$-subalgebra of $\mathcal{A}$, as is the set $D^{\infty}(\delta)$ of all elements in $\mathcal{A}$ such that $t \mapsto \alpha_{t}(A)$ is $C^{\infty}$ at $t=0$ (i.e., the common domain for $\delta^{n}$, for all $n \in \mathbb{N}$ ), since defining for each $A \in \mathcal{A}$ and $h \in C_{0}^{\infty}(\mathbb{R})$ their convolution $\alpha_{h} A$ with respect to $\left\{\alpha_{t}\right\}_{t \in \mathbb{R}}$ by (1.4) (with the integral now converging in norm because of strong continuity) one finds that $\alpha_{h} A \in D^{\infty}(\delta)$.

Next, we need to introduce an energy-density in order to connect our dynamical system with QWEIs. Since energy-densities are in many examples unbounded operators or quadratic forms, we need an appropriate domain for such a quantity with convenient algebraic and density properties. Therefore, we shall now make the assumption that there is a norm-dense subspace $\mathcal{W}^{\infty} \subset D^{\infty}(\delta)$ which has the property of being stable under taking adjoints and under convolution with test-functions with respect to the action of $\left\{\alpha_{t}\right\}_{t \in \mathbb{R}}$. In other words, for all $A \in \mathcal{W}^{\infty}$ and $f \in C_{0}^{\infty}(\mathbb{R}), \alpha_{f} A$ is also contained in $\mathcal{W}^{\infty}$. We shall denote by $\mathcal{A}^{\infty}$ the *-algebra generated by $\mathcal{W}^{\infty}$.

It will turn out to be convenient to introduce the set $\mathcal{U}^{\infty} \subset D^{\infty}(\delta)$ of all unitaries $U$ which are of the form

$$
U=\mathrm{e}^{i A_{1}} \cdots \mathrm{e}^{i A_{N}}
$$

where $N \in \mathbb{N}$ and $A_{1}, \ldots, A_{N}$ are hermitean elements in $\mathcal{A}^{\infty}$. Lemma 2.2 below will show that it suffices to consider $U$ of this form when discussing general cyclic changes to the system such as that described by $U_{t}^{H}$ in Eqs. (1.6) and (1.7). We denote by $\mathcal{U}_{\text {alg }}^{\infty}$ the *algebra which $\mathcal{U}^{\infty}$ generates; this is a sub-*-algebra of $D^{\infty}(\delta)$. (In fact, as a consequence of Lemma 2.2 below, the closures of $\mathcal{U}_{\mathrm{alg}}^{\infty}$ and $D^{\infty}(\delta)$ in the graph-norm of $\delta$ coincide.)

Now we can formulate our assumptions on the existence of an energy density generating the dynamics: We assume that there exists a set $\mathcal{S}$ which is a subset of the set of states on $\mathcal{A}$ and has the property of being closed under finite convex combinations and operations induced by elements of $\mathcal{U}_{\mathrm{alg}}^{\infty}$, i.e. if $\varphi \in \mathcal{S}$ and $A \in \mathcal{U}_{\mathrm{alg}}^{\infty}$, then the state $\varphi^{A}$ on $\mathcal{A}$ defined by

$$
\varphi^{A}(B):=\varphi\left(A^{*} B A\right) / \varphi\left(A^{*} A\right), \quad B \in \mathcal{A},
$$

is also contained in $\mathcal{S}$. We denote by $\mathcal{V}$ the vector-space generated by $\mathcal{S}$ and $\mathcal{U}_{\mathrm{alg}}^{\infty}$, that is, the subset of all elements $\ell$ in the continuous dual space $\mathcal{A}^{*}$ of $\mathcal{A}$ that arise as finite linear combinations of elements of $\mathcal{S}$ operated upon by elements of $\mathcal{U}_{\mathrm{alg}}^{\infty}$ from right and left:

$$
\ell(B)=\sum_{i=1}^{n} \varphi_{i}\left(A_{i} B C_{i}\right) \quad n \in \mathbb{N}, A_{i}, B_{i} \in \mathcal{U}_{\mathrm{alg}}^{\infty} \text { and } \varphi_{i} \in \mathcal{S}
$$

The energy density of the system is then defined to be a linear map $\varrho$ taking elements in $\mathcal{V}$ to $C^{1}$-functions on $\mathbb{R} \times \Sigma$. That is, given $\ell \in \mathcal{V}$, then $\varrho[\ell]$ is a $C^{1}$-function on $\mathbb{R} \times \Sigma$, 
and the assignment $\ell \mapsto \boldsymbol{\varrho}[\ell]$ is linear. We will find it convenient to write $\ell(\boldsymbol{\varrho}(t, \underline{x}))$ for $\varrho[\ell](t, \underline{x})$. Moreover, we will write $\ell([\boldsymbol{\varrho}(t, \underline{x}), A])$ to denote $\varrho\left[\ell_{A}-{ }_{A} \ell\right](t, \underline{x})$, where the functionals $\ell_{A}$ and ${ }_{A} \ell$ are given by

$$
\ell_{A}(B)=\ell(B A), \quad{ }_{A} \ell(B)=\ell(A B) .
$$

The quantity $\varphi(\varrho(t, \underline{x}))$ ought to be viewed as the expectation value of the energy-density with respect to the preferred time-coordinate in the state $\varphi \in \mathcal{S}$ at $(t, \underline{x})$. The following assumptions are in line with this point of view:

(i) It will be assumed that

$$
\int_{\Sigma} d \mu(\underline{x}) \ell\left(\left[\varrho(t, \underline{x}), \alpha_{t}(A)\right]\right)=-i \frac{d}{d t} \ell\left(\alpha_{t}(A)\right), \quad A \in \mathcal{U}_{\mathrm{alg}}^{\infty}, \ell \in \mathcal{V} .
$$

(ii) It will also be assumed that the integrated generating term of the energy density is conserved:

$$
\frac{d}{d t} \int_{\Sigma} d \mu(\underline{x}) \ell([\varrho(t, \underline{x}), A])=0, \quad A \in \mathcal{U}_{\mathrm{alg}}^{\infty}, \ell \in \mathcal{V}
$$

We note that, in the presence of (i), condition (ii) may be equivalently expressed by saying that the generators of the time-evolutions are independent of $t$,

$$
\frac{d}{d t} \ell\left(\alpha_{t}(A)\right)=\ell\left(\delta\left(\alpha_{t}(A)\right)\right), \quad A \in \mathcal{U}_{\mathrm{alg}}^{\infty}, \ell \in \mathcal{V}
$$

With the assumptions formulated so far, the notion of quantum weak energy inequality in the present general setting can now be precisely defined.

Definition 2.1 Assume that a physical system on the static spacetime $\mathbb{R} \times \Sigma$ modelled by $\mathcal{A},\left\{\alpha_{t}\right\}_{t \in \mathbb{R}}, \mathcal{W}^{\infty}, \mathcal{A}^{\infty}, \mathcal{S}$ and $\varrho$ with the properties stated above is given, and let $\omega \in \mathcal{S}$.

(a) We say that $\omega$ fulfills a static quantum weak energy inequality with respect to $\mathcal{S}$ if there is for each real-valued $g \in C_{0}^{\infty}(\mathbb{R})$ a locally integrable ${ }^{4}$ non-negative function $\Sigma \ni \underline{x} \mapsto q(g ; \underline{x})$ such that

$$
\int_{\mathbb{R}} d t g^{2}(t) \varphi(\underline{\varrho}(t, \underline{x}))-\int_{\mathbb{R}} d t g^{2}(t) \omega(\underline{\varrho}(t, \underline{x})) \geq-q(g ; \underline{x})
$$

holds for all states $\varphi \in \mathcal{S}$ and all $\underline{x} \in \Sigma$.

(b) We say that $\omega$ fulfills a limiting static QWEI (with respect to $\mathcal{S}$ ) if $\omega$ fulfills the static QWEI (2.2), and in addition each $\underline{x} \in \Sigma$ has an open neighbourhood $U$ such that

$$
\Gamma_{U}:=\sup _{g} \limsup _{\lambda \rightarrow 0^{+}} \frac{1}{\left\|g_{\lambda}^{2}\right\|_{L^{1}}} \int_{U} d \mu\left(\underline{x}^{\prime}\right) q\left(g_{\lambda} ; \underline{x}^{\prime}\right)<\infty
$$

where the supremum is taken over real-valued $g \in C_{0}^{\infty}(\mathbb{R})$ with $\left\|g^{2}\right\|_{L^{1}} \neq 0$, and $g_{\lambda}(t)=g(\lambda t)$.

\footnotetext{
${ }^{4}$ That is, each $\underline{x} \in \Sigma$ should have an open neighbourhood on which $q(g ; \cdot)$ is integrable.
} 
(c) A state $\omega \in \mathcal{S}$ will be called quiescent (with respect to $\mathcal{S}$ ) if $\omega$ fulfills a limiting static $Q W E I$ in which each $\Gamma_{U}=0$. (Since $q(g, \underline{x})$ is non-negative this is in fact equivalent to the assertion that each $\underline{x} \in \Sigma$ has an open neighbourhood $U$ such that

$$
\lim _{\lambda \rightarrow 0^{+}} \lambda \int_{U} d \mu\left(\underline{x}^{\prime}\right) q\left(g_{\lambda} ; \underline{x}^{\prime}\right)=0
$$

for each real-valued $g \in C_{0}^{\infty}(\mathbb{R})$.)

Remarks.

(i) It is easy to see that, if there exists a state $\omega \in \mathcal{S}$ fulfilling a static QWEI with respect to $\mathcal{S}$, then all states $\omega^{\prime} \in \mathcal{S}$ satisfy a static QWEI with respect to $\mathcal{S}$ as well. Therefore, it is actually more appropriate to say that the set of states $\mathcal{S}$ fulfills a static QWEI. Within the class of states $\mathcal{S}$, the condition of static QWEI is thus independent of the individually chosen state. In contrast, conditions (b) and (c) are state-dependent.

(ii) The element $\underline{x} \in \Sigma$ appearing in $q(g ; \underline{x})$ ought to viewed as a label for the timelike curve $\gamma_{\underline{x}}(t)=\tau_{t}((0, \underline{x}))$, i.e. the orbit of the point $(0, \underline{x}) \in M$ (identified with $\underline{x} \in \Sigma$ ) under the one-parametric group of time-shifts. Thus $q(g ; \underline{x})$ (or rather, $\tilde{q}(g ; \underline{x})=q(g ; \underline{x})+$ $\left.\int d t g^{2}(t) \omega(\varrho(t, \underline{x}))\right)$ in (2.2) plays exactly the role of $c_{g, \gamma_{\underline{x}}}$ in (1.1).

(iii) The condition (a) given above is a purely local statement about the dynamical system, and (b) and (c) are limits thereof which are still "spatially local". However, since $\Sigma$ is compact and $q(g ; \cdot)$ is non-negative, one may easily draw global consequences: if a static QWEI holds, then $q(g ; \cdot) \in L^{1}(\Sigma, d \mu)$, while if a limiting static QWEI holds then (2.3) also holds for $U=\Sigma$, with $\Gamma_{\Sigma}=0$ in the quiescent case. (Conversely, these global properties of course imply the local statements given above.) It is these global statements which will appear in the arguments presented below.

(iv) It will be shown in Section 4 that any state for the scalar Klein-Gordon field on a static, globally hyperbolic spacetime possessing a two-point function that is stationary and of Hadamard form fulfills a limiting static QWEI with

$$
q(g ; \underline{x})=\int_{\mathbb{R}} d u|\widehat{g}(u)|^{2} Q(u, \underline{x})
$$

where $\widehat{g}$ denotes the Fourier-transform of $g$, and $Q$ is a non-negative measurable function on $\mathbb{R} \times \Sigma$ so that $Q(u, \underline{x})$ is polynomially bounded in $u$ for each fixed $\underline{x} \in \Sigma$. Moreover, $Q(u, \underline{x})$ is $d \mu$-integrable over $\Sigma$ with respect to $\underline{x}$ for each $u \in \mathbb{R}$, and $\int_{\Sigma} d \mu(\underline{x}) Q(u, \underline{x})$ is polynomially bounded in $u$. This then implies that all states of the scalar KleinGordon field on a stationary, globally hyperbolic spacetime whose two-point functions are of Hadamard form (or, synonymously, satisfy the microlocal spectrum condition) fulfill a static QWEI.

We have now collected all the assumptions relevant for the present section. Before presenting our results on passivity properties of states satisfying static weak energy inequalities for systems obeying the assumptions given above, we put on record an auxiliary lemma. Recall (cf. Introduction) that $\mathcal{U}_{0}(\delta)$ denotes the set of all unitaries in $D(\delta)$ which are continuously connected to the unit $\mathbf{1}$, i.e. those unitaries $U$ in $D(\delta)$ so that there is a norm-continuous curve $[0,1] \ni t \mapsto U(t)$ of unitaries in $\mathcal{A}$ so that $U(0)=\mathbf{1}, U(1)=U$. 
Lemma $2.2 \mathcal{U}^{\infty}$ is dense in $\mathcal{U}_{0}(\delta)$ with respect to the graph-norm of $\delta$; i.e. for each $U \in \mathcal{U}_{0}(\delta)$ there is a sequence $U_{n} \in \mathcal{U}^{\infty}, n \in \mathbb{N}$, with

$$
\left\|U_{n}-U\right\|+\left\|\delta\left(U_{n}\right)-\delta(U)\right\| \rightarrow 0 \text { as } n \rightarrow \infty
$$

Proof. Let $A$ be a hermitean element in $D(\delta)$. We show that there is a sequence of hermitean elements in $\mathcal{A}^{\infty}$ approximating $A$ in the graph-norm of $\delta$. To this end, pick $\epsilon>0$ arbitrarily. Then choose some real-valued, non-zero $f \in C_{0}^{\infty}(\mathbb{R})$ so that

$$
\left\|\alpha_{f} A-A\right\|+\left\|\alpha_{f} \delta(A)-\delta(A)\right\|<\epsilon / 2
$$

Because $\mathcal{W}^{\infty} \subset \mathcal{A}^{\infty}$ is norm-dense in $D(\delta)$ and stable under taking adjoints, one may also choose some hermitean $A_{\epsilon} \in \mathcal{W}^{\infty}$ so that

$$
\left\|A_{\epsilon}-A\right\|<\frac{\epsilon}{2}\left(\|f\|_{L^{1}}+\|\dot{f}\|_{L^{1}}\right)^{-1}
$$

where $\dot{f}$ is the derivative of $f$. Then one estimates

$$
\begin{aligned}
& \left\|\alpha_{f} A_{\epsilon}-A\right\|+\left\|\delta\left(a_{f} A_{\epsilon}\right)-\delta(A)\right\| \\
& \quad \leq\left\|\alpha_{f} A_{\epsilon}-\alpha_{f} A\right\|+\left\|\alpha_{f} A-A\right\|+\left\|\delta\left(\alpha_{f} A_{\epsilon}\right)-\delta\left(\alpha_{f} A\right)\right\|+\left\|\alpha_{f} \delta(A)-\delta(A)\right\| \\
& \quad \leq \epsilon / 2+\|f\|_{L^{1}}\left\|A_{\epsilon}-A\right\|+\|\dot{f}\|_{L^{1}}\left\|A_{\epsilon}-A\right\| \leq \epsilon
\end{aligned}
$$

by making use of $\alpha_{f} \delta(B)=\delta\left(\alpha_{f} B\right)=-\alpha_{\dot{f}}(B)$ for all $B \in D(\delta)$ and $\left\|\alpha_{g} B\right\| \leq\|g\|_{L^{1}}\|B\|$ for $g \in C_{0}^{\infty}(\mathbb{R})$. As $\mathcal{W}^{\infty}$ is by assumption stable under convolution with test-functions with respect to the dynamical automorphism group, this shows that each hermitean $A \in D(\delta)$ can be approximated by a sequence of hermitean elements in $\mathcal{W}^{\infty} \subset \mathcal{A}^{\infty}$ in the graph-norm of $\delta$.

As argued in the bottom part of page 279 in 33 (cf. also Thm. 5.4.28 in 7]), for each $U \in \mathcal{U}_{0}(\delta)$ there exist finitely many hermitean elements $A_{1}, \ldots, A_{N} \in D(\delta)$ with $\left\|A_{j}\right\|<\pi(j=1, \ldots, N)$ so that

$$
U=\mathrm{e}^{i A_{1}} \cdots \mathrm{e}^{i A_{N}}
$$

Since each $A_{j}$ may be approximated by a sequence of hermitean elements $A_{j}^{(n)}, n \in \mathbb{N}$, in $\mathcal{A}^{\infty}$, it is quite easy to see that also $U_{n}=\mathrm{e}^{i A_{1}^{(n)}} \cdots \mathrm{e}^{A_{N}^{(n)}}$ approximates $U$ in the graph-norm of $\delta$, and each $U_{n}$ is contained in $\mathcal{U}^{\infty}$. This proves the lemma.

Now, with the notation and assumptions introduced prior to Lemma 2.2, our first result reads as follows.

Theorem 2.3 Suppose that a state $\omega \in \mathcal{S}$ satisfies a static $Q W E I$. Then there exists a state $\omega^{p}$ on $\mathcal{A}$ which is passive for the automorphism group $\left\{\alpha_{t}\right\}_{t \in \mathbb{R}}$, i.e. it fulfills

$$
\frac{1}{i} \omega^{p}\left(U^{*} \delta(U)\right) \geq 0
$$

for all the unitaries $U$ in $\mathcal{U}_{0}(\delta)$.

Moreover, if $\omega$ is a quiescent state, then it is a passive state for the automorphism group $\left\{\alpha_{t}\right\}_{t \in \mathbb{R}}$. 
The proof of the first part of that result is based on a simple

Lemma 2.4 Let $\omega$ be a state on $\mathcal{A}$ so that

$$
c_{\omega}:=\inf _{U \in \mathcal{U}^{\infty}} \frac{1}{i} \omega\left(U^{*} \delta(U)\right)>-\infty,
$$

then there exists a state $\omega^{p}$ on $\mathcal{A}$ which is passive for $\left\{\alpha_{t}\right\}_{t \in \mathbb{R}}$.

Proof of Lemma 2.4 If $c_{\omega} \geq 0$, then $\omega$ is already passive. Therefore, we assume that $c_{\omega} \in(-\infty, 0)$. There exists a sequence $\widetilde{U}_{n} \in \mathcal{U}^{\infty}, n \in \mathbb{N}$, so that $\frac{1}{i} \lim _{n \rightarrow \infty} \omega\left(\widetilde{U}_{n}^{*} \delta\left(\widetilde{U}_{n}\right)\right)=$ $c_{\omega}$. The sequence of states $\omega_{n}, n \in \mathbb{N}$, on $\mathcal{A}$ given by $\omega_{n}(A)=\omega\left(\widetilde{U}_{n}^{*} A \widetilde{U}_{n}\right)$ possesses, by the Banach-Alaoglu-theorem [35], weak-* limit points, i.e. there is a state $\omega^{p}$ on $\mathcal{A}$ and a subnet $\left\{\omega_{n(\sigma)}\right\}_{\sigma \in S}$ of $\left\{\omega_{n}\right\}_{n \in \mathbb{N}}$ so that $\lim _{\sigma} \omega_{n(\sigma)}(A)=\omega^{p}(A)$ for all $A \in \mathcal{A}$. Abbreviating $\widetilde{U}_{n(\sigma)}$ by $\widetilde{U}_{\sigma}$, we obtain for all $U \in \mathcal{U}^{\infty}$

$$
\begin{aligned}
& \frac{1}{i} \omega^{p}\left(U^{*} \delta(U)\right)=\frac{1}{i} \lim _{\sigma} \omega\left(\widetilde{U}_{\sigma}^{*} U^{*} \delta(U) \widetilde{U}_{\sigma}\right) \\
& \quad=\frac{1}{i} \lim _{\sigma}\left(\omega\left(\left(U \widetilde{U}_{\sigma}\right)^{*} \delta\left(U \widetilde{U}_{\sigma}\right)\right)-\omega\left(\widetilde{U}_{\sigma}^{*} \delta\left(\widetilde{U}_{\sigma}\right)\right)\right) \\
& \quad \geq \liminf _{\sigma} \frac{1}{i} \omega\left(\left(U \widetilde{U}_{\sigma}\right)^{*} \delta\left(U \widetilde{U}_{\sigma}\right)\right)-\frac{1}{i} \lim _{\sigma} \omega\left(\widetilde{U}_{\sigma}^{*} \delta\left(\widetilde{U}_{\sigma}\right)\right) \\
& \quad \geq c_{\omega}-c_{\omega}=0 .
\end{aligned}
$$

In view of Lemma 2.2, this relation entails that

$$
\inf _{U \in \mathcal{U}_{0}(\delta)} \frac{1}{i} \omega^{p}\left(U^{*} \delta(U)\right) \geq 0
$$

showing that $\omega^{p}$ is passive.

Proof of Thm. 2.3 In view of conditions (i) and (ii) one obtains for all $U \in \mathcal{U}^{\infty}$ and any real $g \in C_{0}^{\infty}(\mathbb{R})$ with $\left\|g^{2}\right\|_{L^{1}}>0$

$$
\begin{aligned}
& \frac{1}{i} \omega\left(U^{*} \delta(U)\right)=\int_{\Sigma} d \mu(\underline{x}) \omega\left(U^{*}[\varrho(t, \underline{x}), U]\right) \\
& \quad=\frac{1}{\left\|g^{2}\right\|_{L^{1}}} \int_{\mathbb{R}} d t g^{2}(t) \int_{\Sigma} d \mu(\underline{x})\left(\omega\left(U^{*} \boldsymbol{\varrho}(t, \underline{x}) U\right)-\omega(\boldsymbol{\varrho}(t, \underline{x}))\right) \\
& \quad=\frac{1}{\left\|g^{2}\right\|_{L^{1}}} \int_{\Sigma} d \mu(\underline{x}) \int_{\mathbb{R}} d t g^{2}(t)\left(\omega\left(U^{*} \varrho(t, \underline{x}) U\right)-\omega(\boldsymbol{\varrho}(t, \underline{x}))\right) \\
& \quad \geq \frac{-1}{\left\|g^{2}\right\|_{L^{1}}} \int_{\Sigma} d \mu(\underline{x}) q(g ; \underline{x}) .
\end{aligned}
$$

If $\omega$ is quiescent, we replace $g$ by $g_{\lambda}$ in the last term and take the limit as $\lambda \rightarrow 0^{+}$ in order to conclude that $\omega$ is passive by Lemma 2.2. Otherwise, we nonetheless have $\inf _{U \in \mathcal{U}^{\infty}} i^{-1} \omega\left(U^{*} \delta(U)\right)>-\infty$; the existence of the passive state $\omega^{p}$ follows from Lemma 2.4 .

It is shown in [33] that if $\omega$ is a passive state which is weakly clustering in time (and noncentral) then $\omega$ is a ground state or a KMS-state at positive inverse temperature for the 
time-evolution $\left\{\alpha_{t}\right\}_{t \in \mathbb{R}}$. Here we add another observation, assuming that a state fulfilling a limiting static QWEI is weakly clustering in time for the integrated energy density. We denote by

$$
\ell\left(\varrho_{\Sigma}(t)\right)=\int_{\Sigma} d \mu(\underline{x}) \ell(\varrho(t, \underline{x})), \quad \ell \in \mathcal{V},
$$

the integrated energy density at time $t$ in the functional $\ell$, and we will say that a state $\omega \in \mathcal{S}$ is weakly clustering in time for the integrated energy density if for every $B \in \mathcal{U}_{\text {alg }}^{\infty}$ there exists a sequence $\left\{\lambda_{n}\right\}$ of positive real numbers converging to 0 and some real-valued $g \in C_{0}^{\infty}(\mathbb{R})$ with $\int d t g_{n}^{2}(t)=1$ so that

$$
\int_{\mathbb{R}} d t \lambda_{n} g^{2}\left(\lambda_{n} t\right)\left\{\omega\left(B \boldsymbol{\varrho}_{\Sigma}(t)\right)-\omega(B) \omega\left(\boldsymbol{\varrho}_{\Sigma}(t)\right)\right\} \rightarrow 0 \text { as } n \rightarrow \infty .
$$

We need another piece of notation. Recall that any state $\omega$ on a $C^{*}$-algebra $\mathcal{A}$ induces the so-called GNS-representation $(\mathcal{H}, \pi, \Omega)$ consisting of a Hilbert-space $\mathcal{H}$, a linear *representation $\pi$ of $\mathcal{A}$ by bounded linear operators on $\mathcal{H}$, and a unit vector $\Omega$ which is cyclic for $\pi(\mathcal{A})$ in the sense that $\pi(\mathcal{A}) \Omega$ is dense in $\mathcal{H}$, and which fulfills $\omega(A)=\langle\Omega, \pi(A) \Omega\rangle$ for all $A \in \mathcal{A}$. If in addition $\omega$ is invariant with respect to the automorphism group $\left\{\alpha_{t}\right\}_{t \in \mathbb{R}}$, $\omega \circ \alpha_{t}=\omega$, then there is a strongly continuous unitary group $V_{t}, t \in \mathbb{R}$, on $\mathcal{H}$ which implements the action of $\alpha_{t}, t \in \mathbb{R}$, in the GNS-representation: $V_{t} \pi(A) V_{t}^{*}=\pi\left(\alpha_{t}(A)\right)$, $A \in \mathcal{A}$; moreover, $V_{t} \Omega=\Omega$ for all $t$ (see, e.g. [6] for a thorough discussion of these matters). The selfadjoint operator $H$ in $\mathcal{H}$ with $V_{t}=\mathrm{e}^{i H t}$ is called the generator of the implementing unitary group.

Theorem 2.5 Let $\omega \in \mathcal{S}$ be a state which is weakly clustering in time for the integrated energy density.

(a) If $\omega$ is a quiescent state, then it follows that $\omega$ is a ground state for $\left\{\alpha_{t}\right\}_{t \in \mathbb{R}}$.

(b) If $\omega$ is invariant with respect to the automorphism group $\left\{\alpha_{t}\right\}_{t \in \mathbb{R}}$ and fulfills a limiting static $Q W E I$, then the generator $H$ of the implementing unitary group is bounded below by $-\Gamma_{\Sigma}$, defined by Eq. (2.3) [see also remark (iii) following Def. 2.1].

Proof. Choose any $A \in \mathcal{U}_{\mathrm{alg}}^{\infty}$ with $\omega\left(A^{*} A\right)=1$ and let $\left\{\lambda_{n}\right\}$ be a sequence of positive real numbers converging to 0 as well as $g \in C_{0}^{\infty}(\mathbb{R})$ with $\left\|g^{2}\right\|_{L^{1}}=1$ such that (2.4) holds with $B=A^{*} A$. Then, setting $g_{n}(t)=\sqrt{\lambda_{n}} g\left(\lambda_{n} t\right)$ it follows that

$$
\epsilon_{n}:=\int_{\mathbb{R}} d t g_{n}^{2}(t)\left\{\omega\left(\varrho_{\Sigma}(t)\right)-\omega\left(A^{*} A \varrho_{\Sigma}(t)\right)\right\} \rightarrow 0 \text { as } n \rightarrow \infty .
$$

Recalling that $\omega\left(A^{*}\left[\varrho_{\Sigma}(t), A\right]\right)$ is independent of $t$, there results the following chain of relations:

$$
\begin{aligned}
\frac{1}{i} \omega\left(A^{*} \delta(A)\right) & =\omega\left(A^{*} \boldsymbol{\varrho}_{\Sigma}(t) A\right)-\omega\left(A^{*} A \boldsymbol{\varrho}_{\Sigma}(t)\right) \\
& =\int_{\mathbb{R}} d t g_{n}^{2}(t)\left\{\omega\left(A^{*} \varrho_{\Sigma}(t) A\right)-\omega\left(A^{*} A \boldsymbol{\varrho}_{\Sigma}(t)\right)\right\} \\
& =\int_{\mathbb{R}} d t g_{n}^{2}(t)\left\{\omega\left(A^{*} \varrho_{\Sigma}(t) A\right)-\omega\left(\boldsymbol{\varrho}_{\Sigma}(t)\right)\right\}+\epsilon_{n} \\
& \geq-\int_{\Sigma} d \mu(\underline{x}) \frac{q\left(g_{\lambda_{n}} ; \underline{x}\right)}{\left\|g_{\lambda_{n}}^{2}\right\|_{L^{1}}}+\epsilon_{n} .
\end{aligned}
$$


In the limit $n \rightarrow \infty$, this gives

$$
\frac{1}{i} \omega\left(A^{*} \delta(A)\right) \geq-\limsup _{\lambda \rightarrow 0^{+}} \int_{\Sigma} d \mu(\underline{x}) \frac{q\left(g_{\lambda} ; \underline{x}\right)}{\left\|g_{\lambda}^{2}\right\|_{L^{1}}} \geq-\Gamma_{\Sigma},
$$

since in either case $\omega$ obeys a limiting static QWEI.

Case $(a)$. As $\omega$ is assumed to be quiescent, $\Gamma_{\Sigma}=0$, so

$$
\frac{1}{i} \omega\left(A^{*} \delta(A)\right) \geq 0
$$

holds for all $A \in \mathcal{U}_{\mathrm{alg}}^{\infty}$ with $\omega\left(A^{*} A\right)=1$, hence also for all $A \in \mathcal{U}_{\mathrm{alg}}^{\infty}$. The proof of Lemma 2.2 shows that the closure of $\mathcal{U}_{\text {alg }}^{\infty}$ in the graph-norm of $\delta$ contains $D(\delta)$, and thus $\omega$ is a ground state.

Case $(b)$. In view of the definition of $\Gamma_{\Sigma}$, Eq. (2.5) entails

$$
\langle A \Omega, H A \Omega\rangle=\frac{1}{i} \omega\left(A^{*} \delta(A)\right) \geq-\Gamma_{\Sigma}
$$

for all $A \in \mathcal{U}_{\text {alg }}^{\infty}$ with $\|A \Omega\|^{2}=1$, and hence also for all $A \in D(\delta)$ with $\|A \Omega\|^{2}=1$. Since $D(\delta) \Omega$ contains a core for $H$, it follows that $H$ is bounded below by $-\Gamma_{\Sigma}$.

Thm. 2.3 shows that the existence of passive states for the given system may be deduced from the existence of states satisfying a static quantum weak energy inequality; however, this result leaves room for further sharpening. In particular, one would like to know if the passive state $\omega^{p}$ is normal to the state $\omega$ assumed to fulfill a static quantum weak energy inequality. It turns out that such an assertion can be made under the assumption that $\omega$ satisfies a certain condition of "energy compactness". Let us fix the required assumptions in detail.

We assume that we are given a Hilbert-space $\mathcal{H}$ and a strongly continuous unitary group $\left\{V_{t}\right\}_{t \in \mathbb{R}}$ on $\mathcal{H}$ with selfadjoint generator $H$, i.e. $V_{t}=\mathrm{e}^{i t H}$. Furthermore, we assume that there is a $C^{*}$-subalgebra $\mathcal{A}$ of $\mathcal{L}(\mathcal{H})$ which contains the unit operator $\mathbf{1}$ and is left invariant under the automorphism group $\alpha_{t}:=\operatorname{Ad} V_{t}, t \in \mathbb{R}$. In addition, it will be assumed that $\left\{\alpha_{t}\right\}_{t \in \mathbb{R}}$ acts strongly continuously on the elements of $\mathcal{A}$, and that, for each $E \geq 0$, the spectral projector $P_{E}$ of $H$ corresponding to the spectral interval $[-E, E]$, is also contained in $\mathcal{A}$. With these conventions, the definitions of $\delta, D(\delta), \mathcal{U}^{\infty}$ and $\mathcal{U}_{\mathrm{alg}}^{\infty}$, etc., are as before. A vector $\Omega \in \mathcal{H}$ will be said to be energy-compact if for each finite $E>0$ the set $P_{E} \mathcal{A}_{(1)} \Omega$ is a pre-compact subset of $\mathcal{H}$. Here $\mathcal{A}_{(1)}$ is the set of all elements in $\mathcal{A}$ whose norm is bounded by 1 , and we recall that a subset $\mathcal{J}$ of $\mathcal{H}$ is called pre-compact if each sequence $\left\{\chi_{n}\right\}_{n \in \mathbb{N}}$ in $\mathcal{J}$ possesses a sub-sequence $\left\{\chi_{n(k)}\right\}_{k \in \mathbb{N}}$ which converges strongly to some element $\chi \in \mathcal{H}$, i.e. $\left\|\chi_{n(k)}-\chi\right\| \rightarrow 0$ as $k \rightarrow \infty$.

Energy-compactness conditions were introduced by Haag and Swieca in quantum field theory [24; they impose restrictions on the energy-level density of quantum states. The original approach of Haag and Swieca has been considerably extended and refined to socalled "nuclearity conditions" in quantum field theory in a series of works by Buchholz and collaborators. It is interesting to note that there is a close connection between such nuclearity conditions and decent thermodynamical properties of quantum field systems. 
In fact, this was one of the central motivations for the introduction of nuclearity conditions in [4. We recommend [23] and 4, 5, 41, 42] for further discussions and references on that subject, and we would like to refer also to [1, 22, 28, 29] for some recent developments. Here, we just mention that related energy-compactness conditions have been shown to hold for energy-ground states (vacuum states) in several quantum field theoretical models (see the quoted works, and references quoted therein); notably, they have also been established for linear quantum field theories on ultrastatic curved spacetimes [8, 44].

The assumptions listed above now lead to

Theorem 2.6 Let $\Omega$ be a unit vector in $\mathcal{H}$ which is energy-compact, and let $\omega(A):=$ $\langle\Omega, A \Omega\rangle$ be the vector-state on $\mathcal{A}$ induced by $\Omega$. If

$$
c_{\omega}:=\inf _{U \in \mathcal{U}_{0}(\delta)} \frac{1}{i} \omega\left(U^{*} \delta(U)\right)>-\infty
$$

then there exists a unit vector $\Omega^{p} \in \overline{\mathcal{A} \Omega}$ so that the vector-state $\omega^{p}$ induced by $\Omega^{p}$ is passive.

Proof. There is a sequence $\widetilde{U}_{n}, n \in \mathbb{N}$, in $\mathcal{U}^{\infty}$ so that $\lim _{n \rightarrow \infty} \frac{1}{i} \omega\left(\widetilde{U}_{n}^{*} \delta\left(\widetilde{U}_{n}\right)\right)=c_{\omega}$. Since $\widetilde{U}_{n} \Omega, n \in \mathbb{N}$, is a bounded sequence in $\mathcal{H}$ and possesses a weakly converging sub-sequence, it is no loss of generality to assume that $w-\lim _{n \rightarrow \infty} \widetilde{U}_{n} \Omega=\Omega^{p}$ for some unit vector $\Omega^{p} \in \mathcal{H}$. Now let $A_{1}, \ldots, A_{N}$ be finitely many hermitean elements in $D^{\infty}(\delta)$, define $U$ as in the proof of Lemma 2.2, and write

$$
U_{E}:=\mathrm{e}^{i P_{E} A_{1} P_{E}} \ldots \mathrm{e}^{i P_{E} A_{N} P_{E}}, \quad E>0 .
$$

Then it holds that $U_{E}=\mathbf{1}+G_{E}$ where $\left\|G_{E}\right\| \leq 2$ and

$$
G_{E}=P_{E} G_{E} P_{E}
$$

Introducing the bounded operator $F_{E}:=\left(\mathbf{1}+G_{E}^{*}\right)\left[H, G_{E}\right]=-i U_{E}^{*} \delta\left(U_{E}\right)$, we note that $F_{E}=P_{E} F_{E} P_{E}$. Moreover, by assumption $P_{E} \mathcal{A}_{(1)} \Omega$ is a pre-compact set, and therefore the sequence of vectors $P_{E} \widetilde{U}_{n} \Omega \in P_{E} \mathcal{A}_{(1)} \Omega$ possesses a subsequence $P_{E} \widetilde{U}_{n(k)} \Omega$ converging strongly to $P_{E} \Omega^{p}$ (since $\widetilde{U}_{n} \Omega$ is already known to converge weakly to $\Omega^{p}$ ). This allows us to write $\left(\right.$ with $\left.\omega^{p}(\cdot)=\left\langle\Omega^{p}, \cdot \Omega^{p}\right\rangle\right)$

$$
\begin{aligned}
& \frac{1}{i} \omega^{p}\left(U_{E}^{*} \delta\left(U_{E}\right)\right)=\omega^{p}\left(F_{E}\right)=\omega^{p}\left(P_{E} F_{E} P_{E}\right) \\
& \quad=\lim _{k \rightarrow \infty}\left\langle P_{E} \widetilde{U}_{n(k)} \Omega, F_{E} P_{E} \widetilde{U}_{n(k)} \Omega\right\rangle \\
& \quad=\lim _{k \rightarrow \infty}\left\langle\widetilde{U}_{n(k)} \Omega, F_{E} \widetilde{U}_{n(k)} \Omega\right\rangle=\lim _{k \rightarrow \infty} \frac{1}{i} \omega\left(\widetilde{U}_{n(k)}^{*} U_{E}^{*} \delta\left(U_{E}\right) \widetilde{U}_{n(k)}\right) \\
& \quad=\frac{1}{i} \lim _{k \rightarrow \infty}\left\{\omega\left(\left(U_{E} \widetilde{U}_{n(k)}\right)^{*} \delta\left(U_{E} \widetilde{U}_{n(k)}\right)\right)-\omega\left(\widetilde{U}_{n(k)}^{*} \delta\left(\widetilde{U}_{n(k)}\right)\right)\right\} \\
& \quad \geq \liminf _{k \rightarrow \infty} \frac{1}{i} \omega\left(\left(U_{E} \widetilde{U}_{n(k)}\right)^{*} \delta\left(U_{E} \widetilde{U}_{n(k)}\right)\right)-\lim _{k \rightarrow \infty} \frac{1}{i} \omega\left(\widetilde{U}_{n(k)}^{*} \delta\left(\widetilde{U}_{n(k)}\right)\right) \\
& \quad \geq c_{\omega}-c_{\omega}=0 .
\end{aligned}
$$


This shows that $\frac{1}{i} \omega^{p}\left(U_{E}^{*} \delta\left(U_{E}\right)\right) \geq 0$ for all $E>0$, and it is easy to check that also $\lim _{E \rightarrow \infty} \omega^{p}\left(U_{E}^{*} \delta\left(U_{E}\right)\right)=\omega^{p}\left(U^{*} \delta(U)\right)$. Therefore we conclude that

$$
\inf _{U \in \mathcal{U}_{0}(\delta)} \frac{1}{i} \omega^{p}\left(U^{*} \delta(U)\right) \geq 0
$$

proving passivity of $\omega^{p}$.

Under the assumptions of Thm. 2.6 one finds, by combining it with Thm. 2.3, the following

Corollary 2.7 Let $\Omega$ be a unit vector in $\mathcal{H}$ which is energy compact, and suppose that the corresponding vector state $\omega$ fulfills a static quantum weak energy inequality. Then there exists a unit vector in $\overline{\mathcal{A} \Omega}$ inducing a passive state for $\left\{\alpha_{t}\right\}_{t \in \mathbb{R}}$.

\section{Some techniques from microlocal analysis}

In the next section, we will show that the real scalar field on a static spacetime is a system satisfying the assumptions made in the previous section and to which our results therefore apply. Our principal tools will be drawn from microlocal analysis [25, 26, which provides powerful and geometrically natural techniques for dealing with the singular structure of distributions. We now proceed to describe the microlocal analysis used in Sec. 4 and the Appendix, adopting a slightly more intrinsic approach than usual, which avoids the explicit introduction of coordinates.

Let $X$ be a smooth manifold and denote by $\dot{T}^{*} X=T^{*} X \backslash z$ the cotangent bundle of $X$ with its zero section $Z$ removed. Given a distribution $u \in \mathscr{D}^{\prime}(X)$, an element $(x, k) \in \dot{T}^{*} X$ is called a regular directed point for $u$ if there exists a set $\mathcal{O}$ and a map $\phi$ obeying $^{5}$

(A) $\mathcal{O}$ is an open neighbourhood of $x$, and $\phi: \mathcal{O} \rightarrow T_{x} X$ is a smooth map with nondegenerate tangent mapping $T \phi$ obeying $T \phi(x)=\mathrm{id}_{T_{x} X}$

and such that

(B) there exists $\chi \in C_{0}^{\infty}(\mathcal{O})$ with $\chi(x) \neq 0$ and a neighbourhood $E$ of $k$ in $T_{x}^{*} X$ such that

$$
\lambda^{N} \sup _{\ell \in E}\left|u\left(\chi e^{i \lambda\langle\ell, \phi\rangle}\right)\right| \rightarrow 0 \quad \text { as } \lambda \rightarrow+\infty \text { for each } N \in \mathbb{N}_{0} .
$$

The quantity inside the modulus signs can be interpreted as a local Fourier transform ${ }^{6}$ of $\chi u$ evaluated at $\lambda \ell$ : To see this, it may help to write this quantity as

$$
u\left(\chi e^{i \lambda\langle\ell, \phi\rangle}\right)=\int d \operatorname{vol}(x) \chi(x) u(x) e^{i \lambda \ell_{a} \phi^{a}(x)},
$$

\footnotetext{
${ }^{5}$ Condition (A) permits the introduction of coordinates on $\mathcal{O}$ by $y^{\mu}=\left\langle\zeta^{\mu}, \phi(y)\right\rangle$ where $\zeta^{\mu}$ is any fixed basis for $T_{x}^{*} X$, but such coordinates will rarely be necessary in our discussion.

${ }^{6}$ Throughout this paper, we adopt the nonstandard convention $\widehat{f}(k)=\int d^{n} x f(x) e^{i k \cdot x}$ for the Fourier transform on $\mathbb{R}^{n}$.
} 
where $u(x)$ is the distributional kernel of $u$ with respect to some volume measure $d$ vol on $X$.

As is well known, the Fourier transform of any smooth compactly supported function decays rapidly at infinity, so every $(x, k) \in \dot{T}^{*} X$ is regular directed for any distribution which may be identified with a smooth function. It may also be shown that if $(x, k)$ is a regular directed point for $u$ then condition (B) will be satisfied for any pair $(\mathcal{O}, \phi)$ obeying condition (A). Moreover, if condition (B) holds, it continues to hold if $\chi$ is replaced by $\psi \chi$ for any $\psi \in C_{0}^{\infty}(X)$. We are now in a position to define the central object of the theory.

Definition 3.1 The wave-front set $\mathrm{WF}(u)$ of a distribution $u \in \mathscr{D}(X)$ is the complement in $\dot{T}^{*} X$ of the set of regular directed points for $u$.

Two important facts will be used extensively in this work: first, that the wave-front set of a distribution $u$ is empty if and only if $u$ can be identified with a smooth function; second, if $P$ is a partial differential operator with smooth coefficients then $\mathrm{WF}(P u) \subset \mathrm{WF}(u)$.

Microlocal techniques have recently found many applications in the theory of quantum fields on curved spacetimes following Radzikowski's discovery [34 that the Hadamard condition (see Sec. 4.2) can be reformulated as a condition on the wave-front set of the two-point function of the field. Recently, this criterion has been simplified by Strohmaier, Wollenberg and Verch [40, who consider a generalisation of the wave-front set to distributions taking values in a Hilbert space. Generalising this slightly further, if $(B,\|\cdot\|)$ is a Banach space, let $\mathscr{D}^{\prime}(X, B)$ be the space of distributions on $X$ taking values in $B$, i.e., linear functionals $T: \mathscr{D}(X) \rightarrow B$ such that $f \rightarrow 0$ in $\mathscr{D}(X)$ implies $\|T(f)\| \rightarrow 0$. Then the wave-front set of $T$ may be defined as for scalar valued distributions, but with the Banach space norm replacing the modulus signs in (3.1). With this definition, a convenient calculus may be constructed as follows.

Proposition 3.2 Let $X$ be a $C^{\infty}$-manifold, $(\mathcal{H},\langle\cdot, \cdot\rangle)$ a Hilbert space and $\left(B_{i},\|\cdot\|_{i}\right)$ be Banach spaces $(i=1,2)$.

(i) If $T \in \mathscr{D}^{\prime}\left(X, B_{1}\right)$ and $S: \mathscr{D}(X) \rightarrow B_{2}$ is a linear map obeying

$$
\|S(f)\|_{2} \leq c\|T(f)\|_{1}, \quad f \in \mathscr{D}(X)
$$

for some $c \geq 0$, then $S \in \mathscr{D}^{\prime}\left(X, B_{2}\right)$ and $\mathrm{WF}(S) \subset \mathrm{WF}(T)$.

(ii) If $T \in \mathscr{D}^{\prime}(X, \mathcal{H})$ and $\psi \in \mathcal{H}$ then $f \mapsto\langle\psi, T(f)\rangle$ and $f \mapsto\langle T(\bar{f}), \psi\rangle$ define scalar distributions in $\mathscr{D}^{\prime}(X)$ with

$$
\mathrm{WF}(\langle\psi, T(\cdot)\rangle) \subset \mathrm{WF}(T(\cdot)) \text { and } \operatorname{WF}(\langle T(-), \psi\rangle) \subset \mathrm{WF}(T(\cdot))^{\dagger},
$$

where, for any $\Gamma \subset T^{*} X, \Gamma^{\dagger}=\left\{(x, k) \in T^{*} X \mid(x,-k) \in \Gamma\right\}$.

(iii) If $S, T \in \mathscr{D}^{\prime}(X, \mathcal{H})$ then $U:(f, g) \mapsto\langle S(\bar{f}), T(g)\rangle$ defines $U \in \mathscr{D}^{\prime}(X \times X)$ with

$$
\mathrm{WF}(U) \subset\left(\mathrm{WF}(S)^{\dagger} \cup \mathcal{Z}\right) \times(z \cup \mathrm{WF}(T)) .
$$

Proof: (i) The bound $\|S(f)\|_{2} \leq c\|T(f)\|_{1}$ implies that $S \in \mathscr{D}^{\prime}\left(X, B_{2}\right)$ and moreover that any regular directed point for $T$ is a regular directed point for $S$. The result follows on taking complements.

(ii) The statements regarding $f \mapsto\langle\psi, T(f)\rangle$ follow immediately from (i) and the CauchySchwarz inequality. To study $f \mapsto\langle T(\bar{f}), \psi\rangle$, it is convenient to prove an auxiliary result first. 
Lemma 3.3 Suppose $B$ is a Banach space equipped with a conjugation $\Gamma$ and that $S \in$ $\mathscr{D}^{\prime}(X, B)$. Defining

$$
S^{\dagger}(f)=\Gamma S(\bar{f}), \quad f \in \mathscr{D}(X),
$$

we have $S^{\dagger} \in \mathscr{D}^{\prime}(X, B)$ and $\mathrm{WF}\left(S^{\dagger}\right)=\mathrm{WF}(S)^{\dagger}$.

Proof: Since $\left\|S^{\dagger}(f)\right\|=\|S(\bar{f})\|$ and $f \rightarrow 0$ if and only if $\bar{f} \rightarrow 0$, we have $S^{\dagger} \in \mathscr{D}^{\prime}(X, B)$. Furthermore, it is easy to see that $(x, k)$ is a regular direction for $S,(x,-k)$ is a regular direction for $S^{\dagger}$, so $\mathrm{WF}\left(S^{\dagger}\right) \subset \mathrm{WF}(S)^{\dagger}$; since $\left(S^{\dagger}\right)^{\dagger}=S$, we must in fact have equality.

Now any Hilbert space admits a conjugation, and the Cauchy-Schwarz inequality gives

$$
|\langle T(\bar{f}), \psi\rangle| \leq\|\psi\|\|T(\bar{f})\|=\|\psi\|\left\|T^{\dagger}(f)\right\|,
$$

so we apply (i) and Lemma 3.3 to complete the proof of (ii).

(iii) Applying Cauchy-Schwarz,

$$
|U(f, g)| \leq\|S(\bar{f})\|\|T(g)\|=\left\|S(f)^{\dagger}\right\|\|T(g)\|=\left\|\left(S^{\dagger} \otimes T\right)(f, g)\right\|,
$$

where $S^{\dagger} \otimes T \in \mathscr{D}^{\prime}(X \times X, \mathcal{H} \otimes \mathcal{H})$ is defined by $\left(S^{\dagger} \otimes T\right)(f, g)=S^{\dagger}(f) \otimes T(g)$. The same arguments which bound the wave-front set of a tensor product of scalar distributions may be used to show that

$$
\mathrm{WF}\left(S^{\dagger} \otimes T\right) \subset\left(\mathrm{WF}\left(S^{\dagger}\right) \cup z\right) \times(z \cup \mathrm{WF}(T))
$$

[actually, there is a tighter bound than this]; using (i) and Lemma 3.3 the required result is obtained.

A further important property required below is the behaviour of distributions under the pull-back operation. Let $X_{1}$ and $X_{2}$ be smooth manifolds and let $\chi: X_{1} \rightarrow X_{2}$ be a $C^{\infty}$-map. To this map one can associate its conormal bundle $N_{\chi} \subset T^{*} X_{2}$ where, by definition, $(y, \eta)$ is in $N_{\chi}$ if and only if there is $x \in X_{1}$ with $y=\chi(x)$ and ${ }^{t} T \chi(x) \eta=0$, ${ }^{t} T \chi(x)$ denoting the transpose of the tangent map of $\chi$ at $x$.

If $F: X_{2} \rightarrow \mathbb{C}$ is a smooth map, one obtains via the pull-back by $\chi$ a smooth map $\chi^{*} F=F \circ \chi: X_{1} \rightarrow \mathbb{C}$. It can be shown (cf. Thm. 8.2.4 in [25]) that one can (uniquely) extend the pull-back operation to distributions $u \in \mathscr{D}^{\prime}\left(X_{2}\right)-$ through approximating distributions by test functions — provided that

$$
N_{\chi} \cap \mathrm{WF}(u)=\emptyset .
$$

In this case, the pull-back $\chi^{*} u$ of $u$ by $\chi$ has the property

$$
\mathrm{WF}\left(\chi^{*} u\right)=\chi^{*} \mathrm{WF}(u) \text {, }
$$

where for any subset $V \subset T^{*} X_{2}, \chi^{*} V$ is the subset of $T^{*} X_{1}$ defined as follows:

$$
\chi^{*} V=\left\{\left(x,{ }^{t} T \chi(x) \eta\right):(f(x), \eta) \in V\right\} .
$$

Moreover (see again Thm. 8.2.4 in [25]), $\chi^{*}$ induces a continuous linear map from $\mathscr{D}_{V}^{\prime}\left(X_{2}\right)$ into $\mathscr{D}_{\chi^{*} V}^{\prime}\left(X_{1}\right)$ if $V \cap N_{\chi}=\emptyset$. Here, for each closed conic subset $V \subset T^{*} X_{2}$, the set $\mathscr{D}_{V}^{\prime}\left(X_{2}\right)$ is a linear subspace of the distribution space $\mathscr{D}^{\prime}\left(X_{2}\right)$ which is defined as $\mathscr{D}_{V}^{\prime}\left(X_{2}\right)=\{u \in$ 
$\left.\mathscr{D}^{\prime}\left(X_{2}\right): \mathrm{WF}(u) \subset V\right\}$. As we will discuss below, there is a notion of convergence in $\mathscr{D}_{V}^{\prime}\left(X_{2}\right)$ with respect to which $\mathscr{D}_{V}^{\prime}\left(X_{2}\right)$ is a closed subset of $\mathscr{D}^{\prime}\left(X_{2}\right)$ (cf. also Def. 8.2.2 in [25]). This notion of convergence is sometimes referred to as the "Hörmander pseudotopology" of $\mathscr{D}_{V}^{\prime}\left(X_{2}\right)$ and it is this sense in which $\chi^{*}$ is continuous, $\mathscr{D}_{\chi^{*} V}^{\prime}\left(X_{1}\right)$ being analogously defined.

Convergence in the Hörmander pseudo-topology may be defined as follows. Suppose $u_{r}$ is a sequence in $\mathscr{D}_{V}^{\prime}(X)$ and $u \in \mathscr{D}_{V}^{\prime}(X)$. Then $u_{r} \rightarrow u$ in $\mathscr{D}_{V}^{\prime}(X)$ if the two following conditions hold:

(i) $u_{r} \rightarrow u$ weakly in $\mathscr{D}^{\prime}(X)$ (i.e., $u_{r}(f) \rightarrow u(f)$ for each test function $f$ )

(ii) for all $(x, k) \in \dot{T}^{*} X \backslash V$, there exists $(\mathcal{O}, \phi)$ obeying condition (A) above, $\chi \in C_{0}^{\infty}(\mathcal{O})$ with $\chi(x) \neq 0$ and a neighbourhood $E$ of $k$ in $T_{x}^{*} X$ obeying

$$
\left[\phi^{*}(\phi(\operatorname{supp} \chi) \times E)\right] \cap V=\emptyset
$$

and such that the quantities

$$
\sup _{\lambda \in \mathbb{R}^{+}} \sup _{\ell \in E} \lambda^{N}\left|u_{r}\left(\chi e^{i \lambda\langle\ell, \phi\rangle}\right)\right|
$$

are uniformly bounded in $r$ for each $N=1,2, \ldots$

Any distribution $u \in \mathscr{D}^{\prime}(X)$ may be arbitrarily well approximated in $\mathscr{D}_{V}^{\prime}(X)$, for any $V \supset \mathrm{WF}(u)$, by a sequence of test functions $u_{r} \in \mathscr{D}(X)$; it is this which permits the definition of pull-backs to be extended to distributions by continuity from the definition for functions.

The notion of Hörmander pseudo-topology is easily extended to distributions taking values in a Banach space $B$ simply by replacing modulus signs with Banach norms where appropriate, and denoting by $\mathscr{D}_{V}^{\prime}(X, B)$ the set of distributions in $\mathscr{D}^{\prime}(X, B)$ whose wavefront sets are contained in $V$.

It is useful to have some simpler sufficient conditions for convergence in $\mathcal{D}_{V}^{\prime}(X, B)$. It is not hard to show that, for example, if $u_{r}$ is a sequence converging weakly to $u$ in $\mathscr{D}^{\prime}(X, B)$ and $\left\|u_{r}(f)\right\| \leq\|v(f)\|$ for all $r$ and some $v \in \mathscr{D}^{\prime}(X, B)$ then $u_{r} \rightarrow u$ in $\mathscr{D}_{\mathrm{WF}(v)}^{\prime}(X)$. The following is a slight elaboration of this observation.

Proposition 3.4 Let $X$ and $Y$ be $C^{\infty}$-manifolds and $\left(B_{i},\|\cdot\|_{i}\right)$ be Banach spaces $(i=$ $1,2)$. Suppose that $u_{r}$ is a sequence in $\mathscr{D}^{\prime}\left(X \times Y, B_{1}\right)$ converging weakly to $u$. Suppose further that there exists $v \in \mathscr{D}^{\prime}\left(X \times Y, B_{2}\right)$ such that

$$
\left\|u_{r}(f, g)\right\|_{1} \leq\|v(f, g)\|_{2}
$$

for all $f \in \mathscr{D}(X)$ and $g \in \mathscr{D}(Y)$. Then $u_{r} \rightarrow u$ in the Hörmander pseudo-topology on $\mathscr{D}_{\mathrm{WF}(v)}^{\prime}(X \times Y)$.

Remark: The result continues to hold if Eq. (3.2) is generalised to

$$
\left\|u_{r}(f, g)\right\|_{1} \leq \sum_{i=1}^{n}\left\|v_{i}(f, g)\right\|_{i}
$$


for Banach spaces $\left(B_{i},\|\cdot\|_{i}\right)$ and distributions $v_{i} \in \mathcal{D}^{\prime}\left(X \times Y, B_{i}\right),(i=1, \ldots, n)$ by applying the proposition to $v=v_{1} \oplus v_{2} \oplus \cdots \oplus v_{n} \in \mathcal{D}^{\prime}\left(X \times Y, \bigoplus_{i=1}^{n} B_{i}\right)$.

Proof: Suppose $\left(x_{0}, y_{0} ; k_{0}, l_{0}\right)$ is a regular directed point for $v$. Let $\left(\mathcal{O}_{X}, \phi_{X}\right)$ and $\left(\mathcal{O}_{Y}, \phi_{Y}\right)$ obey condition (A) above for points $x_{0} \in X$ and $y_{0} \in Y$, and define

$$
\phi(x, y)=\phi_{X}(x) \oplus \phi_{Y}(y) \in T_{x_{0}} X \oplus T_{y_{0}} Y \cong T_{\left(x_{0}, y_{0}\right)}(X \times Y) .
$$

Then $\left(\mathcal{O}_{X} \times \mathcal{O}_{Y}, \phi\right)$ obeys condition $(\mathrm{A})$ for $\left(x_{0}, y_{0}\right)$; furthermore, by continuity of the pull-back $\phi^{*}$ and the fact that $\mathrm{WF}(v)$ is closed, there exists open $\mathcal{O} \subset \mathcal{O}_{X} \times \mathcal{O}_{Y}$ and a neighbourhood $G$ of $\left(k_{0}, l_{0}\right)$ in $T_{\left(x_{0}, y_{0}\right)}^{*} X \times Y$ such that

$$
\left[\phi^{*}(\phi(\mathcal{O}) \times G)\right] \cap \mathrm{WF}(v)=\emptyset .
$$

Since $\left(x_{0}, y_{0} ; k_{0}, l_{0}\right)$ is regular directed for $v$, there exists $\chi \in C_{0}^{\infty}(\mathcal{O})$ with $\chi\left(x_{0}, y_{0}\right) \neq 0$ and a neighbourhood $E$ of $\left(k_{0}, l_{0}\right)$, contained (without loss of generality) in $G$ such that

$$
\lambda^{N} \sup _{(k, l) \in E}\left\|v\left(\chi e^{i \lambda\langle(k, l), \phi\rangle}\right)\right\|_{2} \rightarrow 0 \quad \text { as } \lambda \rightarrow+\infty
$$

for each $N$. Choose smooth functions $\eta_{X}$ and $\eta_{Y}$ such that $\eta(x, y)=\eta_{X}(x) \eta_{Y}(y)$ is compactly supported in the interior of $\operatorname{supp} \chi$, with $\eta\left(x_{0}, y_{0}\right) \neq 0$. Then

$$
\psi(x, y)=\left\{\begin{array}{cl}
\eta(x, y) / \chi(x, y) & (x, y) \in \operatorname{supp} \eta \\
0 & \text { otherwise }
\end{array}\right.
$$

is smooth and compactly supported and Eq. (3.3.3) continues to hold if $\chi$ is replaced by $\psi \chi=\eta$, thereby yielding

$$
\lambda^{N} \sup _{(k, l) \in E}\left\|v\left(\eta_{X} e^{i \lambda\left\langle k, \phi_{X}\right\rangle}, \eta_{Y} e^{i \lambda\left\langle l, \phi_{Y}\right\rangle}\right)\right\|_{2} \rightarrow 0 \quad \text { as } \lambda \rightarrow+\infty
$$

for each $N$. Together with Eq. (3.2) this immediately implies that $\left(x_{0}, y_{0} ; k_{0}, l_{0}\right)$ is a regular directed point for each $u_{r}$ and $u$. It follows that $u_{r}$ and $u$ belong to $\mathscr{D}_{\mathrm{WF}(v)}^{\prime}\left(X, B_{1}\right)$. Furthermore,

$$
\sup _{\lambda \in \mathbb{R}^{+}} \sup _{(k, l) \in E} \lambda^{N}\left\|u_{r}\left(\chi e^{i \lambda\langle\ell, \phi\rangle}\right)\right\|_{1} \leq \sup _{\lambda \in \mathbb{R}^{+}} \sup _{(k, l) \in E} \lambda^{N}\left\|v\left(\eta_{X} e^{i \lambda\left\langle k, \phi_{X}\right\rangle}, \eta_{Y} e^{i \lambda\left\langle l, \phi_{Y}\right\rangle}\right)\right\|_{2},
$$

the right-hand side of which is easily seen to be finite. This provides the required uniform bound to ensure that $u_{r} \rightarrow u$ in $\mathscr{D}_{\mathrm{WF}(v)}^{\prime}\left(X, B_{1}\right)$.

\section{Quantum fields on static backgrounds}

We will now describe how the structural assumptions made in Sec. 2 may be justified for the case of real scalar field theory on a globally hyperbolic static spacetime $(M, \boldsymbol{g})$ with compact spatial sections. The assumptions to be checked are:

- the existence of a $C^{*}$-dynamical system along with a suitable sub-*-algebra $\mathcal{A}^{\infty}$ and a generating linear space $\mathcal{W}^{\infty}$ which is stable under convolutions; 
- the identification of a set of states $\mathcal{S}$ closed under finite convex combinations and operations induced by elements of the algebra $\mathcal{U}_{\text {alg }}^{\infty}$ constructed from $\mathcal{A}^{\infty}$;

- the existence of an energy density [defined for every state in $\mathcal{S}$ ] whose spatial integral generates the dynamics;

- the existence of states satisfying a suitable static QWEI.

Each assumption will be treated in turn in the following subsections. Most details are postponed to the Appendix.

It is worth mentioning that we will also prove a converse to Thm. 2.5)(a) for the free scalar field: namely, we will show in Thm. 4.8 that a non-degenerate ground state with mass gap and vanishing one-point functions is necessarily quiescent.

\subsection{The dynamical system}

We begin by reviewing the quantisation of the real scalar field on a globally hyperbolic static spacetime $(M, \boldsymbol{g})$. Such a spacetime is diffeomorphic to $\mathbb{R} \times \Sigma$ with line element

$$
d s^{2}=g_{a b} d x^{a} d x^{b}=g_{00} d t^{2}-h_{i j} d x^{i} d x^{j},
$$

where $\boldsymbol{h}$ is a (positive definite) Riemannian metric and $g_{00}$ is a smooth strictly positive function on $\Sigma$. As before, we will assume that $\Sigma$ is $s$-dimensional and compact; the preferred measure on $\Sigma$ is $d \mu(\underline{x})=\sqrt{h} d^{s} \underline{x}$, where $h=\operatorname{det} \boldsymbol{h}$. The Killing vector $\partial / \partial t$ will be denoted $\xi$. We will also introduce an orthonormal frame $e_{\mu}^{a}(\mu=0, \ldots, s)$ with $e_{0}^{a}=g_{00}^{-1 / 2} \xi^{a}$.

The Klein-Gordon equation on $(M, \boldsymbol{g})$ is

$$
\left(g^{a b} \nabla_{a} \nabla_{b}+m^{2}\right) \varphi=0,
$$

for which the corresponding classical stress-energy tensor is

$$
T_{a b}=\nabla_{a} \varphi \nabla_{b} \varphi-\frac{1}{2} g_{a b} g^{c d} \nabla_{c} \varphi \nabla_{d} \varphi+\frac{1}{2} m^{2} g_{a b} \varphi^{2} .
$$

Integrating over a surface of constant 'time' $\{t\} \times \Sigma(t \in \mathbb{R})$ we obtain the classical energy

$$
H=\int_{\Sigma} T_{a b}(t, \underline{x}) n^{a} \xi^{b} d \mu(\underline{x})
$$

where $n^{a}=g_{00}^{-1 / 2} \xi^{a}=e_{0}^{a}$ is the future-pointing unit normal to $\Sigma$; this is conserved by virtue of the Klein-Gordon equation and Gauss' theorem. In addition, the classical energy density seen by an observer with velocity $e_{0}^{a}$ is

$$
e_{0}^{a} e_{0}^{b} T_{a b}=\frac{1}{2}\left(m^{2} \varphi^{2}+\sum_{\mu=0}^{s}\left(e_{\mu}^{a} \nabla_{a} \varphi\right)^{2}\right) .
$$


The quantisation of this system proceeds as follows (cf. [9]): first, let $(S, \sigma)$ be the symplectic space of smooth real-valued Klein-Gordon solutions where the symplectic form is given by

$$
\sigma(u, v)=\int_{\Sigma} d \mu(\underline{x})\left(u e_{0}^{a} \nabla_{a} v-v e_{0}^{a} \nabla_{a} u\right) .
$$

The CCR-algebra (or Weyl algebra) $\mathfrak{A}[S, \sigma]$ over $(S, \sigma)$ is the (unique up to $C^{*}$-isomorphism) unital $C^{*}$-algebra generated over $\mathbb{C}$ by unitary elements $\mathrm{W}(u)(u \in S)$ with $\mathrm{W}(0)=\mathbf{1}$ subject to the Weyl relations

$$
\mathbf{W}(u) \mathbf{W}(v)=e^{-i \sigma(u, v) / 2} \mathbf{W}(u+v), \quad u, v \in S .
$$

For each open relatively compact $O \subset M$, let $\mathfrak{A}(O)$ be the sub- $C^{*}$-algebra of $\mathfrak{A}[S, \sigma]$ generated by elements of the form $\mathrm{W}(E f)$ where $f \in C_{0}^{\infty}(O ; \mathbb{R})$ and $E: C_{0}^{\infty}(M) \rightarrow$ $C^{\infty}(M)$ is the advanced-minus-retarded fundamental solution for the Klein-Gordon equation. Then $O \mapsto \mathfrak{A}(O)$ is an isotonous net of $C^{*}$-algebras which is also local in the sense that $A_{1} A_{2}=A_{2} A_{1}$ holds for all $A_{1} \in \mathfrak{A}\left(O_{1}\right)$ and $A_{2} \in \mathfrak{A}\left(O_{2}\right)$ whenever the regions $O_{1}$ and $\mathrm{O}_{2}$ in $M$ cannot be connected by any causal curve. Hence, the net $O \mapsto \mathfrak{A}(O)$ is the essential building block of a local quantum field theory on the curved spacetime $(M, \boldsymbol{g})$ (cf. [23, 48]).

Since the time translations $\tau_{t}\left(t^{\prime}, \underline{x}\right)=\left(t+t^{\prime}, \underline{x}\right)$ induce a symplectomorphism of $(S, \sigma)$ there is a 1-parameter group of $*$-automorphisms on $\mathfrak{A}[S, \sigma]$ given by

$$
\tilde{\alpha}_{t}(\mathrm{~W}(u))=\mathrm{W}\left(\tau_{t *} u\right), \quad u \in S, t \in \mathbb{R},
$$

where $\tau_{t *} u=u \circ \tau_{t}^{-1}$ is the push-forward. ${ }^{7}$ The $C^{*}$-algebraic net $O \mapsto \mathfrak{A}(O)$ then has the covariance property [9]

$$
\tilde{\alpha}_{t}(\mathfrak{A}(O))=\mathfrak{A}\left(\tau_{t}(O)\right) .
$$

However, $\left\{\tilde{\alpha}_{t}\right\}_{t \in \mathbb{R}}$ is not strongly continuous (because $\|\mathrm{W}(u)-\mathbf{W}(v)\|=2$ for all $u \neq v$ ). This obstacle can be circumvented as we shall explain, but let us first give a brief description of how we proceed. We will, in the following subsection, work in GNS-representations of quasifree Hadamard states which may be regarded as states in quantum field theory on curved spacetimes whose short-distance behaviour is close to that of vacuum states or thermal equilibrium states. We shall denote by $\mathcal{S}_{\mathrm{qH}}$ the set of all quasifree Hadamard states on $\mathfrak{A}[S, \sigma]$, and starting from this class we shall define the underlying $C^{*}$-dynamical system, the sub-*-algebra $\mathcal{A}^{\infty}$ and the set of states $\mathcal{S}$. In the remainder of this subsection we will discuss the $C^{*}$-dynamical system, and the algebra $\mathcal{A}^{\infty}$.

Consider a state $\omega$ on $\mathfrak{A}[S, \sigma]$ and let $\left(\mathcal{H}_{\omega}, \pi_{\omega}, \Omega_{\omega}\right)$ be its GNS-representation. Then we call such a state weakly covariant if there exists on the von Neumann algebra $\mathcal{M}_{\omega}=$ $\pi_{\omega}(\mathfrak{A}[S, \sigma])^{\prime \prime}$ a one-parameter group $\left\{\alpha_{t}^{(\omega)}\right\}_{t \in \mathbb{R}}$ of automorphisms (leaving $\mathcal{M}_{\omega}$ invariant) so that

$$
\alpha_{t}^{(\omega)} \circ \pi_{\omega}(\mathrm{A})=\pi_{\omega} \circ \tilde{\alpha}_{t}(\mathrm{~A}), \quad t \in \mathbb{R}, \mathrm{A} \in \mathfrak{A}[S, \sigma] .
$$

A special case is a covariant state $\omega$ where $\alpha_{t}^{(\omega)}(A)=V_{t}^{(\omega)} A V_{t}^{(\omega) *}$ with a strongly continuous unitary group $\left\{V_{t}^{(\omega)}\right\}_{t \in \mathbb{R}}$ on $\mathcal{H}_{\omega}$. Then $\left(\mathcal{M}_{\omega},\left\{\alpha_{t}^{(\omega)}\right\}_{t \in \mathbb{R}}\right)$ is a $W^{*}$-dynamical system

\footnotetext{
${ }^{7}$ The push-forward $\tau_{t *}$ and pull-back $\tau_{t}^{*}$ are related by $\tau_{-t}^{*} u=u \circ\left(\tau_{-t}\right)=u \circ \tau_{t}^{-1}=\tau_{t *} u$.
} 
(cf. [6]), but we need to pass to a $C^{*}$-dynamical system whose definition we now describe, following the strategy outlined in the Introduction. Consider the operators $\alpha_{f} A$ defined by (1.4) with $\alpha_{t} \equiv \alpha_{t}^{(\omega)}$ for $f \in C_{0}^{\infty}(\mathbb{R})$ and $A \in \pi_{\omega}(\mathfrak{A}[S, \sigma])$ where the integral is understood in the weak topology, so $\alpha_{f} A \in \mathcal{M}_{\omega}$. It is straightforward to check that $\left\|\alpha_{f} A\right\| \leq\|f\|_{L^{1}}\|A\|$ and $\alpha_{t}^{(\omega)}\left(\alpha_{f} A\right)=\alpha_{f(.-t)} A$, so that $\left\|\alpha_{t}^{(\omega)}\left(\alpha_{f} A\right)-\alpha_{f} A\right\| \rightarrow 0$ for $t \rightarrow 0$. Now we define $\mathcal{A}_{\omega} \subset \mathcal{M}_{\omega}$ as the $C^{*}$-closure of the $*$-algebra generated by all these $\alpha_{f} A$; then $\left(\mathcal{A}_{\omega},\left\{\alpha_{t}^{(\omega)}\right\}_{t \in \mathbb{R}}\right)$ is a $C^{*}$-dynamical system (where $\alpha_{t}^{(\omega)}$ should here strictly be read as $\left.\alpha_{t}^{(\omega)} \uparrow \mathcal{A}_{\omega}\right)$. Now this dynamical system depends on the chosen state $\omega$, but for our discussion, this dependence is spurious, as we shall explain.

As mentioned above, we denote by $\mathcal{S}_{\mathrm{qH}}$ the set of quasifree Hadamard states on $\mathfrak{A}[S, \sigma]$ (see next subsection for a definition). At the present stage of discussion it is important to know that, whenever $\omega_{1}$ and $\omega_{2}$ are contained in $\mathcal{S}_{\mathrm{qH}}$, then $\omega_{1}$ and $\omega_{2}$ are quasi-equivalent 43. (because of spatial compactness of the underlying spacetime). This means in the notation just introduced that there are von Neumann-algebra isomorphisms $\beta_{21}: \mathcal{M}_{\omega_{1}} \rightarrow$ $\mathcal{M}_{\omega_{2}}$ and $\beta_{12}: \mathcal{M}_{\omega_{2}} \rightarrow \mathcal{M}_{\omega_{1}}$ such that $\beta_{21} \circ \pi_{\omega_{1}}=\pi_{\omega_{2}}$ and $\beta_{12} \circ \pi_{\omega_{2}}=\pi_{\omega_{1}}$. We recall also that all quasifree states on $\mathfrak{A}[S, \sigma]$ are faithful, and so are their GNS-representations. Thus $\beta_{21}=\beta_{12}^{-1}$. Moreover, if any state $\omega_{1} \in \mathcal{S}_{\mathrm{qH}}$ turns out to be covariant, then all $\omega_{2} \in \mathcal{S}_{\mathrm{qH}}$ are weakly covariant, too, with

$$
\alpha_{t}^{\left(\omega_{2}\right)}=\beta_{21} \circ \alpha_{t}^{\left(\omega_{1}\right)} \circ \beta_{12} .
$$

It is also straightforward to check that $\mathcal{A}_{\omega_{2}}=\beta_{21}\left(\mathcal{A}_{\omega_{1}}\right)$. In this sense, the $C^{*}$-dynamical system $\left(\mathcal{A}_{\omega},\left\{\alpha_{t}^{(\omega)}\right\}_{t \in \mathbb{R}}\right)$ is independent of the chosen state $\omega \in \mathcal{S}_{\mathrm{qH}}$ once it is known that there exist quasifree Hadamard states for $\mathfrak{A}[S, \sigma]$ which are weakly covariant. This, however, can also be concluded from the fact that each pair of states in $\mathcal{S}_{\mathrm{qH}}$ is quasiequivalent. To see this one simply notes that for each $\omega \in \mathcal{S}_{\mathrm{qH}}$ the time-shifted state $\omega \circ \alpha_{t}$ is again in $\mathcal{S}_{\mathrm{qH}}$ - this is a consequence of the fact that the wave-front set of the two-point function (cf. Sec. 4.2) of a quasifree Hadamard state is left invariant under the isometries $\tau_{t}$. Thus, since $\pi_{\omega} \circ \tilde{\alpha}_{t}$ and $\pi_{\omega \circ \tilde{\alpha}_{t}}$ are canonically unitarily equivalent by the uniqueness of the GNS-representation, there is for each $t$ a von Neumann algebraic isomorphism $\alpha_{t}^{(\omega)}: \mathcal{M}_{\omega} \rightarrow \mathcal{M}_{\omega}$ with the covariance property (4.2). Hence, starting from the class of states $\mathcal{S}_{\mathrm{qH}}$, we see that there is (up to isomorphism) a unique $C^{*}$-dynamical system associated with it.

Now, for $\omega \in \mathcal{S}_{\mathrm{qH}}$ we define the sub-vector space $\mathcal{W}_{\omega}^{\infty}$ of $\mathcal{A}_{\omega}$ as the vector space generated by all $\alpha_{f} \pi_{\omega}(\mathrm{W}(u))$ where $f \in C_{0}^{\infty}(\mathbb{R})$ and $u \in S$, and denote by $\mathcal{A}_{\omega}^{\infty}$ the *algebra generated by $\mathcal{W}_{\omega}^{\infty}$. As a consequence of the Weyl-relations, it is straightforward to check that $\mathcal{W}_{\omega}^{\infty}$ is norm-dense in $\mathcal{A}_{\omega}$ and stable under taking adjoints; it is stable under convolution with test-functions with respect to $\alpha_{t}$ by its very definition. Moreover, one may easily check that $\mathcal{A}_{\omega_{2}}^{\infty}=\beta_{21}\left(\mathcal{A}_{\omega_{1}}^{\infty}\right)$ for all $\omega_{1}, \omega_{2} \in \mathcal{S}_{\mathrm{qH}}$. The vector space $\mathcal{W}_{\omega}^{\infty}$ and algebra $\mathcal{A}_{\omega}^{\infty}$ are uniquely associated with $\mathcal{S}_{\mathrm{qH}}$ up to isomorphism, just as the $C^{*}$-dynamical system was. In this light, we shall henceforth adopt the following conventions:

- we choose an arbitrary, quasifree Hadamard state $\omega_{0}$ and keep it fixed,

- $\quad$ we denote by $\left(\mathcal{A},\left\{\alpha_{t}\right\}_{t \in \mathbb{R}}\right), \mathcal{W}^{\infty}$ and $\mathcal{A}^{\infty}$ the $C^{*}$-dynamical system, dense subspace and $*$-algebra associated with $\omega_{0}$ as just described. 
In applying the abstract results of Sec. 2, we will take $\left(\mathcal{A},\left\{\alpha_{t}\right\}_{t \in \mathbb{R}}\right)$ to be the $C^{*}$-dynamical system of interest.

The results of Sec. 2 then assert the existence of passive states $\omega^{p}$ on $\mathcal{A}$ as a consequence of suitable forms of static quantum weak energy inequalities (which will be established in the following sections) and the question might arise under which conditions the states $\omega^{p}$ can be interpreted as passive states on the original Weyl algebra $\mathfrak{A}[S, \sigma]$. The following lemma shows that these states always induce states on $\mathfrak{A}[S, \sigma]$ (recalling that a passive state is always invariant under the time-evolution).

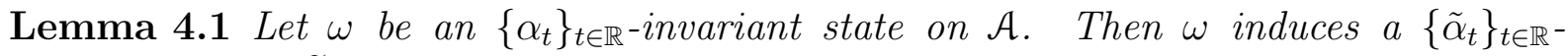
invariant state $\omega^{\mathfrak{A}}$ on $\mathfrak{A}[S, \sigma]$.

Proof. For all $f \in C_{0}^{\infty}(\mathbb{R})$ and all $A=\pi_{\omega_{0}}(\mathrm{~A}) \in \pi_{\omega_{0}}(\mathfrak{A}[S, \sigma])$ the estimate

$$
\left|\omega\left(\alpha_{f} A\right)\right| \leq\|f\|_{L^{1}}\|A\|
$$

entails that, for fixed $A, f \mapsto \omega\left(\alpha_{f} A\right)$ extends to a continuous linear functional on $L^{1}(\mathbb{R})$. Consequently, there exists a function $L_{A} \in L^{\infty}(\mathbb{R})$ so that

$$
\omega\left(\alpha_{f} A\right)=\int d t L_{A}(t) f(t), \quad f \in L^{1}(\mathbb{R}) .
$$

Now as $\omega$ is $\left\{\alpha_{t}\right\}_{t \in \mathbb{R}}$-invariant, it follows easily that $L_{A}$ must be constant (almost everywhere). Let us denote this constant by $\bar{\omega}(A)$, then it holds that

$$
\omega\left(\alpha_{f} A\right)=\bar{\omega}(A) \int d t f(t), \quad f \in L^{1}(\mathbb{R}),
$$

showing that $\bar{\omega}(A)=\omega\left(\alpha_{f} A\right)$ whenever $\int d t f(t)=1$. The assignment $A \mapsto \bar{\omega}(A)$ is obviously linear and we need to show that it fulfills state-positivity. Let $B=A^{*} A$ be a positive element in $\pi_{\omega_{0}}(\mathfrak{A}[S, \sigma])$. All we need to demonstrate is the existence of some $f \in L^{1}(\mathbb{R})$ with $\int d t f(t)=1$ so that $\alpha_{f} B$ is a positive element in $\mathcal{A}$ (whereupon $\left.\bar{\omega}(B)=\omega\left(\alpha_{f} B\right) \geq 0\right)$. Choosing any $f \geq 0$ in $L^{1}(\mathbb{R})$ with $\int d t f(t)=1$, it is clear that $\alpha_{f} B$ is a positive element in $\mathcal{B}\left(\mathcal{H}_{\omega_{0}}\right)$. But since $\mathcal{A}$ inherits the $*$-operation and $C^{*}$-norm of $\mathcal{B}\left(\mathcal{H}_{\omega_{0}}\right)$, it follows that $\alpha_{f} B$ is also a positive element in $\mathcal{A}$ (cf. Lemma 2.2.9 in [6]). Moreover, $\bar{\omega}\left(\alpha_{t}(A)\right)=\omega\left(\alpha_{f}\left(\alpha_{t} A\right)\right)=\omega\left(\alpha_{t}\left(\alpha_{f} A\right)\right)=\omega\left(\alpha_{f} A\right)=\bar{\omega}(A)$ so $\bar{\omega}$ is an $\left\{\alpha_{t}\right\}_{t \in \mathbb{R}^{-}}$ invariant state on $\pi_{\omega_{0}}(\mathfrak{A}[S, \sigma])$. Thus $\omega^{\mathfrak{A}}=\bar{\omega} \circ \pi_{\omega_{0}}$ is an $\left\{\tilde{\alpha}_{t}\right\}_{t \in \mathbb{R}^{-}}$-invariant state on $\mathfrak{A}[S, \sigma]$.

However, it should be noted that one has no information regarding the continuity of $\omega^{\mathfrak{A}}$ with respect to the time-evolution, in other words, there is no reason why the functions $t \mapsto \omega^{\mathfrak{A}}\left(\mathrm{A} \tilde{\alpha}_{t}(\mathrm{~B})\right), \mathrm{A}, \mathrm{B} \in \mathfrak{A}[S, \sigma]$, should be continuous, and therefore it is unclear if $\omega^{\mathfrak{A}}$ is passive (in a $W^{*}$-sense) on $\mathfrak{A}[S, \sigma]$ if $\omega$ is a passive state on $\mathcal{A}$. This can be concluded if $\omega$ fulfills further regularity conditions. A sufficient condition to that effect is that $\omega=\omega^{p}$ be a normal state on $\mathcal{M}_{\omega_{0}}$, and we have seen in Cor. 2.7 that a certain energy-compactness condition ensures this normality.

We should also like to point out that one can generalize the notion of $n$-point correlation functions so that it is applicable to states on $\mathcal{A}$ in the sense that sufficently regular 
states (" $C^{\infty}$-regular states") on $\mathcal{A}$ possess $n$-point correlation functions for all $n \in \mathbb{N}$, inducing states on the algebra of abstract Klein-Gordon field operators. These matters will be discussed in Appendix A.5. The result of this discussion again shows that there is hardly any difference in working with $\mathfrak{A}[S, \sigma]$ or $\mathcal{A}$ as long as "sufficiently regular" states are considered, and thus our passing from $\mathfrak{A}[S, \sigma]$ to $\mathcal{A}$ can rightfully be regarded as made purely for technical convenience.

\subsection{The state space $\mathcal{S}$}

The states to be considered are drawn from the class of Hadamard states on $\mathfrak{A}[S, \sigma]$, for which the renormalised energy density may be defined by point-splitting. They are defined as follows. Suppose a state $\omega$ is sufficiently regular that the function $(s, t) \mapsto$ $\omega(\mathrm{W}(s E f) \mathrm{W}(t E g))$ is twice continuously differentiable for each pair of real-valued test functions $f, g$ and that, moreover, the two-point function $w_{2}^{(\omega)}$ defined by

$$
w_{2}^{(\omega)}(f, g)=-\left.\frac{\partial^{2}}{\partial s \partial t} \omega(\mathrm{W}(s E f) \mathrm{W}(t E g))\right|_{s, t=0}, \quad f, g \in \mathscr{D}(M ; \mathbb{R})
$$

extends (by complex linearity in its arguments) to a distribution in $\mathscr{D}^{\prime}(M \times M)$. Then

$\omega$ is said to be Hadamard if the corresponding 2-point correlation function $w_{2}^{(\omega)}$ takes the so-called Hadamard form [27], which completely fixes $w_{2}^{(\omega)}$ modulo smooth terms; in particular, the difference between any two Hadamard two-point functions is smooth. In 34, Radzikowski showed that this condition could be replaced by the requirement that the wave-front set of the two-point function should satisfy

$$
\mathrm{WF}\left(w_{2}^{(\omega)}\right)=\left\{\left(x, k ; x^{\prime},-k^{\prime}\right) \in \dot{T}^{*}(M \times M):(x, k) \sim\left(x^{\prime}, k^{\prime}\right), k \in \mathcal{N}_{x}^{+}\right\},
$$

where $\mathcal{N}_{x}^{+}$is the cone of (non-zero) future-pointing null covectors at $x$ and $(x, k) \sim\left(x^{\prime}, k^{\prime}\right)$ if there is a null geodesic connecting $x$ and $x^{\prime}$ to which $k$ and $k^{\prime}$ are cotangent at $x$ and $x^{\prime}$ respectively, with $k^{\prime}$ being the parallel transport of $k$. [In the case $x=x^{\prime}$, we require $k=k^{\prime}$.] For future reference we will also use $\mathcal{N}_{x}^{-}$to denote the cone of non-zero pastpointing null covectors at $x$ and $\mathcal{N}^{ \pm}=\bigcup_{x \in M} \mathcal{N}_{x}^{ \pm}$for the future and past null cones in $T^{*} M$.

Radzikowski's criterion has been simplified recently by Strohmaier, Wollenberg and Verch [40], who consider Hilbert-space valued distributions induced by the field operators. Their characterisation is essentially the following.

Theorem 4.2 A state $\omega$ on $\mathfrak{A}[S, \sigma]$ is Hadamard if and only if the following conditions hold in some GNS representation [not necessarily that induced by $\omega](\mathcal{H}, \pi, \Omega)$ of $\mathfrak{A}[S, \sigma]$ :

a). $\omega$ is represented by a vector $\psi \in \mathcal{H}$, i.e., $\omega(A)=\langle\psi, \pi(A) \psi\rangle$ for all $A \in \mathfrak{A}[S, \sigma]$;

b). the function $t \mapsto \pi(\mathrm{W}(t E f)) \psi$ is differentiable for all $f \in \mathscr{D}(M ; \mathbb{R})$;

c). the $\mathcal{H}$-valued functional $f \mapsto \Phi(f) \psi:=-i d /\left.d t \pi(\mathrm{W}(t E f)) \psi\right|_{t=0}$ extends by complexlinearity to a Hilbert-space valued distribution $\Phi(\cdot) \psi \in \mathscr{D}^{\prime}(M, \mathcal{H})$ obeying

$$
\mathrm{WF}(\Phi(\cdot) \psi) \subset \mathcal{N}^{-}
$$


Remark: Condition a) may always be satisfied by using the GNS representation induced by $\omega$, but it is convenient to allow for other representations. Note that the assignment $f \mapsto$ $\Phi(f) \psi$ defines the field operator $\Phi(f)$ in the GNS-Hilbert-space representation $(\mathcal{H}, \pi, \Omega)$ on the domain $D(\Phi(f))$ of all $\psi \in \mathcal{H}$ for which $-i d /\left.d t \pi(\mathrm{W}(t E f)) \psi\right|_{t=0}$ exists as strong limit in $\mathcal{H}$.

Now let $\omega$ be any quasifree Hadamard state state on $\mathfrak{A}[S, \sigma]$, and denote by $\left(\mathcal{H}_{\omega}, \pi_{\omega}, \Omega_{\omega}\right)$ the corresponding GNS-representation, and by $\Phi_{\omega}(f)=-i d /\left.d t \pi_{\omega}(\mathrm{W}(t E f))\right|_{t=0}$ the field operators, defined on a dense domain $D\left(\Phi_{\omega}(f)\right) \subset \mathcal{H}_{\omega}$ for $f \in \mathscr{D}(M)$. We define $\operatorname{Had}(\omega)$ as the set of vectors $\psi \in \bigcap_{f \in \mathscr{D}(M)} D\left(\Phi_{\omega}(f)\right)$ having the property that $\Phi_{\omega}(\cdot) \psi$ belongs to $\mathscr{D}^{\prime}\left(M, \mathcal{H}_{\omega}\right)$ and obeys the Hadamard condition (4.4). For every quasifree Hadamard state $\omega$, the vectors $\psi \in \operatorname{Had}(\omega)$ induce states and, more generally, continuous linear functionals on the $C^{*}$-algebra $\mathcal{A}=\mathcal{A}_{\omega_{0}}$ of our dynamical system. Namely, let $\beta_{\omega \omega_{0}}: \mathcal{M}_{\omega_{0}} \rightarrow \mathcal{M}_{\omega}$ be the von Neumann-algebra isomorphism with $\beta_{\omega \omega_{0}} \circ \pi_{\omega_{0}}=\pi_{\omega}$, then

$$
\omega^{[\psi]}(A)=\left\langle\psi, \beta_{\omega \omega_{0}}(A) \psi\right\rangle, \quad A \in \mathcal{A}_{\omega_{0}},
$$

is a state on $\mathcal{A}=\mathcal{A}_{\omega_{0}}$. We now define the state space $\mathcal{S}$ as the set of finite convex combinations of states induced by vectors in $\operatorname{Had}(\omega), \omega \in \mathcal{S}_{\mathrm{qH}}$. In other words, a state $\tilde{\omega}$ on $\mathcal{A}$ is contained in $\mathcal{S}$ iff there are finitely many quasifree Hadamard states $\omega_{i} \in \mathcal{S}_{\mathrm{qH}}$ $(i=1, \ldots, N)$ together with unit vectors $\psi_{i} \in \operatorname{Had}\left(\omega_{i}\right)$ and $\lambda_{i}>0, \sum_{i=1}^{N} \lambda_{i}=1$, such that

$$
\tilde{\omega}(A)=\sum_{i=1}^{N} \lambda_{i} \omega_{i}^{\left[\psi_{i}\right]}(A), \quad A \in \mathcal{A} .
$$

Theorem 4.2 guarantees that all the states in $\mathcal{S}$ are Hadamard states. The state space $\mathcal{S}$ has the following properties, as will be proved in Appendix A.1:

Proposition $4.3 \mathcal{S}_{\mathrm{qH}} \subset \mathcal{S}$; and $\mathcal{S}$ is closed under finite convex combinations and operations in $\mathcal{U}_{\mathrm{alg}}^{\infty}$.

\subsection{The energy density}

Let $\omega \in \mathcal{S}_{\mathrm{qH}}$ and define $\mathcal{F}_{\omega}$ to consist of all linear functionals (not, in general, states) $\ell$ on $\mathcal{M}_{\omega_{0}} \supset \mathcal{A}$ given by

$$
\ell(B)=\left\langle\psi, \beta_{\omega \omega_{0}}(B) \varphi\right\rangle, \quad B \in \mathcal{M}_{\omega_{0}},
$$

for some $\psi, \varphi \in \operatorname{Had}(\omega)$. We also denote by $\mathcal{F}$ the set of all linear combinations of finitely many functionals $\ell_{i} \in \mathcal{F}_{\omega_{i}}, \omega_{i} \in \mathcal{S}_{\mathrm{qH}}$ and-as in Sec. 2-use $\mathcal{V}$ to denote the vector space of functionals on $\mathcal{A}$ generated (as in Eq. (2.1)) by $\mathcal{S}$ and $\mathcal{U}_{\mathrm{alg}}^{\infty}$. In view of Prop. 4.3 (see also Thm. A.1), $\mathcal{V}$ is necessarily a subset of $\mathcal{F}$. Thus, when investigating properties of the energy density, it is actually enough to consider elements in $\mathcal{F}_{\omega}$ for arbitrary $\omega \in \mathcal{S}_{\mathrm{qH}}$.

Accordingly, let $\omega \in \mathcal{S}_{\mathrm{qH}}$ be arbitrarily chosen. Then we define the one-point function as a linear map from $\mathcal{F}_{\omega}$ to $\mathscr{D}^{\prime}(M)$ by

$$
\Phi[\ell](f)=\left\langle\psi, \Phi_{\omega}(f) \varphi\right\rangle,
$$


for $\ell \in \mathcal{F}_{\omega}$ as in (4.5), and this is necessarily a weak solution to the Klein-Gordon equation. Similarly, the two-point function is a weak bisolution defined by

$$
\Phi^{\otimes 2}[\ell](f, g)=\left\langle\Phi_{\omega}(\bar{f}) \psi, \Phi_{\omega}(g) \varphi\right\rangle,
$$

which satisfies the commutator property

$$
\Phi^{\otimes 2}[\ell](f, g)-\Phi^{\otimes 2}[\ell](g, f)=i E(f, g) \ell(\mathbf{1})
$$

as may be seen by a short argument using the Weyl relations and Leibniz' rule.

The microlocal properties of the one- and two-point functions are easily determined using the calculus of Prop. 3.2. Starting with the observation that

$$
\langle\Phi(\bar{f}) \psi, \varphi\rangle=\Phi[\ell](f)=\langle\psi, \Phi(f) \varphi\rangle,
$$

for $\psi, \varphi \in \operatorname{Had}(\omega)$, the Hadamard condition (4.4) and Prop. 3.2(ii) imply

$$
\left(\mathcal{N}^{-}\right)^{\dagger} \supset \mathrm{WF}(\Phi(-) \psi) \supset \mathrm{WF}(\Phi[\ell]) \subset \mathrm{WF}(\Phi(\cdot) \varphi) \subset \mathcal{N}^{-}
$$

so the one-point function obeys $\operatorname{WF}(\Phi[\ell]) \subset \mathcal{N}^{+} \cap \mathcal{N}^{-}=\emptyset$ and is therefore smooth. Turning to the two-point function, Eq. (4.6), Prop. 3.2(iii) and the Hadamard condition (4.4) give

$$
\begin{aligned}
\mathrm{WF}\left(\Phi^{\otimes 2}[\ell]\right) & \subset\left(\mathrm{WF}(\Phi(\cdot) \psi)^{\dagger} \cup Z\right) \times(\mathrm{WF}(\Phi(\cdot) \varphi) \cup Z) \\
& \subset\left(\mathcal{N}^{+} \cup Z\right) \times\left(\mathcal{N}^{-} \cup Z\right) .
\end{aligned}
$$

In the special case in which $\ell$ is a state, the above inclusion and the commutator property combine to yield the stronger result that $\mathrm{WF}\left(\Phi^{\otimes 2}[\ell]\right)$ is contained in the righthand side of Eq. (4.3) and that the two-point function therefore takes the Hadamard form. By polarisation, it follows that the normal ordered two-point function

$$
: \Phi^{\otimes 2}:[\ell]=\Phi^{\otimes 2}[\ell]-\ell(\mathbf{1}) \Phi^{\otimes 2}\left[\omega_{0}\right]
$$

(relative to the reference state $\omega_{0}$ fixed in Sec.4.1) can be identified with a smooth function on $M \times M$ for each $\ell \in \mathcal{F}$. The point-split normal ordered energy density is defined in terms of this quantity by

$$
: T:[\ell]\left(x, x^{\prime}\right)=\frac{1}{2}\left(m^{2}+\sum_{\mu=0}^{s} e_{\mu}^{a} \nabla_{a} \otimes e_{\mu}^{a^{\prime}} \nabla_{a^{\prime}}\right): \Phi^{\otimes 2}:[\ell]\left(x, x^{\prime}\right)
$$

and is also smooth on $M \times M$; finally, the normal ordered energy density itself is given (cf. (4.1) ) by

$$
\boldsymbol{\varrho}[\ell](x)=g_{00}(x)^{1 / 2}: T:[\ell](x, x) .
$$

All the quantities defined so far clearly extend to finite linear combinations of functionals $\ell$ in $\mathcal{F}_{\omega}$ as $\omega$ ranges over $\mathcal{S}_{\mathrm{qH}}$, and hence to $\ell \in \mathcal{F}$. In particular, $\varrho$ is defined on $\mathcal{S}$. As will be proved in Sec. A.2 the spatial integral of this quantity generates the dynamics. 
Proposition 4.4 For all $t \in \mathbb{R}$ we have

$$
\int_{\Sigma} d \mu(\underline{x}) \ell([\varrho(t, \underline{x}), A])=\left.\frac{1}{i} \frac{d}{d s} \ell\left(\alpha_{s} A\right)\right|_{s=0}, \quad A \in \mathcal{U}_{\mathrm{alg}}^{\infty}, \ell \in \mathcal{V},
$$

where $\mathcal{V}$ is, as in Sec. 圆, the vector space generated by $\mathcal{S}$ and $\mathcal{U}_{\text {alg }}^{\infty}$.

Eq. (4.8) clearly implies that both the assumptions (i) and (ii) made on the dynamics in Sect. 2 are satisfied: to derive (ii) one simply observes that the right-hand side is $t$ independent, while (i) follows on replacing $A$ by $\alpha_{t} A$.

\subsection{The quantum weak energy inequality}

The last step in justifying the structural assumptions of Sec. 2 for our model is the identification of a state $\omega$ obeying a suitable QWEI. For this purpose, $\omega$ may be chosen to be any state in $\mathcal{S}$ whose 2-point function $\Phi^{\otimes 2}[\omega](x, y)$ is invariant under $x \mapsto \tau_{t} x, y \mapsto \tau_{t} x$ for any $t \in \mathbb{R}^{8}$

Proposition 4.5 Let the (unrenormalised) point-split energy density $T_{0} \in \mathscr{D}^{\prime}(M \times M)$ be defined by

$$
T_{0}=\frac{1}{2}\left(m^{2}+\sum_{\mu=0}^{s} e_{\mu}^{a} \nabla_{a} \otimes e_{\mu}^{a^{\prime}} \nabla_{a^{\prime}}\right) \Phi^{\otimes 2}[\omega]
$$

and define $\Gamma_{\underline{x}}: \mathbb{R} \rightarrow M \times M$ by $\Gamma_{\underline{x}}(t)=(t, \underline{x} ; 0, \underline{x})$. Then:

i) the pull-back $\Gamma_{\underline{x}}^{*} T_{0}$ exists as an element of $\mathscr{D}^{\prime}(\mathbb{R})$ with

$$
\mathrm{WF}\left(\Gamma_{\underline{x}}^{*} T_{0}\right) \subset\{(t, \zeta) \mid \zeta>0\} ;
$$

ii) $\Gamma_{\underline{x}}^{*} T_{0}$ is positive-type in the sense that

$$
\Gamma_{\underline{x}}^{*} T_{0}(\bar{f} \star \widetilde{f}) \geq 0 \quad \text { for all } f \in \mathscr{D}(\mathbb{R}),
$$

where $\widetilde{f}(t)=f(-t)$. Furthermore, $\Gamma_{\underline{x}}^{*} T_{0}$ is a tempered distribution whose Fourier transform is a positive measure with respect to which $(-\infty, u]$ has finite measure, polynomially bounded in $u$.

Proof: i) is a direct calculation, using the fact that $\mathrm{WF}\left(T_{0}\right)$ is contained in $\mathrm{WF}\left(\Phi^{\otimes 2}[\omega]\right)$ which (since all covectors contained therein are null) has trivial intersection with the conormal bundle

$$
N_{\Gamma_{\underline{x}}}=\left\{\left((t, \underline{x} ; 0, \underline{x}),\left(0, \underline{\xi} ; \zeta^{\prime}, \underline{\xi}^{\prime}\right)\right): \zeta^{\prime} \in \mathbb{R}, \underline{\xi}, \underline{\xi}^{\prime} \in T_{\underline{x}}^{*} \Sigma\right\}
$$

of $\Gamma_{\underline{x}}$. Part ii) follows because $\Gamma_{\underline{x}}^{*} T_{0}(f \star \widetilde{g})=\gamma_{\underline{x}}^{(2) *} T_{0}(f \otimes g)$ where $\gamma_{\underline{x}}^{(2)}: \mathbb{R}^{2} \rightarrow M \times M$ is defined by $\gamma_{\underline{x}}^{(2)}\left(t, t^{\prime}\right)=\left(t, \underline{x} ; t^{\prime}, \underline{x}\right.$ ). (This map has conormal bundle $N_{\gamma_{\underline{x}}(2)}=N_{\gamma_{\underline{x}}} \times N_{\gamma_{\underline{x}}}$, where

$$
N_{\gamma_{\underline{x}}}=\left\{(t, \underline{x} ; 0, \underline{\xi}): t \in \mathbb{R}, \underline{\xi} \in T_{\underline{x}}^{*} \Sigma\right\}
$$

\footnotetext{
${ }^{8}$ Such states certainly exist: for example, one could use a ground- or KMS-state, but only the invariance and Hadamard properties are needed below.
} 
is the conormal bundle for $\gamma_{\underline{x}}: t \mapsto(t, \underline{x})$. But $N_{\gamma_{\underline{x}}}$ contains no null covectors, so the pullback $\gamma_{\underline{x}}^{(2) *} T_{0}$ is well-defined.) Since $T_{0}$ is positive type in the sense that $T_{0}(\bar{F} \otimes F) \geq 0$ for $F \in \mathscr{D}(M)$, it follows by Theorem 2.2 in [12] that $\gamma_{\underline{x}}^{(2) *} T_{0}$ is also positive type in this sense and that (4.9) holds. The remaining statements follow from Theorem A.11 in Sec. A.3, a variant of the Bochner-Schwartz theorem.

With the above definitions, the arguments of Sec. 5 of [12] may be adapted straightforwardly ${ }^{9}$ to show that $\omega$ obeys a static QWEI in the sense described in Sec. 2 with respect to the set of states $\mathcal{S}$, where

$$
q(g, \underline{x})=\int_{\mathbb{R}} d u|\widehat{g}(u)|^{2} Q(u, \underline{x})
$$

and

$$
Q(u, \underline{x})=\frac{1}{2 \pi^{2}} \int_{(-\infty, u)} d \zeta\left[\Gamma_{\underline{x}}^{*} T_{0}\right]^{\wedge}(\zeta) .
$$

In fact, because $\widehat{g}$ is smooth, the static QWEI would be unchanged if we had instead used the integration range $(-\infty, u]$ to define $Q$; however, the above definition is technically more convenient, as it entails that $Q(u, \underline{x})$ is left-continuous in $u$ for each fixed $\underline{x}$. We also note that $Q$ is a well-defined nonnegative measureable function on $\mathbb{R} \times \Sigma$ as a consequence of Prop. 4.5(ii); a further consequence of which is that $Q(u, \underline{x})$ is polynomially bounded in $u$ for each fixed $\underline{x} \in \Sigma$.

The final property required of $Q$ is proved in Sec. A.4.

Proposition 4.6 For each $u \in \mathbb{R}, Q(u, \cdot) \in L^{1}(\Sigma, d \mu)$; furthermore, the function $\mathfrak{Q}(u):=\int_{\Sigma} d \mu(\underline{x}) Q(u, \underline{x})$ is monotonically increasing, left-continuous and polynomially bounded in $u$.

This then implies that $q(g ;.) \in L^{1}(\Sigma, d \mu)$, thus $\omega$ fulfills a static QWEI, but we even have

Theorem 4.7 The state $\omega$ fulfills a limiting static QWEI (with respect to $\mathcal{S}$ ), and all states in $\mathcal{S}$ fulfill a static $Q W E I$.

Proof: Note that

$$
\begin{aligned}
\frac{1}{\left\|g_{\lambda}^{2}\right\|_{L^{1}}} \int_{\Sigma} d \mu(\underline{x}) q\left(g_{\lambda} ; \underline{x}\right) & =\frac{1}{\left\|g^{2}\right\|_{L^{1}}} \int_{\Sigma} d \mu(\underline{x}) \int_{\mathbb{R}} d u|\widehat{g}(u)|^{2} Q(\lambda u, \underline{x}) \\
& =\frac{1}{\left\|g^{2}\right\|_{L^{1}}} \int_{\mathbb{R}} d u|\widehat{g}(u)|^{2} \mathfrak{Q}(\lambda u),
\end{aligned}
$$

where Fubini's theorem has been used. Since $\mathfrak{Q}$ is polynomially bounded and $\widehat{g}$ is of rapid decrease, for any $\epsilon>0$ there exists $U>0$ such that

$$
\int_{|u|>U} d u|\widehat{g}(u)|^{2} \mathfrak{Q}(\lambda u)<\epsilon\left\|g^{2}\right\|_{L^{1}}, \quad \lambda \in(0,1) .
$$

\footnotetext{
${ }^{9}$ There are two main differences: first, a change of parametrisation in the worldline; second, in [12], the state $\omega$ [there denoted $\omega_{0}$ ] was additionally assumed to be a ground state of the time evolution, which has the effect of limiting the $\zeta$ integration in (4.10) to $[0, u)$, but is not otherwise needed in the derivation.
} 
Thus

$$
\begin{aligned}
\frac{1}{\left\|g^{2}\right\|_{L^{1}}} \int_{\mathbb{R}} d u|\widehat{g}(u)|^{2} \mathfrak{Q}(\lambda u) & \leq \frac{1}{\left\|g^{2}\right\|_{L^{1}}} \int_{|u|<U} d u|\widehat{g}(u)|^{2} \mathfrak{Q}(\lambda u)+\epsilon \\
& \leq 2 \pi \sup _{|u|<U} \mathfrak{Q}(\lambda u)+\epsilon \\
& =2 \pi \mathfrak{Q}(\lambda U)+\epsilon
\end{aligned}
$$

for all $\lambda \in(0,1)$, using monotonicity and left-continuity of $\mathfrak{Q}$. Putting this together with Eq. (4.11) and taking the limit $\lambda \rightarrow 0^{+}$, we have

$$
\limsup _{\lambda \rightarrow 0^{+}} \frac{1}{\left\|g_{\lambda}^{2}\right\|_{L^{1}}} \int_{\Sigma} d \mu(\underline{x}) q\left(g_{\lambda} ; \underline{x}\right) \leq \limsup _{u \rightarrow 0^{+}} \mathfrak{Q}(u)+\epsilon=\lim _{u \rightarrow 0^{+}} \mathfrak{Q}(u)+\epsilon,
$$

using monotonicity of $\mathfrak{Q}(u)$ again. Since $\epsilon$ was arbitrary, the left-hand side is bounded uniformly in $g$ by $\mathfrak{Q}(0+):=\lim _{u \rightarrow 0^{+}} \mathfrak{Q}(u)$. Thus $\omega$ fulfills a limiting static QWEI with $0 \leq \Gamma_{\Sigma} \leq \mathfrak{Q}(0+)$; the remaining statement follows as indicated in Remark (i) following Def. [2.1],

Finally, we strengthen the link between quiescence and ground states as follows:

Theorem 4.8 If $\omega \in \mathcal{S}$ is a non-degenerate ground state with a mass gap and vanishing one-point function then $\omega$ is quiescent.

Proof: In the GNS representation $\left(\mathcal{H}_{\omega}, \pi_{\omega}, \Omega_{\omega}\right)$ induced by $\omega$, the dynamics is generated by a self-adjoint operator $H$ with spectrum $\sigma(H) \subset\{0\} \cup\left[m_{0}, \infty\right)$ for some $m_{0}>0$ and such that 0 is a simple eigenvalue with eigenvector $\Omega$. Thus for any $F, G \in \mathscr{D}(M)$,

$$
\begin{aligned}
\Phi^{\otimes 2}[\omega]\left(F, \tau_{t *} G\right) & =\left\langle\Phi_{\omega}(\bar{F}) \Omega_{\omega}, e^{i H t} \Phi_{\omega}(G) \Omega_{\omega}\right\rangle \\
& =\int e^{i \zeta t} d\left\langle\Phi_{\omega}(\bar{F}) \Omega_{\omega}, E_{\zeta} \Phi_{\omega}(G) \Omega_{\omega}\right\rangle,
\end{aligned}
$$

where $d E_{\zeta}$ is the spectral measure for $H$. Due to the spectral properties of $H$, the nondegeneracy of $\omega$ and the vanishing one-point functions $\left\langle\Omega_{\omega}, \Phi_{\omega}(G) \Omega_{\omega}\right\rangle$, the (finite) measure $d\left\langle\Phi_{\omega}(\bar{F}) \Omega_{\omega}, E_{\zeta} \Phi_{\omega}(G) \Omega_{\omega}\right\rangle$ is in fact supported in $\left[m_{0}, \infty\right)$. Similarly, since time translation commutes with $\nabla_{a}$,

$$
T_{0}\left(F, \tau_{t *} G\right)=\int e^{i \zeta t} d \rho_{F, G}(\zeta)
$$

where $d \rho_{F, G}$ is finite and supported in $\left[m_{0}, \infty\right)$.

Now take any $f, g \in \mathscr{D}(\mathbb{R})$ and a sequence $\chi_{n} \rightarrow \delta_{\underline{x}}$ in $\mathscr{D}_{T^{* \Sigma}}^{\prime}(\Sigma)$. Put $F_{n}=f \otimes \chi_{n}$, $G_{n}=g \otimes \chi_{n}$. Then for any $h \in \mathscr{D}(\mathbb{R})$, we have

$$
\begin{aligned}
\int \widehat{h}(\zeta) d \rho_{F_{n}, G_{n}}(\zeta) & =\int d t h(t) T_{0}\left(F_{n}, \tau_{t *} G_{n}\right) \\
& =T_{0}\left(F_{n}, H_{n}\right) \\
& \rightarrow \gamma_{\underline{x}}^{(2) *} T_{0}(f, h \star g) \\
& =\Gamma_{\underline{x}}^{*} T_{0}(f \star \widehat{h \star g}) \\
& =(2 \pi)^{-1} \widehat{\Gamma_{\underline{*}}^{*} T_{0}}(\widetilde{\widehat{f}} \widehat{g} \widehat{h}),
\end{aligned}
$$


where $H_{n}=[h \star g] \otimes \chi_{n}$. Furthermore, a similar argument shows that

$$
\int d \rho_{F_{n}, G_{n}}(\zeta) \rightarrow(2 \pi)^{-1} \widehat{\Gamma_{\underline{x}}^{*} T_{0}}(\widetilde{\widehat{f}} \widehat{g})
$$

which, considering the case $f=\bar{g}$ first and then polarising, implies

$$
\left|\int \widehat{h}(\zeta) d \rho_{F_{n}, G_{n}}(\zeta)\right| \leq C_{f, g} \sup |\widehat{h}|
$$

This provides the necessary uniformity to conclude that

$$
\int \widehat{h}(\zeta) d \rho_{F_{n}, G_{n}}(\zeta) \rightarrow \widehat{\Gamma_{\underline{x}}^{*} T_{0}}(\widetilde{\widetilde{f}} \widehat{g} \hat{h})
$$

for all $h \in \mathscr{S}(\mathbb{R})$. Choosing $h$ so that $\operatorname{supp} \widehat{h} \cap\left[m_{0}, \infty\right)=\emptyset$, and using the fact that

linear combinations of functions of the form $\widetilde{\widehat{f}} \widehat{g}$ are dense in $\mathscr{S}(\mathbb{R})$, we deduce that $\widehat{\Gamma_{\underline{x}}^{*} T_{0}}$ is supported in $\left[m_{0}, \infty\right)$.

It follows from this that $Q(u, \underline{x})$ (for each $\underline{x}$ ) and $\mathfrak{Q}(u)$ are supported in $\left[m_{0}, \infty\right)$. Since we already know that $\omega$ fulfills a limiting static QWEI with $0 \leq \Gamma_{\Sigma} \leq \mathfrak{Q}(0+)$ we may now conclude that $\Gamma_{\Sigma}=0$ and that $\omega$ is therefore quiescent.

\section{Conclusion}

We have now seen that a $C^{*}$-dynamical system $\left(\mathcal{A},\left\{\alpha_{t}\right\}_{t \in \mathbb{R}}\right)$, together with a suitable class of states and an energy-density can be constructed for the free scalar field on a static, spatially compact globally hyperbolic spacetime such that the assumptions relevant to Sec. 2 are fulfilled. Consequently, we obtain from Thm. 2.3 that passive states $\omega^{p}$ exist for the dynamical system $\left(\mathcal{A},\left\{\alpha_{t}\right\}_{t \in \mathbb{R}}\right)$. It may be worth mentioning again that $\mathcal{A}$ does not coincide with the Weyl-algebra $\mathfrak{A}[S, \sigma]$ for the free scalar field on the given static spacetime, so it is not a priori clear if $\omega^{p}$ induces a passive state (in a $W^{*}$-sense) on $\mathfrak{A}[S, \sigma]$. This would follow if $\omega^{p}$ were found to be normal to any quasifree Hadamard state, and this is in fact expected to hold in view of the results of [37. Furthermore, as shown by Thm. 2.6 and Cor. 2.7, $\omega^{p}$ is normal when energy-compactness holds which is believed to be generically fulfilled in quantum field theoretical models relevant to particle physics.

It should be noted that this technical complication does not arise in the case of the free Dirac field, and we should like to emphasize that our methods apply also in this case, so that also for the case of the Dirac field on a static, spatially compact and globally hyperbolic spacetime one would be led to the conclusion that there is a $C^{*}$-dynamical system together with a class of states and an energy density fulfilling the assumptions made in Sec. 2 .

In the course of this work, we have also proved various new results concerning the free scalar field. In particular, we have demonstrated that the static QWEI bounds obtained in [12] exhibit sufficient spatial regularity to be integrable. We hope also to have demonstrated the utility of the reformulation (introduced in [40] and developed further 
here) of the Hadamard condition as a wave-front set condition on Hilbert space-valued distributions. We expect both this and the microlocal calculus of Banach space-valued distributions given in Prop. 3.2 to have further applications.

The results of Sec. 4 combine with those of Sec. 2 to give substance to the connection between the three conditions of dynamical stability mentioned towards the end of the Introduction. This connection corroborates the point of view originally advocated by Ford [17] that there should be local constraints on the amount and duration of negative energy densities in physical quantum states in order that no violations of the second law of thermodynamics can build up at macroscopic scales. However, we re-emphasise that our results indicate a still deeper equivalence between natural conditions of dynamical stability at microscopic, mesoscopic and macroscopic scales.

Of course the question arises how far such an equivalence may be extended from the situation studied in the present article so as to apply to interacting quantum fields in generic curved spacetimes without time-symmetries, and hence be regarded as universal. The main difficulty seems to lie in a missing counterpart of the concept of passivity when the dynamics of a system is no longer described by a group of automorphisms with time-independent generators. A further study of possible generalizations of the passivity concept applicable to quantum field theory on generic curved spacetimes and their interconnections to microlocal spectrum condition and QWEIs appears, in the light of the present results, to offer an interesting line of investigation.

\section{A Technical appendix}

\section{A.1 Stability of $\mathcal{S}$}

Theorem A.1 Let $\omega$ be a quasifree Hadamard state. Then $\operatorname{Had}(\omega)$ is invariant under the action of any element in $\beta_{\omega \omega_{0}}(\mathcal{B})$ where $\mathcal{B}$ is the *-algebra finitely generated by Weyl operators, elements of $\mathcal{A}^{\infty}$ and elements of $\mathcal{U}^{\infty}$. Furthermore, if $A \in \beta_{\omega \omega_{0}}(\mathcal{B})$ then $\left[\Phi_{\omega}(\cdot), A\right] \in \mathscr{D}^{\prime}\left(M, \mathcal{L}\left(\mathcal{H}_{\omega}\right)\right)$ with empty wave-front set. In particular, $A \Omega_{\omega} \in \operatorname{Had}(\omega)$ for any $A \in \beta_{\omega \omega_{0}}(\mathcal{B})$.

Proof: We will use the following conventions. We will write $W(f)$ for the Weyl operators $\pi_{\omega}(\mathrm{W}(E f))$ in the GNS-representation of $\omega$, and $\Phi(f)$ for the corresponding generators $\Phi_{\omega}(f)\left(\mathrm{e}^{i \Phi(f)}=W(f)\right)$. Moreover, we identify elements $A \in \mathcal{M}_{\omega_{0}}\left(\right.$ via $\left.\beta_{\omega \omega_{0}}\right)$ with elements in $\mathcal{M}_{\omega}$, i.e., we write $A$ in place of $\beta_{\omega \omega_{0}}(A)$.

Observing these conventions, it is sufficient to prove that $\operatorname{Had}(\omega)$ is invariant under Weyl operators, elements of $\mathcal{A}^{\infty}$ or operators of the form $e^{i A}$ for $A=A^{*} \in \mathcal{A}^{\infty}$ and that the required wave-front set condition holds for the commutators of $\Phi$ with such operators. This will be accomplished in a series of lemmas, starting with the case of Weyl operators.

Lemma A.2 For each $g \in \mathscr{D}(M)$ and $h \in \mathscr{D}(M ; \mathbb{R})$, we have $W(h) D(\Phi(g)) \subset D(\Phi(g))$ and

$$
[\Phi(g), W(h)] \psi=-E(g, h) W(h) \psi, \quad \psi \in D(\Phi(g))
$$

and $[\Phi(\cdot), W(h)] \in \mathscr{D}^{\prime}\left(M, \mathcal{L}\left(\mathcal{H}_{\omega}\right)\right)$ with empty wave-front set. 
Proof: The domain property and (A.1) are standard. Since Eq. (A.1) implies $\|[\Phi(g), W(h)]\|=|E(g, h)|$, the remaining statements follow immediately from Prop. $3.2(\mathrm{i})$ and the fact that $\mathrm{WF}(E(\cdot, h))=\emptyset$.

Lemma A.3 For each $g \in \mathscr{D}(M)$ and $A \in \mathcal{A}^{\infty}$, we have $A D(\Phi(g)) \subset D(\Phi(g))$ and

$$
[\Phi(g), A] \psi=B(g) \psi
$$

where $B(g) \in \mathcal{A}^{\infty}$ is given by

$$
B(g)=\int d t f(t) E\left(\tau_{t *} h, g\right) W\left(\tau_{t *} h\right) .
$$

Furthermore, $B(\cdot) \in \mathscr{D}^{\prime}\left(M, \mathcal{L}\left(\mathcal{H}_{\omega}\right)\right)$ and $\mathrm{WF}(B(\cdot))=\emptyset$.

Proof: Suppose that $g$ is real-valued and that $A$ is of the form $\alpha_{f} W(h)$ [the extension to finite linear combinations and products of such operators is immediate]. To establish the domain property, it suffices to show that $s \mapsto s^{-1}\|(W(s g)-\mathbf{1}) A \psi\|$ is bounded for $\psi \in D(\Phi(g))$. Now if $X$ is any bounded operator one may check straightforwardly that

$$
\|X A \psi\| \leq \int d t|f(t)|\left\|X \alpha_{t}(W(h)) \psi\right\| .
$$

Since $(W(s g)-\mathbf{1}) W(h)=W(h)\left[e^{-i s E(g, h)} W(s g)-\mathbf{1}\right]$, we therefore have

$$
\begin{aligned}
s^{-1}\|(W(s g)-\mathbf{1}) A \psi\| & \leq s^{-1} \int d t|f(t)|\left\|\left(e^{-i s E\left(g, \tau_{t *} h\right)} W(s g)-\mathbf{1}\right) \psi\right\| \\
& \leq s^{-1} \int d t|f(t)|\left\{\|(W(s g)-\mathbf{1}) \psi\|+\left|e^{-i s E\left(g, \tau_{t *} h\right)}-1\right|\|\psi\|\right\} \\
& \leq\|f\|_{1}\left\{\|\Phi(g) \psi\|+\sup _{t \in \operatorname{supp} f}\left|E\left(g, \tau_{t *} h\right)\right|\|\psi\|\right\} .
\end{aligned}
$$

(Note that the supremum exists as $t \mapsto E\left(g, \tau_{t *} h\right)$ is continuous.) Thus we have shown that $A \psi \in D(\Phi(g))$ for any real-valued $g$ : since in general $D(\Phi(g))=D(\Phi(\operatorname{Re} g)) \cap D(\Phi(\operatorname{Im} g))$, the result extends immediately to general $g \in \mathscr{D}(M)$.

Eqs. (A.2) and (A.3) are now easily checked using the identity

$$
W(f)^{*} \Phi(g) W(f)=\Phi(g)+E(f, g) \mathbf{1}
$$

which holds on $D(\Phi(g))(g \in \mathscr{D}(M))$.

Next, define $K(g) \in C_{0}^{\infty}(\mathbb{R})$ by

$$
K(g)(t)=f(t) E\left(\tau_{t *} h, g\right) .
$$

Lemma A.4 $K(\cdot) \in \mathscr{D}^{\prime}\left(M, L^{1}(\mathbb{R})\right)$ with $\mathrm{WF}(K)=\emptyset$.

Proof: Note first that

$$
\|K(g)\|_{L^{1}} \leq\|f\|_{L^{1}} \sup _{t \in \operatorname{supp} f}\left|E\left(\tau_{t *} h, g\right)\right| \leq C \sup _{x}|g(x)|
$$

so $K(\cdot) \in \mathscr{D}^{\prime}\left(M, L^{1}(\mathbb{R})\right)$. For each $(x, k) \in \dot{T}^{*} M$, choose $\mathcal{O}$ and $\phi$ obeying condition (A) of Sec. 3 and define coordinates on $\mathcal{O}$ via $y^{\mu}=\left\langle\zeta^{\mu}, \phi(y)\right\rangle$ where $\zeta^{\mu}$ is an arbitrary fixed basis of $T_{x}^{*} M$. Then for each $\chi \in C_{0}^{\infty}(\mathcal{O}), E\left(\tau_{t *} h, \chi e^{i \lambda\langle\eta, \phi\rangle}\right)$ is the usual Fourier transform ${ }^{10}$ at

\footnotetext{
${ }^{10}$ See footnote 6
} 
$\lambda \ell_{\mu}$ (where $\left.T_{x}^{*} M \ni \ell=\ell_{\mu} \zeta^{\mu}\right)$ of $F_{t}=-|\operatorname{det} \boldsymbol{g}|^{1 / 2}\left(\chi E \tau_{t *} h\right) \circ \kappa^{-1}$ where $\kappa: y \mapsto y^{\mu}$ is the coordinate map. Since $F_{t} \in C_{0}^{\infty}(\kappa(\mathcal{O}))$ with derivatives varying continuously in $t$, and since supp $f$ is compact, for any relatively compact neigbourhood $V$ of $k$ in $T_{p}^{*} M$ there exist constants $C_{N}$ such that

$$
\sup _{\ell \in V}\left|E\left(\tau_{t *} h, \chi e^{i \lambda\langle\ell, \phi\rangle}\right)\right| \leq \frac{C_{N}}{1+\lambda^{N}}, \quad \lambda \in \mathbb{R}^{+}, t \in \operatorname{supp} f,
$$

for each $N=1,2, \ldots$ Accordingly, each $(x, k) \in \dot{T}^{*} M$ is a regular directed point for $K$, so $\mathrm{WF}(K)=\emptyset$.

It now follows by Prop. 3.2(i) and the bound $\|B(g)\| \leq\|K(g)\|_{L^{1}}$ obtained from Eq. (A.3) that $B(\cdot) \in \mathscr{D}^{\prime}\left(M, \mathcal{L}\left(\mathcal{H}_{\omega}\right)\right)$ and $\mathrm{WF}(B(\cdot))=\emptyset$.

Lemma A.5 For each $g \in \mathscr{D}(M)$ and $A \in \mathcal{A}^{\infty}$, we have $e^{i A} D(\Phi(g)) \subset D(\Phi(g))$ and

$$
\left[\Phi(g), e^{i A}\right] \psi=C(g) \psi, \quad \psi \in D(\Phi(g)),
$$

where $C(g)$ is defined by the norm convergent series

$$
C(g)=\sum_{k=1}^{\infty} \frac{i^{k}}{k !} \sum_{j=0}^{k-1} A^{j} B(g) A^{k-j-1}
$$

we have $C(\cdot) \in \mathscr{D}^{\prime}\left(M ; \mathcal{L}\left(\mathcal{H}_{\omega}\right)\right)$ and $\mathrm{WF}(C(\cdot))=\emptyset$.

Proof: By the previous lemma, we may deduce

$$
\Phi(g) A^{k} \psi=A^{k} \Phi(g) \psi+\sum_{j=0}^{k-1} A^{j} B(g) A^{k-j-1}
$$

for $A \in \mathcal{A}^{\infty}, k \geq 1$ and $\psi \in D(\Phi(g))$. Norm convergence in (A.11) follows from the observation

$$
\sum_{k=1}^{\infty} \frac{1}{k !} \sum_{j=0}^{k-1}\|A\|^{k-1}\|B\|=e^{\|A\|}\|B(g)\|<\infty .
$$

Moreover, the same argument shows that $\|C(g) \psi\| \leq e^{\|A\|}\|B(g)\|\|\psi\|$. By Prop. [3.2(i), we now conclude that $C \in \mathscr{D}^{\prime}\left(M ; \mathcal{L}\left(\mathcal{H}_{\omega}\right)\right)$ with $\mathrm{WF}(C(\cdot))=\emptyset$ since $B$ also enjoys these properties.

The proof of Theorem A.1 is complete.

Proof of Proposition 4.3. The property $\mathcal{S}_{\mathrm{qH}} \subset \mathcal{S}$ is obvious since for each $\omega \in \mathcal{S}_{\mathrm{qH}}$ the GNS-vector $\Omega_{\omega}$ is contained in $\operatorname{Had}(\omega)$. Also, $\mathcal{S}$ is closed under finite convex combinations by its very definition. Now $\mathcal{U}_{\text {alg }}^{\infty}$ is a sub-*-algebra of $\mathcal{B}$, and therefore its action on $\operatorname{Had}(\omega)$ preserves $\operatorname{Had}(\omega)$. As $\omega \in \mathcal{S}_{\mathrm{qH}}$ was arbitrary, this implies immediately that $\mathcal{S}$ is preserved under operations in $\mathcal{U}_{\text {alg }}^{\infty}$. 


\section{A.2 The integrated energy density as the dynamical generator}

Let $\gamma_{t}: \Sigma \rightarrow M \times M$ be defined by $\gamma_{t}(\underline{x})=(t, \underline{x} ; t, \underline{x})$.

Proposition A.6 Let $\omega \in \mathcal{S}_{\mathrm{qH}}$ and let $\ell \in \mathcal{F}_{\omega}$. Then for all $t_{0} \in \mathbb{R}$ we have

$$
\left(\gamma_{t_{0}}^{*} \ell([: T:, A])\right)\left(g_{00}^{1 / 2}\right)=\left.\frac{1}{i} \frac{d}{d s} \ell\left(\alpha_{s} A\right)\right|_{s=0}
$$

for any $A \in \beta_{\omega \omega_{0}}(\mathcal{B})$.

Given this, Prop. 4.4 follows easily.

Proof of Proposition 4.4: Noting that $\mathcal{U}_{\mathrm{alg}}^{\infty} \subset \mathcal{B}$ and that any $\ell \in \mathcal{V}$ is a finite convex combination of functionals $\ell_{i} \in \mathcal{F}_{\omega_{i}}, \omega_{i} \in \mathcal{S}_{\mathrm{qH}}$, Eq. A.14 certainly holds for all $A \in \mathcal{U}_{\text {alg }}^{\infty}$ and $\ell \in \mathcal{V}$. To complete the proof, we observe that since $\ell([: T: A])$ is smooth we have

$$
\left(\gamma_{t_{0}}^{*} \ell([: T: A])\right)\left(g_{00}^{1 / 2}\right)=\int d \mu(\underline{x}) \ell\left(\left[\varrho\left(t_{0}, \underline{x}\right), A\right]\right)
$$

and the result follows.

Proof of Prop. A.6: In the following subsections, we first establish Eq. (A.14) for Weyl

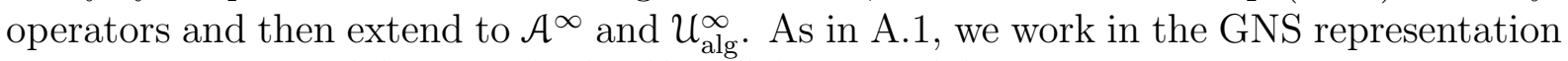
of $\omega$, and write $W(f)$ for $\pi_{\omega}(\mathrm{W}(E f)), \Phi(f)$ for $\Phi_{\omega}(f)$, and we identify $A \in \mathcal{M}_{\omega_{0}}$ with $\beta_{\omega \omega_{0}}(A) \in \mathcal{M}_{\omega}$ without writing the $\beta_{\omega \omega_{0}}$.

\section{A.2.1 Weyl operators}

Let us first observe that, for any $A \in \mathcal{B}$, we have

$$
\ell\left(\left[: \Phi^{\otimes 2}:, A\right]\right)(f, g)=\langle\Phi(\bar{f}) \psi,[\Phi(g), A] \varphi\rangle+\left\langle\left[\Phi(\bar{f}), A^{*}\right] \psi, \Phi(g) \varphi\right\rangle,
$$

as may be seen by a straightforward calculation. In the particular case $A=W(h)$ for $h \in \mathscr{D}(M ; \mathbb{R})$, this gives

$$
\ell\left(\left[: \Phi^{\otimes 2}:, W(h)\right]\right)(x, y)=-\Phi\left[\ell_{W(h)}\right](x) E h(y)-E h(x) \Phi\left[{ }_{W(h)} \ell\right](y),
$$

and, noting that each term in A.15 is a product of smooth Klein-Gordon solutions, we use the following lemma.

Lemma A.7 Suppose $u$ and $v$ are $C^{\infty}$ solutions to the Klein-Gordon equation on $(M, \boldsymbol{g})$ and define

$$
\rho(x)=\frac{1}{2} g_{00}(x)^{1 / 2}\left(\left.\left.\sum_{\mu=0}^{s}\left(e_{\mu}^{a} \nabla_{a} u\right)\right|_{x}\left(e_{\mu}^{a} \nabla_{a} v\right)\right|_{x}+m^{2} u(x) v(x)\right) .
$$

Then for any $t_{0} \in \mathbb{R}$,

$$
\int_{\Sigma} d \mu(\underline{x}) \rho\left(t_{0}, \underline{x}\right)=\frac{1}{2} \sigma\left(\xi^{a} \nabla_{a} u, v\right),
$$

and in the particular case $u=E h$,

$$
\int_{\Sigma} d \mu(\underline{x}) \rho\left(t_{0}, \underline{x}\right)=\frac{1}{2} \int_{M} d \operatorname{vol}_{\boldsymbol{g}}(x) v(x)\left(\xi^{a} \nabla_{a} h\right)(x) .
$$


Proof: We have

$$
\begin{aligned}
\sigma\left(\xi^{a} \nabla_{a} u, v\right) & =\int_{\Sigma} d \mu(\underline{x}) g_{00}^{1 / 2}\left[\left(e_{0}^{a} \nabla_{a} u\right)\left(e_{0}^{b} \nabla_{b} v\right)-v\left(e_{0}^{a} \nabla_{a}\right)^{2} u\right] \\
& =\int_{\Sigma} d \mu(\underline{x}) g_{00}^{1 / 2}\left[\left(e_{0}^{a} \nabla_{a} u\right)\left(e_{0}^{b} \nabla_{b} v\right)-\delta^{i j} v \nabla_{a} e_{i}^{a} e_{j}^{b} \nabla_{b} u+m^{2} u v\right] \\
& =2 \int_{\Sigma} d \mu(\underline{x}) \rho(t, \underline{x}),
\end{aligned}
$$

where in the second step we have used the Klein-Gordon equation and in the third step, Gauss' theorem. Applying this in the particular case where $u=E h$ for $h \in \mathscr{D}(M)$ and using the fact that $\xi^{a} \nabla_{a}$ commutes with $E$, we obtain

$$
2 \int_{\Sigma} d \mu(\underline{x}) \rho(t, \underline{x})=\sigma\left(\xi^{a} \nabla_{a} E h, v\right)=\sigma\left(E \xi^{a} \nabla_{a} h, v\right)=\int_{M} d \operatorname{vol}_{\boldsymbol{g}}(x) v(x)\left(\xi^{a} \nabla_{a} h\right)(x)
$$

as required.

Using this result and (A.15) we have

$$
\begin{aligned}
\int_{\Sigma} d \mu(\underline{x}) \ell([\varrho(t, \underline{x}), W(h)]) & =-\frac{1}{2} \Phi\left[\ell_{W(h)}+{ }_{W(h)} \ell\right]\left(\xi^{a} \nabla_{a} h\right) \\
& =-\left\langle\psi, \frac{1}{2}\left\{\Phi\left(\xi^{a} \nabla_{a} h\right), W(h)\right\} \varphi\right\rangle \\
& =\left.\frac{1}{i} \frac{d}{d s} \ell\left(\alpha_{s} W(h)\right)\right|_{s=0} .
\end{aligned}
$$

Here we have used

Proposition A.8 If $\psi, \varphi \in \operatorname{Had}(\omega)$ then $s \mapsto\left\langle\psi, \alpha_{s} W(f) \varphi\right\rangle$ is continuously differentiable with derivative

$$
\frac{d}{d s}\left\langle\psi, \alpha_{s} W(f) \varphi\right\rangle=-i\left\langle\psi, \frac{1}{2}\left\{\Phi\left(\xi^{a} \nabla_{a} \tau_{s *} f\right), \alpha_{s} W(f)\right\} \varphi\right\rangle .
$$

Proof: It is enough to establish differentiability and the form of the derivative for $s=0$. We begin with two observations. First, if $\varphi \in \operatorname{Had}(\omega)$ and $f \rightarrow 0$ in $\mathscr{D}(M ; \mathbb{R})$ then $W(f) \varphi \rightarrow \varphi$ since

$$
\|W(f) \varphi-\varphi\| \leq \sup _{t \in \mathbb{R} \backslash\{0\}} t^{-1}\|(W(t f)-\mathbf{1}) \varphi\|=\|\Phi(f) \varphi\| \rightarrow 0
$$

using the fact that $\Phi(\cdot) \varphi \in \mathscr{D}^{\prime}\left(M, \mathcal{H}_{\omega}\right)$. Second,

$$
\tau_{s *} f=f-s \xi^{a} \nabla_{a} f+R(s),
$$

where $s^{-1} R(s) \rightarrow 0$ in $\mathscr{D}(M)$ as $s \rightarrow 0$.

Using the Weyl relations,

$$
\begin{aligned}
s^{-1}\left(W\left(\tau_{s *} f\right)-W(f)\right) \varphi= & s^{-1}\left(e^{i E\left(\tau_{s *} f, f\right) / 2}-1\right) W\left(\tau_{s *} f-f\right) W(f) \varphi \\
& +s^{-1}\left[W\left(\tau_{s *} f-f\right)-\mathbf{1}\right] W(f) \varphi
\end{aligned}
$$


and if $\varphi \in \operatorname{Had}(\omega)$ we may use the above observations and the invariance of $\operatorname{Had}(\omega)$ under Weyl operators to conclude that the first term on the right-hand side converges to $-(i / 2) E\left(\xi^{a} \nabla_{a} f, f\right) \varphi$ as $s \rightarrow 0$. A further application of the Weyl relations allows us to rewrite the second term in the form

$$
\begin{aligned}
s^{-1}\left[W\left(\tau_{s *} f-f\right)-\mathbf{1}\right] W(f) \varphi= & s^{-1} e^{-i s \nu} W(R(s))\left[W\left(-s \xi^{a} \nabla_{a} f\right)-\mathbf{1}\right] W(f) \varphi \\
& +s^{-1}\left(W\left(R(s) e^{-i s \nu}-\mathbf{1}\right) W(f) \varphi\right.
\end{aligned}
$$

where $\nu=E\left(R(s), \xi^{a} \nabla_{a} f\right) / 2$. Estimating the second term of Eq. (A.17), we see that

$$
\begin{aligned}
s^{-1}\left\|\left(W(R(s)) e^{-i s \nu}-\mathbf{1}\right) W(f) \varphi\right\| & \leq s^{-1}\|(W(R(s))-\mathbf{1}) W(f) \varphi\|+s^{-1}\left|e^{-i s \nu}-1\right|\|\varphi\| \\
& \leq\left\|\Phi\left(s^{-1} R(s)\right) W(f) \varphi\right\|+\frac{1}{2}\left|E\left(R(s), \xi^{a} \nabla_{a} f\right)\right|\|\varphi\|
\end{aligned}
$$

which tends to zero as $s \rightarrow 0$ by our two observations. The first term of Eq. (A.17) approaches a finite limit as $s \rightarrow 0$ :

$$
\begin{aligned}
\left\langle\psi, W(R(s)) s^{-1}\left[W\left(-s \xi^{a} \nabla_{a} f\right)\right.\right. & -\mathbf{1}] W(f) \varphi\rangle \\
& =\left\langle W(-R(s)) \psi, s^{-1}\left[W\left(-s \xi^{a} \nabla_{a} f\right)-\mathbf{1}\right] W(f) \varphi\right\rangle \\
& \rightarrow-i\left\langle\psi, \Phi\left(\xi^{a} \nabla_{a} f\right) W(f) \varphi\right\rangle
\end{aligned}
$$

so we have established that

$$
\left.\frac{d}{d s}\left\langle\psi, \alpha_{s} W(f) \varphi\right\rangle\right|_{s=0}=-i\left\langle\psi, \Phi\left(\xi^{a} \nabla_{a} f\right) W(f) \varphi\right\rangle-\frac{i}{2} E\left(\xi^{a} \nabla_{a} f, f\right)\langle\psi, \varphi\rangle,
$$

which can be put into the required form using (A.1), which is valid on $\operatorname{Had}(\omega)$.

The proof is completed by noting that $s \mapsto \alpha_{s} W(f) \eta=W\left(\tau_{s *} f\right) \eta$ and $s \mapsto \Phi\left(\xi^{a} \nabla_{a} \tau_{s *} f\right) \eta$ are continuous for $\eta \in \operatorname{Had}(\omega)$ (in the first case owing to our first observation and in the second because $\Phi(\cdot) \eta \in \mathcal{D}^{\prime}\left(M, \mathcal{H}_{\omega}\right)$ and $s \mapsto \xi^{a} \nabla_{a} \tau_{s *} f$ is continuous from $\left.\mathbb{R} \rightarrow \mathcal{D}(M)\right)$. Thus the right-hand side of (A.16) is continuous in $s$.

\section{A.2.2 The case $A \in \mathcal{A}^{\infty}$}

To extend Eq. (A.14) to the case $A \in \mathcal{A}^{\infty}$ it suffices (by Theorem A.1) to consider $A=\alpha_{f} W(h)$ for $f \in C_{0}^{\infty}(0, \infty), h \in \mathscr{D}(M ; \mathbb{R})$. Now

$$
\begin{aligned}
\left.\frac{1}{i} \frac{d}{d s} \ell\left(\alpha_{s} A\right)\right|_{s=0} & =-i \ell\left(\delta\left(\alpha_{f} W(h)\right)\right)=-i \ell\left(\alpha_{-\dot{f}} W(h)\right) \\
& =i \int d t \dot{f}(t) \ell\left(\alpha_{t} W(h)\right) .
\end{aligned}
$$

Owing to Prop. A.8, we may integrate by parts to find

$$
\left.\frac{1}{i} \frac{d}{d s} \ell\left(\alpha_{s} A\right)\right|_{s=0}=\frac{1}{i} \int d t f(t)\left(\gamma_{t_{0}}^{*} \ell\left(\left[: T:, \alpha_{t} W(h)\right]\right)\right)\left(g_{00}^{1 / 2}\right)
$$

using Eq. A.14 for $\alpha_{t} W(h)$. 
Our goal is now to show that

$$
\int d t f(t)\left(\gamma_{t_{0}}^{*} \ell\left(\left[: T:, \alpha_{t} W(h)\right]\right)\right)\left(g_{00}^{1 / 2}\right)=\left(\gamma_{t_{0}}^{*} \ell\left(\left[: T:, \alpha_{f} W(h)\right]\right)\right)\left(g_{00}^{1 / 2}\right) .
$$

To this end, we observe that

$$
\ell\left(\left[: T:, \alpha_{f} W(h)\right]\right)(F, G)=\int d t f(t) \ell\left(\left[: T:, \alpha_{t} W(h)\right]\right)(F, G), \quad F, G \in \mathscr{D}(M)
$$

in which the integrand is compactly supported and-by Prop. A.8 continuous. Accordingly, we may approximate the integral by Riemann sums. Defining

$$
T_{N}(F, G)=\frac{1}{N} \sum_{n \in \mathbb{Z}} f(n / N) \ell\left(\left[: T:, \alpha_{n / N} W(h)\right]\right)(F, G),
$$

only finitely many terms contribute for any given $N$, so $T_{N} \in \mathscr{D}^{\prime}(M \times M)$. Since the Riemann sums approximate the integral, we have $T_{N} \rightarrow T=\ell\left(\left[: T:, \alpha_{f} W(h)\right]\right)$ weakly, but we require the following stronger convergence property, whose proof is deferred to the end of this subsection.

Lemma A.9 $T_{N} \rightarrow T$ in $\mathscr{D}_{V}^{\prime}(M \times M)$, where $V=\left(\mathcal{N}^{+} \times \mathcal{Z}\right) \cup\left(\mathcal{Z} \times \mathcal{N}^{-}\right)$.

Now the conormal bundle of the map $\gamma_{t_{0}}$ is

$$
N_{\gamma_{t_{0}}}=\left\{(t, \underline{x}, \alpha, \underline{\xi} ; t, \underline{x}, \beta,-\underline{\xi}) \mid \alpha, \beta \in \mathbb{R},(\underline{x}, \underline{\xi}) \in T^{*} \Sigma\right\}
$$

and has trivial intersection with $V$. It therefore follows that $\gamma_{t_{0}}^{*} T_{N} \rightarrow \gamma_{t_{0}}^{*} T$ in $\mathscr{D}_{\gamma_{0} V}^{\prime * V}(\Sigma)$ and in particular that

$$
\left(\gamma_{t_{0}}^{*} T_{N}\right)\left(g_{00}^{1 / 2}\right) \rightarrow\left(\gamma_{t_{0}}^{*} T\right)\left(g_{00}^{1 / 2}\right)=\left(\gamma_{t_{0}}^{*} \ell\left(\left[: T:, \alpha_{f} W(h)\right]\right)\right)\left(g_{00}^{1 / 2}\right) .
$$

But we also have

$$
\begin{aligned}
\left(\gamma_{t_{0}}^{*} T_{N}\right)\left(g_{00}^{1 / 2}\right) & =\frac{1}{N} \sum_{n \in \mathbb{Z}} f(n / N)\left(\gamma_{t_{0}}^{*} \ell\left(\left[: T:, \alpha_{n / N} W(h)\right]\right)\left(g_{00}^{1 / 2}\right)\right. \\
& \longrightarrow \int d t f(t)\left(\gamma_{t_{0}}^{*} \ell\left(\left[: T:, \alpha_{t} W(h)\right]\right)\left(g_{00}^{1 / 2}\right)\right.
\end{aligned}
$$

since the final integrand is continuous. Thus (A.18) is established and the proof is complete.

Proof of Lemma A.9: It suffices to show that $U_{N} \rightarrow U$ in $\mathscr{D}_{V}^{\prime}(M \times M)$, where $U_{N}$ and $U$ are defined by analogy with $T_{N}$ and $T$, but with : $\Phi^{\otimes 2}$ : replacing : $T:$. It is clear that $U_{N} \rightarrow U$ weakly, so we need only show that $U_{N}$ converges in $\mathscr{D}_{V}^{\prime}(M \times M)$. Now

$\ell\left(\left[: \Phi^{\otimes 2}:, \alpha_{t} W(h)\right]\right)(F, G)=E\left(\tau_{t *} h, F\right)\left\langle\psi, W\left(\tau_{t *} h\right) \Phi(G) \varphi\right\rangle+E\left(\tau_{t *} h, G\right)\left\langle\psi, \Phi(F) W\left(\tau_{t *} h\right) \varphi\right\rangle$

and since the two terms each define a smooth distribution on $M \times M$, their contributions to $U_{N}$ may be treated separately. Accordingly, we let

$$
U_{N}^{(1)}(F, G)=\frac{1}{N} \sum_{n \in \mathbb{Z}} f(n / N) E\left(\tau_{n / N *} h, F\right)\left\langle\psi, W\left(\tau_{n / N *} h\right) \Phi(G) \varphi\right\rangle,
$$


and observe that

$$
\left|U_{N}^{(1)}(F, G)\right| \leq \frac{1}{N} \sum_{n \in \mathbb{Z}}|K(F)(n / N)|\|\Phi(G) \varphi\|,
$$

where $K(\cdot) \in \mathcal{D}^{\prime}\left(M, L^{1}(\mathbb{R})\right)$ was defined in Eq. (A.7). Since $t \mapsto K(F)(t)$ is smooth and compactly supported, we therefore have

$$
\left|U_{N}^{(1)}(F, G)\right| \leq 2\|K(F)\|_{L^{1}}\|\Phi(G) \varphi\|
$$

for all sufficiently large $N$. Putting this estimate together with the Hadamard condition, Lemma A.4 and Prop. 3.4, we conclude that $U_{N}^{(1)}$ converges in $\mathscr{D}_{Z \times \mathcal{N}^{-}}^{\prime}(M \times M)$. An analogous argument applied to

$$
U_{N}^{(2)}(F, G)=\frac{1}{N} \sum_{n \in \mathbb{Z}} f(n / N) E\left(\tau_{n / N *} h, G\right)\left\langle\psi, \Phi(F) W\left(\tau_{n / N *} h\right) \varphi\right\rangle,
$$

shows that $U_{N}^{(2)}$ converges in $\mathscr{D}_{\mathcal{N}+\times \mathbb{Z}}^{\prime}(M \times M)$. Thus $U_{N}=U_{N}^{(1)}+U_{N}^{(2)}$ converges in $\mathscr{D}_{V}^{\prime}(M \times M)$ as required.

\section{A.2.3 The case $A \in \mathcal{U}_{\mathrm{alg}}^{\infty}$}

The last step in the proof of Prop. A.6 is to extend the result to show that the energy density generates the dynamics for operators in $\mathcal{U}_{\text {alg }}^{\infty}$. Here, it is enough to establish Eq. A.14 with $A$ replaced by an operator of the form $e^{i A}$ for $A=A^{*} \in \mathcal{A}^{\infty}$, which may be arbitrarily well approximated in the graph norm of $\delta$ by the sequence

$$
S_{N}=\sum_{n=0}^{N} \frac{i^{n}}{n !} A^{n}
$$

of partial sums. Since $S_{N} \in \mathcal{A}^{\infty}$, and

$$
\left.\left.\frac{1}{i} \frac{d}{d s} \ell\left(\alpha_{s} S_{N}\right)\right|_{s=0} \longrightarrow \frac{1}{i} \frac{d}{d s} \ell\left(\alpha_{s} e^{i A}\right)\right|_{s=0}
$$

it remains only to show that

$$
\left(\gamma_{t_{0}}^{*} \ell\left(\left[: T:, S_{N}\right]\right)\right)\left(g_{00}^{1 / 2}\right) \longrightarrow\left(\gamma_{t_{0}}^{*} \ell\left(\left[: T:, e^{i A}\right]\right)\right)\left(g_{00}^{1 / 2}\right) .
$$

Lemma A.10 Suppose $A \in \mathcal{A}^{\infty}$. Then

$$
\begin{aligned}
\mid \ell\left(A^{j}\left[: \Phi^{\otimes 2}:, A\right] A^{k}\right)(f, g) \leq & \|A\|^{j+k}[\|B(g)\|\|\Phi(\bar{f}) \psi\|+\|B(f)\|\|\Phi(g) \varphi\|] \\
& +(j+k)\|A\|^{j+k-1}\|B(f)\|\|B(g)\|
\end{aligned}
$$

for any $j, k \in \mathbb{N}_{0}$.

Proof: We have

$$
\ell\left(A^{j}\left[: \Phi^{\otimes 2}:, A\right] A^{k}\right)(f, g)=\left\langle\Phi(\bar{f}) A^{* j} \psi, B(g) A^{k} \varphi\right\rangle-\left\langle B(f)^{*} A^{* j} \psi, \Phi(g) A^{k} \varphi\right\rangle .
$$


Now

$$
\Phi(g) A^{k} \varphi=A^{k} \Phi(g) \varphi+\sum_{r=0}^{k-1} A^{r} B(g) A^{k-1-r} \varphi
$$

SO

$$
\left\|\Phi(g) A^{k} \varphi\right\| \leq\|A\|^{k}\|\Phi(g) \varphi\|+k\|A\|^{k-1}\|B(g)\| .
$$

Putting this together with the analogous estimate on $\left\|\Phi(\bar{f}) A^{* j} \psi\right\|$, the result is proved by Cauchy-Schwarz.

In consequence, and using

$$
\ell\left(\left[: \Phi^{\otimes 2}:, A^{n}\right]\right)=\sum_{j=0}^{n} \ell\left(A^{j}\left[: \Phi^{\otimes 2}:, A\right] A^{n-1-k}\right)
$$

we obtain

$$
\begin{aligned}
\left|\ell\left(\left[: \Phi^{\otimes 2}:, S_{N}\right]\right)(f, g)\right| \leq & C[\|B(g)\|\|\Phi(\bar{f}) \psi\|+\|B(f)\|\|\Phi(g) \varphi\|] \\
& +C^{\prime}\|B(f)\|\|B(g)\|
\end{aligned}
$$

for constants $C, C^{\prime}$ independent of $N, f$ and $g$. Applying the remark following Prop. 3.4. the Hadamard condition and the fact that $B(\cdot)$ has empty wave-front set, we see that $\ell\left(\left[: \Phi^{\otimes 2}:, S_{N}\right]\right) \rightarrow \ell\left(\left[: \Phi^{\otimes 2}:, e^{i A}\right]\right)$ in $\mathscr{D}_{V}^{\prime}(M \times M)$ as required. Proposition A.6] is thus proved.

\section{A.3 A variation on the Bochner-Schwartz Theorem}

Theorem A.11 Suppose $S \in \mathscr{D}^{\prime}(\mathbb{R})$ is of positive type with

$$
\mathrm{WF}(S) \subset\{(\tau, \zeta) \mid \zeta>0\}
$$

Then i) $S \in \mathscr{S}^{\prime}(\mathbb{R})$ and $\widehat{S}$ is a polynomially bounded positive measure; ii) $(-\infty, u]$ has finite (necessarily polynomially bounded) measure with respect to $\widehat{S}$; iii) if $f_{n}$ is any sequence of Schwartz test functions with $f_{n}(\zeta)$ monotonically increasing to $\chi_{(-\infty, u)}(\zeta)$ for each $\zeta \in \mathbb{R}$ then $\widehat{S}\left(f_{n}\right)$ is monotonically increasing and

$$
\lim _{n \rightarrow \infty} \widehat{S}\left(f_{n}\right)=\int_{(-\infty, u)} d \zeta \widehat{S}(\zeta)
$$

Proof: Part (i) is the usual Bochner-Schwartz theorem (Theorem IX.10 in [36]), while (iii) follows by the monotone convergence theorem if (ii) holds. It is enough to prove (ii) for $u=0$. To this end, let $f \in C^{\infty}(\mathbb{R})$ be nonnegative with supp $f \subset(-\infty, 1)$ and $f=1$ on $\mathbb{R}^{-}$. Decomposing $f$ as $f(\zeta)=\sum_{n=0}^{\infty} g(\zeta+n)$ where $g \in C_{0}^{\infty}(-1,1)$ is nonnegative, we claim that

$$
\int d \zeta \widehat{S}(\zeta) g(\zeta-\eta) \rightarrow 0
$$

rapidly as $\eta \rightarrow-\infty$. Thus

$$
\sum_{n=0}^{\infty} \int d \zeta \widehat{S}(\zeta) g(\zeta+n)<\infty
$$


and since each term in this series is positive, the monotone convergence theorem entails that $\int d \zeta \widehat{S}(\zeta) f(\zeta)<\infty$. Accordingly, $\int_{\mathbb{R}^{-}} d \zeta \widehat{S}(\zeta)<\infty$ and the result is proved.

It remains to prove our claim (A.22). Let $G(\zeta)=(\widehat{S} \star \widetilde{g})(\zeta)=\widehat{S}(g(\cdot-\zeta))$, where we have written $\widetilde{g}(\zeta)=g(-\zeta)$. Then $G$ is smooth and polynomially bounded, with polynomially bounded derivatives. Moreover, by (i), since $g$ is nonnegative, $G$ is also nonnegative and we may write

$$
G(\zeta)=\int d \eta \widehat{S}(\eta) g(\eta-\zeta)
$$

Using the convolution theorem,

$$
S\left(\chi \widetilde{g}^{\vee} e_{\eta}\right)=\int d \zeta G(\zeta) \widehat{\chi}(\eta-\zeta)
$$

for any $\chi \in \mathscr{D}$. Choose $\chi$ so that $\widehat{\chi}$ is nonnegative and $\widehat{\chi}(\zeta)>1$ for $|\zeta|<1$. Then for any $\epsilon \in(0,1)$ we have

$$
0 \leq \inf _{\zeta \in(\eta-\epsilon, \eta+\epsilon)} G(\zeta) \leq(2 \epsilon)^{-1} S\left(\chi \widetilde{g}^{\vee} e_{\eta}\right), \quad \eta \in \mathbb{R}
$$

and since $G$ has polynomially bounded first derivative, there exists $C>0$ and $r>0$ such that

$$
0 \leq G(\eta) \leq(2 \epsilon)^{-1} S\left(\chi \widetilde{g}^{\vee} e_{\eta}\right)+\epsilon C(1+|\eta|)^{r}, \quad \eta \in \mathbb{R}, 0<\epsilon<1 .
$$

In particular, taking $\epsilon=(1+|\eta|)^{-(N+r+1)}$ and using the hypothesis (A.20) on $\mathrm{WF}(S)$, it follows that $(1+|\eta|)^{N} G(\eta) \rightarrow 0$ as $\eta \rightarrow-\infty$ for each $N \geq 0$, thereby establishing our claim.

\section{A.4 Integrability of $Q(u, \cdot)$}

Proof of Proposition 4.6: Let $f_{n}$ be a sequence of Schwartz test functions such that $\widehat{f}_{n}$ is a sequence of nonnegative (Schwartz) functions monotonically increasing to $\chi_{(-\infty, u)}$. Then by Theorem A.11

$$
\Gamma_{\underline{x}}^{*} T_{0}\left(\overline{f_{n}} \star \widetilde{f}_{n}\right)=(2 \pi)^{-1} \widehat{\Gamma_{\underline{x}}^{*} T_{0}}\left(\left|\widehat{f}_{n}\right|^{2}\right) \rightarrow \pi Q(u, \underline{x})
$$

for each $\underline{x} \in \Sigma$. Choosing $g_{n} \in \mathscr{D}(\mathbb{R})$ such that $g_{n}-f_{n} \rightarrow 0$ in $\mathscr{S}(\mathbb{R})$ and using the positive type property of $\Gamma_{\underline{x}}^{*} T_{0}$, we have

$$
0 \leq \Gamma_{\underline{x}}^{*} T_{0}\left(\overline{g_{n}} \star \widetilde{g_{n}}\right) \rightarrow \pi Q(u, \underline{x}), \quad \underline{x} \in \Sigma .
$$

Lemma A.12 For each $h \in \mathscr{D}(\mathbb{R}), \Gamma_{\underline{x}}^{*} T_{0}(h) \in L^{1}(\Sigma, d \mu(\underline{x}))$ and

$$
\int d \mu(\underline{x}) \Gamma_{\underline{x}}^{*} T_{0}(h)=\mathfrak{T}(h),
$$

where

$$
\mathfrak{T}(h)=\Gamma^{*} T_{0}\left(h \otimes g_{00}^{-1 / 2}\right)
$$

and $\Gamma: M \rightarrow M \times M$ is given by $\Gamma(t, \underline{x})=(t, \underline{x} ; 0, \underline{x})$. The distribution $\mathfrak{T}$ is of positive type, with wave-front set contained in the right-hand side of (A.20). 
Applying the Lemma to $h=\overline{g_{n}} \star \widetilde{g_{n}}$, each $\Gamma_{\underline{x}}^{*} T_{0}\left(\overline{g_{n}} \star \widetilde{g_{n}}\right)$ is an $L^{1}(\Sigma, d \mu(\underline{x}))$ function with norm (= integral) converging to

$$
\lim _{n \rightarrow \infty} \int d \mu(\underline{x}) \Gamma_{\underline{x}}^{*} T_{0}\left(\overline{g_{n}} \star \widetilde{g_{n}}\right)=\lim _{n \rightarrow \infty} \mathfrak{T}\left(\overline{g_{n}} \star \widetilde{g_{n}}\right)=\lim _{n \rightarrow \infty} \mathfrak{T}\left(\overline{f_{n}} \star \widetilde{f_{n}}\right)=\int_{(-\infty, u)} d \zeta \widehat{\mathfrak{T}}(\zeta),
$$

where we have again used Theorem A.11, now applied to $\mathfrak{T}$. Putting this together with (A.29) and applying Fatou's lemma 35, we conclude that $Q(u, \cdot) \in L^{1}(\Sigma, d \mu)$ with

$$
0 \leq \int_{\Sigma} d \mu(\underline{x}) Q(u, \underline{x}) \leq \frac{1}{\pi} \int_{(-\infty, u)} d \zeta \widehat{\mathfrak{T}}(\zeta)
$$

the right-hand side of which is polynomially bounded by Theorem A.11. Thus $\mathfrak{Q}(u)=$ $\int_{\Sigma} d \mu(\underline{x}) Q(u, \underline{x})$ is polynomially bounded; monotonicity and left-continuity follow from the same properties of $Q(\cdot, \underline{x})$ and the monotone convergence theorem.

Proof of Lemma A.12: Note first that $\Gamma_{\underline{x}}^{*} T_{0}=\gamma_{\underline{x}}^{*} \Gamma^{*} T_{0}$, where $\gamma_{\underline{x}}: \mathbb{R} \rightarrow M$ is given by $\gamma_{\underline{x}}(t)=(t, \underline{x})$. Now, considering the definition of the distributional pull-back (cf. [25], Thms. 8.2.10, 8.2.12) we have

$$
\Gamma_{\underline{x}}^{*} T_{0}(h)=\gamma_{\underline{x}}^{*} \Gamma^{*} T_{0}(h)=\left[\left(h \otimes \delta_{\underline{x}}\right) \Gamma^{*} T_{0}\right]\left(1_{M}\right) .
$$

On the other hand, define $\left(\Gamma^{*} T_{0}\right)_{h} \in \mathscr{D}^{\prime}(\Sigma)$ by $\left(\Gamma^{*} T_{0}\right)_{h}(H)=\Gamma^{*} T_{0}(h \otimes H)$. Then $\mathrm{WF}\left(\left(\Gamma^{*} T_{0}\right)_{h}\right)=\emptyset$ and so

$$
\Gamma^{*} T_{0}\left(h \otimes g_{00}^{-1 / 2}\right)=\int_{\Sigma} d \mu(\underline{x})\left(\left(\Gamma^{*} T_{0}\right)_{h} \delta_{\underline{x}}\right)\left(1_{\Sigma}\right)
$$

[the $g_{00}^{-1 / 2}$ arises because the preferred densities on $\mathbb{R}, \Sigma$ and $M=\mathbb{R} \times \Sigma$ which identify distributions and distributional densities are related by $\left.\rho_{M}(t, \underline{x})=g_{00}(\underline{x})^{1 / 2} \rho_{\mathbb{R}}(t) \rho_{\Sigma}(\underline{x})\right]$. Finally, if $\delta_{n}$ is a sequence in $\mathscr{D}(\Sigma)$, converging to $\delta_{\underline{x}}$ in the Hörmander pseudo-topology on $\mathscr{D}_{\mathrm{WF}\left(\delta_{\underline{x}}\right)}^{\prime}(\Sigma)$ we may calculate

$$
\left(\left(\Gamma^{*} T_{0}\right)_{h} \delta_{\underline{x}}\right)\left(1_{\Sigma}\right)=\lim _{n \rightarrow \infty}\left(\Gamma^{*} T_{0}\right)_{h}\left(\delta_{n}\right)=\lim _{n \rightarrow \infty}\left(\left(h \otimes f_{n}\right) \Gamma^{*} T_{0}\right)\left(1_{M}\right)=\left[\left(h \otimes \delta_{\underline{x}}\right) \Gamma^{*} T_{0}\right]\left(1_{M}\right) .
$$

Putting this together with (A.34) and (A.35) we obtain (A.30).

It is clear from (A.30) that $\mathfrak{T}$ is of positive type, and a direct calculation of its wavefront set shows that $\mathrm{WF}(\mathfrak{T})$ is contained in the right-hand side of (A.20).

\section{A.5 Correlation functions for states on $\mathcal{A}$}

We begin our discussion of $n$-point correlation function for states on $\mathcal{A}$ with the following definitions:

Let $n \in \mathbb{N}$ and $F \in \mathscr{S}\left(\mathbb{R}^{n}\right)$, and define

$$
\mathbf{W}_{F}^{(\alpha)}\left(f_{1}, \ldots, f_{n}\right)=\int d t_{1} \cdots d t_{n} F\left(t_{1}, \ldots, t_{n}\right) \alpha_{t_{1}}\left(W\left(f_{1}\right)\right) \cdots \alpha_{t_{n}}\left(W\left(f_{n}\right)\right)
$$

for $f_{j} \in C_{0}^{\infty}(M, \mathbb{R})$, where we work in the defining representation $\pi_{\omega_{0}}$ of $\mathcal{A}$ with $W\left(f_{j}\right)=$ $\pi_{\omega_{0}}\left(\mathbf{W}\left(E f_{j}\right)\right)$; hence the expression on the right hand side exists as a weak integral in 
$\mathcal{B}\left(\mathcal{H}_{\omega_{0}}\right)$. Next we claim that the just defined objects are contained in $\mathcal{A}$. This can be seen by noting that there are sequences $h_{j}^{(k)} \in \mathscr{S}(\mathbb{R}), k \in \mathbb{N}$, so that

$$
\sum_{k=1}^{N} h_{1}^{(k)} \otimes \cdots \otimes h_{n}^{(k)} \underset{N \rightarrow \infty}{\longrightarrow} F \text { in } \mathscr{S}\left(\mathbb{R}^{n}\right) \text { and } \sum_{k=1}^{\infty}\left\|h_{1}^{(k)} \otimes \cdots \otimes h_{n}^{(k)}\right\|_{\infty}<\infty .
$$

This implies that $\alpha_{h_{1}^{(k)}} W\left(f_{1}\right) \cdots \alpha_{h_{n}^{(k)}} W\left(f_{n}\right)$ converges in norm to $\mathbf{W}_{F}^{(\alpha)}\left(f_{1}, \ldots, f_{n}\right)$ as $k \rightarrow$ $\infty$, and since clearly each $\alpha_{h_{j}^{(k)}} W\left(f_{j}\right)$ is in $\mathcal{A}, \mathbf{W}_{F}^{(\alpha)}\left(f_{1}, \ldots, f_{n}\right)$ is also contained in $\mathcal{A}$.

Now recall the definition of the $n$-point correlation functions of a state $\omega$ on the Weylalgebra $\mathfrak{A}[S, \sigma]^{11}$ : One says that $\omega$ is $C^{\infty}$-regular if, for each $n \in \mathbb{N}$, the map

$$
\mathbb{R}^{n} \times C_{0}^{\infty}(M, \mathbb{R})^{n} \ni\left(t_{1}, \ldots, t_{n} ; f_{1}, \ldots, f_{n}\right) \mapsto \omega\left(W\left(t_{1} f_{1}\right) \cdots W\left(t_{n} f_{n}\right)\right)
$$

is $C^{\infty}$ with respect to the $t_{j}$ and if the derivatives

$$
w_{n}^{(\omega)}\left(f_{1}, \ldots, f_{n}\right)=\left.(-i)^{n} \frac{\partial}{\partial t_{1}} \cdots \frac{\partial}{\partial t_{n}}\right|_{t_{j}=0} \omega\left(W\left(t_{1} f_{1}\right) \cdots W\left(t_{n} f_{n}\right)\right)
$$

induce, by requiring complex-linearity, distributions $w_{n}^{(\omega)} \in \mathscr{D}^{\prime}\left(M^{n}\right)$. These distributions are then called the $n$-point correlation functions of $\omega$. As is well-known (essentially by Wightman's reconstruction theorem, cf. [39]), a $C^{\infty}$-regular state $\omega$ induces a state $\bar{\omega}$ on the algebra $\mathscr{F}$ of abstract Klein-Gordon field operators. This algebra $\mathscr{F}$ is a $*$-algebra generated by a unit element 1 and a family of elements $\phi(f), f \in C_{0}^{\infty}(M)$, subject to the following relations:

(1) $f \mapsto \phi(f)$ is $\mathbb{C}$-linear,

(2) $\phi(f)^{*}=\phi(\bar{f})$,

(3) $\phi\left(\left(g^{a b} \nabla_{a} \nabla_{b}+m^{2}\right) f\right)=0$,

(4) $\left[\boldsymbol{\phi}\left(f_{1}\right), \boldsymbol{\phi}\left(f_{2}\right)\right]=i \sigma\left(E f_{1}, E f_{2}\right) \mathbf{1}$.

The state $\bar{\omega}$ is then defined on $\mathscr{F}$ by setting $\bar{\omega}(a \mathbf{1})=a(a \in \mathbb{C})$,

$$
\bar{\omega}\left(\phi\left(f_{1}\right) \cdots \phi\left(f_{n}\right)\right)=w_{n}^{(\omega)}\left(f_{1}, \ldots, f_{n}\right),
$$

and by requiring complex linearity. By the properties of the GNS-representation, the algebra $\mathscr{F}$ is $*$-isomorphic to the algebra of field operators $\Phi_{\omega}(f)=-\left.i \frac{d}{d t}\right|_{t=0} \pi_{\omega}(\mathrm{W}(t E f))$ by identifying $\phi(f)$ and $\Phi_{\omega}(f)$ (and by identifying unit operators).

Proceding along these lines, one can define an analogue of $n$-point correlation functions also for states $\omega$ on $\mathcal{A}$. We will say that a state $\omega$ on $\mathcal{A}$ is $C^{\infty}$-regular if for each $n \in \mathbb{N}$ and all $F \in \mathscr{S}\left(\mathbb{R}^{n}\right)$ the map

$$
\mathbb{R}^{n} \times C_{0}^{\infty}(M, \mathbb{R})^{n} \ni\left(t_{1}, \ldots, t_{n} ; f_{1}, \ldots, f_{n}\right) \mapsto \omega\left(\mathbf{W}_{F}^{(\alpha)}\left(t_{1} f_{1}, \ldots, t_{n} f_{n}\right)\right)
$$

\footnotetext{
${ }^{11}$ More precisely, $\omega$ is to be viewed here as a state on the represented Weyl-algebra $\pi_{\omega_{0}}(\mathfrak{A}[S, \sigma])$
} 
is $C^{\infty}$ with respect to the $t_{j}$ and if the derivatives

$$
\widetilde{w}_{n}^{(\omega)}\left(F ; f_{1}, \ldots, f_{n}\right)=\left.(-i)^{n} \frac{\partial}{\partial t_{1}} \cdots \frac{\partial}{\partial t_{n}}\right|_{t_{j}=0} \omega\left(\mathbf{W}_{F}^{(\alpha)}\left(t_{1} f_{1}, \ldots, t_{n} f_{n}\right)\right)
$$

induce distributions $\widetilde{w}_{n}^{(\omega)} \in\left(\mathscr{S}\left(\mathbb{R}^{n}\right) \otimes \mathscr{D}\left(M^{n}\right)\right)^{\prime}$.

Notice that there are many vector states with respect to the defining representation of $\mathcal{A}$ which are $C^{\infty}$-regular states on $\mathcal{A}$ : For example, all states on $\mathcal{A}$ induced by vectors $W(f) \Omega_{\omega_{0}}, f \in C_{0}^{\infty}(M, \mathbb{R})$, have this property.

We now proceed to establish the following result.

Theorem A.13 Let $\omega$ be a state on $\mathcal{A}$ which is $C^{\infty}$-regular. Let $F \in C_{0}^{\infty}\left(\mathbb{R}^{n}\right)$ with $\int d t_{1} \cdots d t_{n} F\left(t_{1}, \ldots, t_{n}\right)=1$ and define $F^{(\lambda)}\left(t_{1}, \ldots, t_{n}\right)=\lambda^{-n} F\left(t_{1} / \lambda, \ldots, t_{n} / \lambda\right)(\lambda>0)$ so that $F^{(\lambda)}$ approximates the $n$-dimensional Dirac-distribution as $\lambda \rightarrow 0$.

Then for all $n \in \mathbb{N}$ the limits

$$
\bar{w}_{n}^{(\omega)}\left(f_{1}, \ldots, f_{n}\right)=\lim _{\lambda \rightarrow 0} \widetilde{w}_{n}^{(\omega)}\left(F^{(\lambda)} ; f_{1}, \ldots, f_{n}\right), \quad f_{j} \in \mathscr{D}(M)
$$

exist, and induce distributions $\bar{w}_{n}^{(\omega)} \in \mathscr{D}^{\prime}\left(M^{n}\right)$ which will be called n-point correlation functions of $\omega$.

Moreover, the $\bar{w}_{n}^{(\omega)}, n \in \mathbb{N}$, induce a state $\bar{\omega}$ on the algebra $\mathscr{F}$ of field operators upon setting $\bar{\omega}(a \mathbf{1})=a(a \in \mathbb{C})$ and

$$
\bar{\omega}\left(\phi\left(f_{1}\right) \cdots \phi\left(f_{n}\right)\right)=\bar{w}_{n}^{(\omega)}\left(f_{1}, \ldots, f_{n}\right), \quad f_{j} \in \mathscr{D}(M),
$$

and by requiring linearity.

Proof. Let $\mathbf{s}=\left(s_{1}, \ldots, s_{n}\right) \in \mathbb{R}^{n}$ and define, for $F \in \mathscr{S}\left(\mathbb{R}^{n}\right)$,

$$
F_{\mathbf{s}}\left(t_{1}, \ldots, t_{n}\right)=F\left(t_{1}-s_{1}, \ldots, t_{n}-s_{n}\right) .
$$

Owing to the definition of $\mathbf{W}_{F}^{(\alpha)}\left(f_{1}, \ldots, f_{n}\right)$, it holds that

$$
\mathbf{W}_{F_{\mathbf{s}}}^{(\alpha)}\left(f_{1}, \ldots, f_{n}\right)=\mathbf{W}_{F}^{(\alpha)}\left(\tau_{s_{1} *} f_{1}, \ldots, \tau_{s_{n} *} f_{n}\right)
$$

and this implies

$$
\widetilde{w}_{n}^{(\omega)}\left(F_{\mathbf{s}} ; f_{1}, \ldots, f_{n}\right)=\widetilde{w}_{n}^{(\omega)}\left(F ; \tau_{s_{1} *} f_{1}, \ldots, \tau_{s_{n} *} f_{n}\right) .
$$

Let us now denote, for simplicity of notation, the distribution in $\left(\mathscr{S}\left(\mathbb{R}^{n}\right) \otimes \mathscr{D}\left(M^{n}\right)\right)^{\prime}$ induced by $\widetilde{w}_{n}^{(\omega)}$ simply by $w$. Then the last equation implies for this distribution the relation

$$
w((F * G) \otimes \varphi)=w(F \otimes(G \star \varphi)), \quad F \in \mathscr{S}\left(\mathbb{R}^{n}\right), \quad G \in \mathscr{D}\left(\mathbb{R}^{n}\right), \quad \varphi \in \mathscr{D}\left(M^{n}\right),
$$

where $F * G$ is the usual convolution of functions on $\mathbb{R}^{n}$ and

$$
(G \star \varphi)\left(t_{1}, \underline{x}_{1}, \ldots, t_{n}, \underline{x}_{n}\right)=\int d s_{1} \cdots d s_{n} G\left(s_{1}, \ldots, s_{n}\right) \varphi\left(t_{1}-s_{1}, \underline{x}_{1}, \ldots, t_{n}-s_{n}, \underline{x}_{n}\right) .
$$


Now we define

$$
\left(\Delta_{(1)} \varphi\right)\left(t_{1}, \underline{x}_{1}, \ldots, t_{n}, \underline{x}_{n}\right)=-\left(\partial_{t_{1}}^{2}+\cdots+\partial_{t_{n}}^{2}\right) \varphi\left(t_{1}, \underline{x}_{1}, \ldots, t_{n}, \underline{x}_{n}\right)
$$

and

$$
(\Delta G)\left(t_{1}, \ldots, t_{n}\right)=-\left(\partial_{t_{1}}^{2}+\cdots+\partial_{t_{n}}^{2}\right) G\left(t_{1}, \ldots, t_{n}\right) .
$$

Then relation (A.37) implies for all $F \in \mathscr{S}\left(\mathbb{R}^{n}\right), G \in \mathscr{D}\left(\mathbb{R}^{n}\right), \varphi \in \mathscr{D}\left(M^{n}\right)$ the following chain of equations:

$$
\begin{aligned}
w((F * G) \otimes \varphi) & =w\left((1-\Delta)^{-m} F *(1-\Delta)^{m} G \otimes \varphi\right) \\
& =w\left((1-\Delta)^{-m} F \otimes(1-\Delta)^{m} G \star \varphi\right) \\
& =w\left((1-\Delta)^{-m} F \otimes G \star\left(1-\Delta_{(1)}\right)^{m} \varphi\right) \\
& =w\left((1-\Delta)^{-m} F * G \otimes\left(1-\Delta_{(1)}\right)^{m} \varphi\right)
\end{aligned}
$$

which is valid for all $m \in \mathbb{N}$. Letting $G$ tend to the $n$-dimensional Dirac-distribution, we find

$$
w(F \otimes \varphi)=w\left((1-\Delta)^{-m} F \otimes\left(1-\Delta_{(1)}\right)^{m} \varphi\right)
$$

for all $F \in \mathscr{S}\left(\mathbb{R}^{n}\right), \varphi \in \mathscr{D}\left(M^{n}\right)$ and $m \in \mathbb{N}$. Hence, exploiting the regularizing property of $(1-\Delta)^{-m}$, one may choose $m$ so large that

$$
\lim _{\lambda \rightarrow 0} w\left(F^{(\lambda)} \otimes \varphi\right)=\lim _{\lambda \rightarrow 0} w\left((1-\Delta)^{-m} F^{(\lambda)} \otimes\left(1-\Delta_{(1)}\right)^{m} \varphi\right)
$$

exists (uniformly in $\varphi$ ) owing to the continuity of the functional $w$; this then implies that the resulting limit is a distribution in $\mathscr{D}^{\prime}\left(M^{n}\right)$ with respect to $\varphi$.

In order to show that the above definition of $\bar{\omega}$ really defines a state on $\mathscr{F}$, one needs to check first that $\bar{\omega}$ induces a linear functional on $\mathscr{F}$, i.e. that it respects the relations expressed in (1)-(4) above. Relation (1) is fulfilled since the $\bar{w}_{n}^{(\omega)}$ induce distributions (and are thus multilinear). The next relation (2) is essentially a consequence of $\left[(i t)^{-1}(W(t f)-\right.$ $\mathbf{1})]^{*}=(i / t)(W(-t f)-\mathbf{1})$ and so its proof is completely analogous to showing that the $n$-point correlation functions of a $C^{\infty}$-regular state on the Weyl-algebra induce a state on $\mathscr{F}$. Moreover, $\bar{w}_{n}^{(\omega)}\left(f_{1}, \ldots, f_{n}\right)=0$ if any of the $f_{j}$ is in the range of the Klein-Gordon operator since, in this case, $W\left(t_{j} f_{j}\right)=\mathbf{1}$, so that $\bar{\omega}$ respects relation (3). Finally, to check the CCR, note that

$$
\mathbf{W}_{F}^{(\alpha)}\left(f_{1}, \ldots, f_{j}, \ldots, f_{k}, \ldots, f_{n}\right)=\mathbf{W}_{F \cdot s_{j k}}^{(\alpha)}\left(f_{1}, \ldots, f_{k}, \ldots, f_{j}, \ldots, f_{n}\right)
$$

with $s_{j k}\left(t_{1}, \ldots, t_{n}\right)=\mathrm{e}^{i \sigma\left(E \tau_{t_{j} *} f_{j}, E \tau_{t_{k} *} f_{k}\right)}$. Inserting this into the definition of the $\widetilde{w}_{n}^{(\omega)}$ yields

$$
\begin{gathered}
\widetilde{w}_{n}^{(\omega)}\left(F ; f_{1}, \ldots, f_{j}, \ldots, f_{k}, \ldots, f_{n}\right)-\widetilde{w}_{n}^{(\omega)}\left(F ; f_{1}, \ldots, f_{k}, \ldots, f_{j}, \ldots, f_{n}\right) \\
=\widetilde{w}_{n-2}^{(\omega)}\left(G ; f_{1}, \ldots, f_{j}, \ldots, f_{k}, \ldots, f_{n}\right)
\end{gathered}
$$

where an $x$ over a symbol means that the corresponding entry doesn't appear, and the function $G$ is given by

$$
G\left(t_{1}, \ldots, \stackrel{\times}{t}_{j}, \ldots, \stackrel{x}{t}_{k}, \ldots, t_{n}\right)=\int d t_{j} d t_{k} F\left(t_{1}, \ldots, t_{n}\right) i \sigma\left(E \tau_{t_{j} *} f_{j}, E \tau_{t_{k} *} f_{k}\right) .
$$


These equations establish that $\bar{\omega}$ respects the CCR.

What remains to be checked is the positivity of $\omega$. To this end, let

$$
P=\left(a_{0} \mathbf{1}+\sum_{k=1}^{N} a_{k} \phi\left(f_{1}^{(k)}\right) \cdots \phi\left(f_{m_{k}}^{(k)}\right)\right)^{*}\left(a_{0} \mathbf{1}+\sum_{k=1}^{N} a_{k} \phi\left(f_{1}^{(k)}\right) \cdots \phi\left(f_{m_{k}}^{(k)}\right)\right)
$$

with $a_{0}, \ldots, a_{N} \in \mathbb{C}$ and $f_{j}^{(k)} \in C_{0}^{\infty}(M, \mathbb{R})$ be a generic positive element of $\mathscr{F}$. Then it holds that

$$
\begin{aligned}
\bar{\omega}(P)= & \left|a_{0}\right|^{2}+2 \operatorname{Re} a_{0} \sum_{k=1}^{N} \bar{w}_{m_{k}}^{(\omega)}\left(f_{1}^{(k)}, \ldots, f_{m_{k}}^{(k)}\right) \\
& +\sum_{k, \ell=1}^{N} \bar{a}_{k} a_{\ell} \bar{w}_{m_{k}+m_{\ell}}^{(\omega)}\left(f_{m_{k}}^{(k)}, \ldots, f_{1}^{(k)}, f_{1}^{(\ell)} \ldots, f_{m_{\ell}}^{(\ell)}\right) \\
= & \lim _{h \rightarrow \delta} \lim _{t \rightarrow 0} \omega\left(Q(h, t)^{*} Q(h, t)\right)
\end{aligned}
$$

where we define for $h \in C_{0}^{\infty}(\mathbb{R})$ with $\int d t h(t)=1$,

$$
Q(h, t)=a_{0} \mathbf{1}+\sum_{k=1}^{N} a_{k}\left(\frac{\alpha_{h} W\left(t f_{1}^{(k)}\right)-\mathbf{1}}{i t}\right) \cdots\left(\frac{\alpha_{h} W\left(t f_{m_{k}}^{(k)}\right)-\mathbf{1}}{i t}\right) .
$$

Hence the expressions over which the limits are taken are non-negative, and thus $\bar{\omega}(P) \geq 0$.

Acknowledgments We would like to thank Bernd Kuckert for discussions related to passivity, and for comments on the manuscript. We also thank him for having made his recent results available to us prior to publication. We also thank Muharrem Küskü for drawing our attention to some typographical errors in the preprint of this paper. The work of CJF was assisted by grant NUF-NAL/00075/G from the Nuffield Foundation and, in its later stages, by EPSRC grant GR/R25019/01.

\section{References}

[1] Bros, J., Buchholz, D., "Towards a relativistic KMS-condition", Nucl. Phys. B 429, 291 (1994)

[2] Brunetti, R., Fredenhagen, K., "Microlocal analysis and interacting quantum field theories: renormalization on physical backgrounds", Commun. Math. Phys. 208, 623 (2000)

[3] Brunetti, R., Fredenhagen, K., Köhler, M., "The microlocal spectrum condition and and Wick polynomials in curved spacetime", Commun. Math. Phys. 180, 633 (1996)

[4] Buchholz, D., Wichmann, E.H., "Causal independence and the energy-level density of states in local quantum field theory", Commun. Math. Phys. 106, 321 (1986) 
[5] Buchholz, D., Porrmann, M., "How small is the phase space in quantum field theory?", Ann. Inst. H. Poincaré 52, 237 (1990)

[6] Bratteli, O., Robinson, D.W., Operator algebras and quantum statistical mechanics, Vol. 1, 2nd edn, Springer-Verlag, Berlin-Heidelberg-New York, 1987

[7] Bratteli, O., Robinson, D.W., Operator algebras and quantum statistical mechanics, Vol. 2, 2nd edn, Springer-Verlag, Berlin-Heidelberg-New York, 1997

[8] D'Antoni, C., Hollands, S., "Nuclearity, local quasiequivalence and split property for Dirac quantum fields in curved spacetime", arXiv:math-ph/0106028

[9] Dimock, J., "Algebras of local observables on a manifold", Commun. Math. Phys. 77 (1980) 219

[10] Epstein, H., Glaser, V., Jaffe, A., "Nonpositivity of the energy density in quantized field theories", Nuovo Cimento 36, 1016 (1965)

[11] Fewster, C.J., Eveson, S.P., "Bounds on negative energy densities in flat spacetime", Phys. Rev. D 58, 084010 (1998)

[12] Fewster, C.J., "A general worldline quantum inequality", Class. Quantum Grav. 17, 1897 (2000)

[13] Fewster, C.J., Teo, E., "Bounds on negative energy densities in static spacetimes", Phys. Rev. D 59, 104016 (1999)

[14] Fewster, C.J., Teo, E., "Quantum inequalities and quantum interest as eigenvalue problems", Phys. Rev. D 61, 084012 (2000)

[15] Fewster, C.J., Verch, R., "A quantum weak energy inequality for Dirac fields in curved spacetime", Commun. Math. Phys. 225, 331 (2002)

[16] Flanagan, É.É., "Quantum inequalities in two-dimensional Minkowski spacetime", Phys. Rev. D 56, 4922 (1997)

[17] Ford, L.H., "Quantum coherence effects and the second law of thermodyamics", Proc. Roy. Soc. Lond. A364, 227 (1978)

[18] Ford, L.H., Roman, T.A., "Averaged energy conditions and quantum inequalities", Phys. Rev. D 51, 4277 (1995)

[19] Ford, L.H., Roman, T.A., "Restrictions on negative energy density in flat spacetime", Phys. Rev. D 55, 2082 (1997)

[20] Ford, L.H., Roman, T.A., "Quantum field theory constrains traversable wormhole geometries", Phys. Rev. D 53, 5496 (1996)

[21] Fulling, S.A., Narcowich, F.J., Wald, R.M., "Singularity structure of the two-point function in quantum field theory in curved spacetime, II", Ann. Phys. (N.Y.) 136, 243 (1981)

[22] Guido, D., Longo, R., "Natural energy bounds in quantum thermodynamics", Commun. Math. Phys. 218, 513 (2001) 
[23] Haag, R., Local quantum physics 2nd Ed., Springer Verlag, Berlin, 1996

[24] Haag, R., Swieca, J.A., "When does a quantum field theory describe particles?" Commun. Math. Phys. 1, 308 (1965)

[25] Hörmander, L., The analysis of linear partial differential operators I, Springer Verlag, Berlin, 1983

[26] Hörmander, L., "Fourier integral operators. I", Acta Math. 127, 79 (1971)

[27] Kay, B.S., Wald, R.M., "Theorems on the uniqueness and thermal properties of stationary, nonsingular, quasifree states on spacetimes with a bifurcate Killing Horizon", Phys. Rep. 207, 49 (1991)

[28] Kuckert, B., "Covariant thermodynamics of quantum systems: Passivity, semipassivity, and the Unruh effect", Ann. Phys. (N.Y.) 295216 (2002)

[29] Kuckert, B., " $\beta$-boundedness, semipassivity, and the KMS-condition", Commun. Math. Phys. 229, 369 (2002)

[30] Pfenning, M.J., "Quantum inequalities for the electromagnetic field", Phys. Rev. D 65, 024009 (2002)

[31] Pfenning, M.J., Ford, L.H., "The unphysical nature of 'warp drive'", Class. Quantum Grav. 14, $1743(1997)$

[32] Pfenning, M.J., Ford, L.H., "Scalar field quantum inequalities in static spacetimes", Phys. Rev. D 57, 3489 (1998)

[33] Pusz, W., Woronowicz, S.L., "Passive states and KMS states for general quantum systems", Commun. Math. Phys. 58 (1978) 273

[34] Radzikowski, M.J., "Micro-local approach to the Hadamard condition in quantum field theory in curved spacetime", Commun. Math. Phys. 179, 529 (1996)

[35] Reed, M., Simon, B., Methods of modern mathematical physics, Vol. 1, Academic Press, San Diego, 1975

[36] Reed, M., Simon, B., Methods of modern mathematical physics, Vol. 2, Academic Press, San Diego, 1975

[37] Sahlmann, H., Verch, R., "Passivity and microlocal spectrum condition", Commun. Math. Phys. 214, 705 (2000)

[38] Sahlmann, H., Verch, R., "Microlocal spectrum condition and Hadamard form for vectorvalued quantum fields in curved spacetime", Rev. Math. Phys. 13, 1203 (2001)

[39] Steater, R.F., Wightman, A.S., "PCT, spin and statistics, and all that", Benjamin, New York, 1964

[40] Strohmaier, A., Verch, R., Wollenberg, M., "Microlocal analysis of quantum fields on curved spacetimes: Analytic wavefront sets and Reeh-Schlieder theorems", J. Math. Phys. 43, 5514 (2002) 
[41] Summers, S.J., "Normal product states for fermions and twisted duality for CCR- and CAR-type algebras with applications to the Yukawa2 quantum field model", Commun. Math. Phys. 86, 111 (1982)

[42] Summers, S.J., "On the independence of local algebras in quantum field theory", Rev. Math. Phys. 2, 201 (1990)

[43] Verch, R., "Local definiteness, primarity and quasiequivalence of quasifree Hadamard quantum states in curved spacetime", Commun. Math. Phys. 160, 507 (1994)

[44] Verch, R., "Nuclearity, split property, and duality for the Klein-Gordon field in curved spacetime", Lett. Math. Phys. 29, 297 (1993)

[45] Verch, R., "Wavefront sets in algebraic quantum field theory", Commum. Math. Phys. 205 (1999) 337

[46] Visser, M., Barcelo, C., "Energy conditions and their cosmological implications", arXiv:gr-qc/0001099

[47] Vollick, D.N., "Quantum inequalities in curved two dimensional spacetime", Phys. Rev. D 61, $084022(2000)$

[48] Wald, R.M., Quantum field theory in curved spacetime and black hole thermodynamics, University of Chicago Press, Chicago, 1994

[49] Weinless, M., "Existence and uniqueness of the vacuum for linear quantized fields", J. Funct. Anal. 4, 350 (1969) 